Supporting Information

\title{
New Catalysts for Suzuki-Miyaura Coupling Reactions of Heteroatom-Substituted Heteroaryl Chlorides
}

\author{
Anil S. Guram,* Xiang Wang, Emilio E. Bunel, Margaret M. Faul, \\ Robert D. Larsen, and Michael J. Martinelli
}

Chemistry Research and Discovery, Amgen Inc., One Amgen Center Drive, Thousand Oaks, California 91320

aguram@amgen.com

\section{Experimental Section}

General. All experiments were performed under nitrogen atmosphere in screw-cap vials. All reagents including di-tert-butylphosphine, dicylclohexylphosphine, $\mathrm{Pd}_{2} \mathrm{dba}_{3}, \mathrm{PdCl}_{2}(\mathrm{COD})$, aryl- and heteroaryl halides, aryl- and heteroaryl boronic acids/esters, and organic/inorganic bases were purchased from commercial sources and used as received. Anhydrous, sure-seal grade organic solvents including toluene, tetrahydrofuran (THF), acetonitrile $\left(\mathrm{CH}_{3} \mathrm{CN}\right)$, dioxane, 1-butanol (1-BuOH), dimethoxyethane (DME), dimethylacetamide (DMAC), and pentane were used as purchased. Distilled water purged with nitrogen was used. Stock solutions of catalysts were generally used particularly for high TON experiments. Commercially available pre-packed silica gel plugs and hexanes/ethyl acetate solvents were used for column chromatographic purification of oragnic products. The syntheses of $\operatorname{PdCl}_{2}\left\{\mathrm{PR}_{2}\left(\mathrm{Ph}-\mathrm{R}^{\prime}\right)\right\}_{2}$ complexes were typically performed on $4.0-5.0 \mathrm{mmol}$ scale of the aryl halide. Few of the $\mathrm{PdCl}_{2}\left\{\mathrm{PR}_{2}(\mathrm{Ph}-\right.$ $\left.\left.\mathrm{R}^{\prime}\right)\right\}_{2}$ complexes are now commercially available. The Suzuki coupling reactions were typically performed on $1.0 \mathrm{mmol}$ scale of the aryl halide. The yields correspond to isolated products of $>95 \%$ purity as determined by NMR, GCMS and/or HPLC. The conversions were determined by GCMS area\% in the presence of a standard (BHT). The catalyst turnover numbers (TON) were determined in some cases using calibrated LC area\% (calibrated using purified product). NMR spectra were recorded on $400 \mathrm{MHz}$ instrument and all NMR $\left({ }^{1} \mathrm{H},{ }^{13} \mathrm{C},{ }^{31} \mathrm{P}\right.$, and $\left.{ }^{19} \mathrm{~F}\right)$ chemical shifts are reported in parts per million (ppm) and all coupling constants are reported in Hertz (Hz).

Syntheses of $\mathbf{P d C l}_{2}\left\{\mathbf{P R}_{\mathbf{2}}\left(\mathbf{P h}-\mathbf{R}^{\prime}\right)\right\}_{2}$ complexes $\left(\mathbf{A}: \mathrm{R}={ }^{t} \mathrm{Bu}, \mathrm{R}^{\prime}=p-\mathrm{CF}_{3} ; \mathbf{B}: \mathrm{R}={ }^{t} \mathrm{Bu}, \mathrm{R}{ }^{\prime}=\mathrm{H} ; \mathbf{B}^{\prime}: \mathrm{R}=\mathrm{Cy}\right.$, $\mathrm{R}^{\prime}=\mathrm{H} ; \mathbf{C}: \mathrm{R}={ }^{t} \mathrm{Bu}, \mathrm{R}^{\prime}=3,4,5$-tri-OMe; $\mathbf{D}: \mathrm{R}={ }^{t} \mathrm{Bu}, \mathrm{R}^{\prime}=p$-OMe; $\mathbf{E}: \mathrm{R}={ }^{t} \mathrm{Bu}, \mathrm{R}^{\prime}=p-\mathrm{NMe}_{2} ; \mathbf{E}^{\prime}: \mathrm{R}=\mathrm{Cy}$, 
$\left.\mathrm{R}^{\prime}=p-\mathrm{NMe}_{2}\right)$. The syntheses were typically performed on a 4.0 - 5.0 mmol scale of the aryl halide. A toluene or xylene solution of di-alkylphosphine (1.0 equiv), aryl bromide (1.0 equiv), $\mathrm{NaO}^{t} \mathrm{Bu}$ (1.5 equiv) and $\mathrm{Pd}_{2} \mathrm{dba}_{3}(1 \mathrm{~mol} \%)$ was stirred at $90-130{ }^{\circ} \mathrm{C}$ for $12 \mathrm{~h}$. The reaction was cooled to ambient temperature and filtered through a short silica gel plug. The silica gel plug was rinsed with toluene or dioxane. The combined filtrate was concentrated under vacuum and the residue was dissolved in THF. Alternatively, the combined filtrate was used as such. The $\mathrm{PdCl}_{2}(\mathrm{COD})$ (0.35 - 0.40 equiv) complex was added as a solid and the reaction mixture was stirred at ambient temperature for $12 \mathrm{~h}$. For complex B', a 2:1 reaction mixture of commercially available $\mathrm{PCy} 2 \mathrm{Ph}$ and $\mathrm{PdCl}_{2}(\mathrm{COD})$ in THF was stirred at ambient temperature for $12 \mathrm{~h}$. The reaction mixture was filtered, and the yellow solid was washed with pentane and dried under vacuum. The desired complexes were typically obtained in about 85\% isolated yields.

$\operatorname{PdCl}_{2}\left\{\mathbf{P}^{t} \mathbf{B u}_{2}\left(\mathbf{P h}-\boldsymbol{p}-\mathbf{C F}_{3}\right)\right\}_{2}$ (A): ${ }^{1} \mathbf{H}$ NMR $\left(\mathrm{CDCl}_{3}\right): \delta 8.00$ (br. s, 4H), 7.59 (br. d, J = 7.9, 4H), 1.62 (br. t, $\left.J_{P H}=7.0,36 \mathrm{H}\right) .{ }^{31} \mathbf{P} \mathbf{N M R}\left(\mathrm{CDCl}_{3}\right): \delta$ 55.7. ${ }^{19} \mathbf{F} \mathbf{N M R}\left(\mathrm{CDCl}_{3}\right): \delta$ - 63.4. Elemental Analysis: Calculated: C 47.54, H 5.85; Found: C 47.57, H 6.05. Raman: $\mathrm{cm}^{-1} 297.9$ (Pd-Cl).

$\mathbf{P d C l}_{2}\left\{\mathbf{P}^{t} \mathbf{B u}_{2}(\mathbf{P h})\right\}_{2}$ (B): ${ }^{1} \mathbf{H}$ NMR $\left(\mathrm{CD}_{2} \mathrm{Cl}_{2}\right): \delta 7.9-7.8(\mathrm{~m}, 4 \mathrm{H}), 7.36-7.30(\mathrm{~m}, 6 \mathrm{H}), 1.56$ (br. t, $J_{P H}=$ 6.9, 36H). ${ }^{31}$ P NMR $\left(\mathrm{CD}_{2} \mathrm{Cl}_{2}\right): \delta$ 54.4. Raman: $\mathrm{cm}^{-1} 300.9$ (Pd-Cl).

$\operatorname{PdCl}_{2}\left\{\mathbf{P C y}_{2}(\mathbf{P h})\right\}_{2}$ (B'): ${ }^{1} \mathbf{H}$ NMR $\left(\mathrm{C}_{6} \mathrm{D}_{6}\right): \delta 7.90(\mathrm{~m}, 4 \mathrm{H}), 7.29-7.18(\mathrm{~m}, 6 \mathrm{H}), 2.93-1.16(\mathrm{~m}, 44 \mathrm{H}) .{ }^{31} \mathbf{P}$ NMR $\left(\mathrm{C}_{6} \mathrm{D}_{6}\right): \delta 41.9$. Elemental Analysis: Calculated: C 59.55, H 7.50; Found: C 59.38, H 7.01.

$\operatorname{PdCl}_{2}\left\{\mathbf{P}^{t} \mathrm{Bu}_{2}(\mathbf{P h}-3,4,5 \text {-tri-OMe) }\}_{2}\right.$ (C): ${ }^{1} \mathbf{H}$ NMR $\left(\mathrm{C}_{6} \mathrm{D}_{6}\right): \delta 7.41$ (t, $\left.J_{P H}=4.7,4 \mathrm{H}\right), 3.93$ (s, $\left.6 \mathrm{H}\right), 3.71$ (s, 12H), 1.76 (br. t, $\left.J_{P H}=6.8,36 \mathrm{H}\right) .{ }^{31} \mathbf{P}$ NMR $\left(\mathrm{C}_{6} \mathrm{D}_{6}\right): \delta$ 69.4. Elemental Analysis: Calculated: C 50.91, H 7.29; Found: C 50.74, H 7.03.

$\mathbf{P d C l}_{2}\left\{\mathbf{P}^{t} \mathbf{B u}_{2}(\mathbf{P h}-\mathbf{p}-\mathbf{O M e})\right\}_{2}$ (D): Isolated material contained 1,4-dioxane (0.25 mol equiv). ${ }^{\mathbf{1}} \mathbf{H}$ NMR $\left(\mathrm{C}_{6} \mathrm{D}_{6}\right): \delta 8.06-8.02(\mathrm{p}, J=4.3,4 \mathrm{H}), 6.85(\mathrm{~d}, J=8.6,4 \mathrm{H}), 3.32(\mathrm{~s}, 6 \mathrm{H}) .1 .77$ (br. t, $\left.J=6.8,36 \mathrm{H}\right)$. Elemental Analysis: Calculated: C 53.28, H 7.45; Found: C 53.36, H 7.03. 
$\operatorname{PdCl}_{2}\left\{\mathbf{P}^{t} \mathbf{B u}_{2}\left(\mathbf{P h}-\boldsymbol{p}-\mathrm{NMe}_{2}\right)\right\}_{2}$ (E): ${ }^{1} \mathbf{H}$ NMR $\left(\mathrm{CDCl}_{3}\right): \delta 7.5$ (br. s, 4H), 6.42 (d, J = 8.3, 4H), 2.75 (s, $\left.12 \mathrm{H}\right)$, $1.38\left(\mathrm{t}, J_{P H}=6.6,36 \mathrm{H}\right) .{ }^{31} \mathbf{P}$ NMR $\left(\mathrm{CDCl}_{3}\right): \delta$ 52.1. Elemental Analysis: Calculated: C 54.28, H 7.97; Found: C 53.17, H 7.58. Raman: $\mathrm{cm}^{-1} 295.8$ (Pd-Cl).

$\operatorname{PdCl}_{2}\left\{\mathbf{P C y}_{2}\left(\mathbf{P h}-\mathbf{p}-\mathrm{NMe}_{2}\right)\right\}_{2}$ (E'): ${ }^{1} \mathbf{H}$ NMR $\left(\mathrm{C}_{6} \mathrm{D}_{6}\right): \delta 7.91(\mathrm{~m}, 4 \mathrm{H}), 6.64(\mathrm{~d}, J=8.8,4 \mathrm{H}), 3.03-1.28$ (m, 44H), 2.52 (s, 12H). ${ }^{31}$ P NMR $\left(\mathrm{C}_{6} \mathrm{D}_{6}\right): \delta$ 39.2. Elemental Analysis: Calculated: C 59.15, H 7.94; Found: C 58.85, H 7.63.

General procedure for the Suzuki-Miyaura coupling reactions. All Suzuki-Miyaura coupling reactions (Tables $1-9$, equations 1 and 2) were typically performed on a $1.0 \mathrm{mmol}$ scale of the aryl halide. A mixture of aryl halide (1.0 equiv), aryl boronic acid/ester (1.2 - 1.5 equiv), base (2.0 - 3.0 equiv.) and catalyst $(0.01-2.0 \mathrm{~mol} \%)$ in aqueous solvent ( 3-4 mL for $1.0 \mathrm{mmol}$ starting substrate, $\sim 10 \%$ water) was stirred at $80-100{ }^{\circ} \mathrm{C}$ in screw-capped glass vials for $5 \mathrm{~h}-20 \mathrm{~h}$. The reaction was cooled to ambient temperature and extracted in organic solvent (ether or dichloromethane). The organic extract was subjected to an aqueous work-up (when product stability permitted, $1 \mathrm{~N} \mathrm{NaOH}$ was used to facilitate removal of excess boronic acid), dried over anhydrous $\mathrm{Na}_{2} \mathrm{SO}_{4}$, and concentrated under vacuum. The crude material was purified by column chromatography on silica gel using hexanes and hexanes/ethyl acetate or $5 \%$ methanol in ethyl acetate as eluents. High TON experiments were stirred at $80-100{ }^{\circ} \mathrm{C}$ for $24 \mathrm{~h}$. Additional details are provided in the schematics and text.

2-(o-Tolyl)-3-pyridinamine (Table 5, entry 1). This compound was isolated as a white solid (93 \% yield) from the reaction of 2-chloropyridin-3-amine and $o$-tolylboronic acid using the general procedure. ${ }^{1} \mathbf{H}$ NMR $\left(\mathrm{CDCl}_{3}\right.$ ): $\delta 8.08$ (d, $\left.J=4.5,1 \mathrm{H}\right), 7.29-7.26$ (m, 4H), 7.08-7.00 (m, 2H), 3.59 (s br, 2H), 2.17 (s, 3H). ${ }^{13} \mathrm{C}$ NMR $\left(\mathrm{CDCl}_{3}\right): \delta$ 146.0, 140.3, 139.2, 137.4, 136.7, 130.7, 129.1, 128.5, 126.3, 123.2, 121.9, 19.3.

2-(p-Tolyl)-3-pyridinamine (Table 5, entry 2). This compound was isolated as a white solid (95 \% yield) from the reaction of 2-chloropyridin-3-amine and p-tolylboronic acid using the general procedure. ${ }^{1} \mathbf{H}$ NMR $\left(\mathrm{CDCl}_{3}\right): \delta 8.1(\mathrm{~d} / \mathrm{d}, J=4.1 / 1.8,1 \mathrm{H}), 7.55(\mathrm{~d}, J=8.2,2 \mathrm{H}), 7.26(\mathrm{~d}, J=7.8,2 \mathrm{H}), 7.04-6.98(\mathrm{~m}$, 
2H), 3.84 (s br, 2H), 2.39 (s, 3H). ${ }^{13} \mathbf{C}$ NMR $\left(\mathrm{CDCl}_{3}\right): \delta 145.1,140.0,139.9,138.0,135.7,129.4,128.3$, 122.8, 122.6, 21.3.

2-\{4-(Trifluoromethyl)phenyl\}-3-pyridinamine (Table 5, entry 3). This compound was isolated as a white solid (92 \% yield) from the reaction of 2-chloropyridin-3-amine and 4(trifluoromethyl)phenylboronic acid using the general procedure. ${ }^{1} \mathbf{H}$ NMR $\left(\mathrm{CDCl}_{3}\right): \delta 8.12(\mathrm{~d} / \mathrm{d}, J=$ 4.5/1.6, 1H), 7.81 (d, $J=8.0,2 \mathrm{H}), 7.71(\mathrm{~d}, J=8.3,2 \mathrm{H}), 7.10-7.02(\mathrm{~m}, 2 \mathrm{H}), 3.85$ (s br). ${ }^{13} \mathbf{C} \mathbf{~ N M R}$ $\left(\mathrm{CDCl}_{3}\right): \quad \delta 143.1,142.3,140.2,140.2,130.1\left(\mathrm{q},{ }^{2} J_{C F}=32.5\right), 128.9,125.7\left(\mathrm{q},{ }^{3} J_{C F}=3.5\right), 123.8,123.2$, $124.2\left(\mathrm{q},{ }^{1} J_{C F}=272\right)$.

2-(4-Methoxyphenyl)-3-pyridinamine (Table 5, entry 4). This compound was isolated as a white solid (93\% yield) from the reaction of 2-chloropyridin-3-amine and 4-methoxyphenylboronic acid using the general procedure. ${ }^{1} \mathbf{H}$ NMR $\left(\mathrm{CDCl}_{3}\right): \delta 8.08(\mathrm{~d} / \mathrm{d}, J=3.7 / 1.1,1 \mathrm{H}), 7.60(\mathrm{~d}, J=8.4,2 \mathrm{H}), 7.01-6.96(\mathrm{~m}$, 4H), 3.85 (s br, 2H, partially overlapped), 3.82 (s, 3H). ${ }^{13} \mathrm{C}$ NMR $\left(\mathrm{CDCl}_{3}\right): \delta 159.5,144.8,140.1,139.8$, 131.1, 129.8, 122.7, 122.6, 114.2, 55.4.

2-\{4-(Trifluoromethyl)phenyl\}-4-pyridinamine (Table 5, entry 5). This compound was isolated as a white solid (90 \% yield) from the reaction of 2-chloropyridin-4-amine and 4(trifluoromethyl)phenylboronic acid using the general procedure. ${ }^{1} \mathbf{H} \mathbf{N M R}\left(\mathrm{CDCl}_{3}\right): \delta 8.31$ (d, $J=5.4$, 1H), 8.0 (d, $J=8.2,2 \mathrm{H}$ ), 7.67 (d, $J=8.2,2 \mathrm{H}$ ), 6.93 (s, 1H), 6.51 (br d, $J=5.5,1 \mathrm{H}$ ), 4.39 (br s, 2H). ${ }^{13} \mathbf{C}$ NMR (CDCl $)$ ): $\delta 156.8,153.7,150.3,143.2,130.5\left(\mathrm{q},{ }^{2} J_{C F}=32.2\right), 127.2,125.5\left(\mathrm{q},{ }^{3} J_{C F}=3.5\right), 124.3$ (q, $\left.{ }^{1} J_{C F}=272\right), 109.0,106.9$.

2-(4-Fluorophenyl)-4-pyridinamine (Table 5, entry 6). This compound was isolated as a white solid (93 $\%$ yield) from the reaction of 2-chloropyridin-4-amine and 4-fluorophenylboronic acid using the general procedure. ${ }^{1} \mathbf{H}$ NMR $\left(\mathrm{CDCl}_{3}\right)$ : $\delta 7.46-7.42(\mathrm{~m}, 2 \mathrm{H}), 7.14-7.10(\mathrm{~m}, 2 \mathrm{H}), 6.97-6.92(\mathrm{~m}, 3 \mathrm{H})$, signal for $\mathrm{NH}_{2}$ protons not observed. ${ }^{13} \mathrm{C}$ NMR $\left(\mathrm{CDCl}_{3}\right): \delta 161.3$ (d, $\left.{ }^{1} J_{C F}=246.0\right), 142.3,129.6\left(\mathrm{~d},{ }^{3} J_{C F}=3.4\right)$, 127.0, 126.58, $126.54\left(\mathrm{~d},{ }^{3} J_{C F}=7.8\right), 123.73,122.1,114.76\left(\mathrm{~d},{ }^{2} J_{C F}=21.5\right)$. 
2-Mesityl-4-pyridinamine (Table 5, entry 7). This compound was isolated as a white solid (98 \% yield) from the reaction of 2-chloropyridin-4-amine and mesitylboronic acid using the general procedure. ${ }^{1} \mathbf{H}$ NMR $\left(\mathrm{CDCl}_{3}\right): \delta 8.02$ (d, $\left.J=5.5,1 \mathrm{H}\right), 6.75$ (s, 2H), 6.23 (d, $\left.J=5.4,1 \mathrm{H}\right), 6.20$ (s, 1H), 4.57 (s br, $\left.2 \mathrm{H}\right)$, 2.17 (s, 3H), 1.90 (s, 6H). ${ }^{13} \mathrm{C}$ NMR $\left(\mathrm{CDCl}_{3}\right): \delta$ 159.0, 152.7, 148.4, 137.1, 135.9, 134.5, 127.0, 109.1, 106.8, 20.0, 18.9 .

2-Mesityl-3-pyridinamine (Table 5, entry 8). This compound was isolated as a white solid (92 \% yield) from the reaction of 2-chloropyrdin-3-amine and mesitylboronic acid using the general procedure. ${ }^{1} \mathbf{H}$ NMR $\left(\mathrm{CDCl}_{3}\right): \delta 7.98(\mathrm{~d}, J=4.5,1 \mathrm{H}), 6.92(\mathrm{~d} / \mathrm{d}, J=8.0 / 5.0,1 \mathrm{H}), 6.84(\mathrm{~d}, J=8.0,1 \mathrm{H}), 6.82(\mathrm{~s}, 2 \mathrm{H}), 3.45$ (s br, 2H), 2.20 (s, 3H), 1.88 (s, 6H). ${ }^{13} \mathbf{C ~ N M R ~}\left(\mathrm{CDCl}_{3}\right): \delta 145.8,140.5,139.4,137.6,136.5,133.8,128.6$, 122.8, 121.3, 21.2, 19.4 .

3-Methoxy-6-(4-methoxyphenyl)pyridazine (Table 6, entry 1). This compound was isolated as a white solid (97 \% yield) from the reaction of 3-chloro-6-methoxypyridazine and 4-methoxyphenylboronic acid using the general procedure. ${ }^{1} \mathbf{H}$ NMR $\left(\mathrm{CDCl}_{3}\right): \delta 7.96$ (d, $\left.J=8.8,2 \mathrm{H}\right), 7.72(\mathrm{~d}, J=9.2,1 \mathrm{H}), 7.27-6.99$ (m, 3H), 4.17 (s, 3H), 3.86 (s, 3H). ${ }^{13} \mathbf{C ~ N M R ~}\left(\mathrm{CDCl}_{3}\right): \delta 164.0,160.8,154.9,128.8,127.8,126.6,117.7$, 114.4, 55.4, 54.8 .

4-(4-Methoxyphenyl)-2-methylthiopyrimidine (Table 6, entry 2). This compound was isolated as a white solid (95 \% yield) from the reaction of 4-chloro-2-(methylthio)pyrimidine and 4methoxyphenylboronic acid using the general procedure. ${ }^{1} \mathbf{H} \mathbf{N M R}\left(\mathrm{CDCl}_{3}\right): \delta 8.45(\mathrm{~d}, J=5.3,1 \mathrm{H}), 8.05$

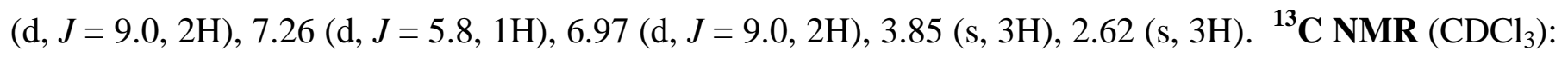
$\delta 172.5,163.3,162.2,157.4,128.8,128.7,114.3,111.0,55.5,14.2$.

4-\{2-(Methylthio)pyrimidin-4-yl\}benzonitrile (Table 6, entry 3). This compound was isolated as an offwhite solid (94\% yield) from the reaction of 4-chloro-2-(methylthio)pyrimidine and 4-cyanophenylboronic acid using the general procedure. ${ }^{1} \mathbf{H}$ NMR $\left(\mathrm{CDCl}_{3}\right): \delta 8.64(\mathrm{~d}, J=5.3,1 \mathrm{H}), 8.20(\mathrm{~d}, J=8.6,2 \mathrm{H}), 7.79(\mathrm{~d}$, $J=8.4,2 \mathrm{H}), 7.40(\mathrm{~d}, J=5.3,1 \mathrm{H}), 2.65(\mathrm{~s}, 3 \mathrm{H}) .{ }^{13} \mathbf{C} \mathbf{N M R}\left(\mathrm{CDCl}_{3}\right): \delta 173.5,161.8,158.3,140.5,132.7$, 127.8, 118.4, 114.5, 112.2, 14.3. 
Methyl 2-(4-Methoxyphenyl)-6-methylpyrimidine-4-carboxylate (Table 6, entry 4). This compound was isolated as an off-white solid (89 \% yield) from the reaction of methyl 2-chloro-6-methylpyrimidine-4carboxylate and 4-methoxyphenylboronic acid using the general procedure except (i) anhydrous toluene was used as solvent and (ii) 0.01 mol\% catalyst was used. ${ }^{1} \mathbf{H}$ NMR $\left(\mathrm{CDCl}_{3}\right): \delta 8.47(\mathrm{~d}, J=8.8,2 \mathrm{H}), 7.63$ (s, 1H), 6.98 (d, $J=8.6,2 \mathrm{H}), 4.02$ (s, 3H), 3.86 (s, 3H), 2.63 (s, 3H). ${ }^{13} \mathbf{C ~ N M R}\left(\mathrm{CDCl}_{3}\right): \delta$ 169.5, 165.5, 164.8, 162.1, 154.9, 130.2, 129.8, 117.3, 113.9, 55.4, 53.1, 24.6.

4-(2,6-Dimethylphenyl)-2-(methylthio)pyrimidine (Table 6, entry 5). This compound was isolated as a colorless oil (99\% yield) from the reaction of 4-chloro-2-(methylthio)pyrimidine and 2,6dimethylphenylboronic acid using the general procedure except 0.1 mol\% catalyst was used. ${ }^{1} \mathbf{H}$ NMR $\left(\mathrm{CDCl}_{3}\right): \delta 8.47(\mathrm{~d}, J=4.9,1 \mathrm{H}), 7.12(\mathrm{t}, J=7.6,1 \mathrm{H}), 7.01(\mathrm{~d}, J=7.5,2 \mathrm{H}), 6.80(\mathrm{~d}, J=5.0,1 \mathrm{H}), 2.47$ (s, 3H), 2.01 (s, 6H). ${ }^{13} \mathrm{C}$ NMR $\left(\mathrm{CDCl}_{3}\right): \delta 171.9,166.8,156.1,136.9,134.2,127.6,126.8,116.1,19.2,13.1$.

2,4-Dimethoxy-6-(4-methoxyphenyl)pyrimidine (Table 6, entry 6). This compound was isolated as an off-white solid (98\% yield) from the reaction of 6-chloro-2,4-dimethoxypyrimidine and 4methoxyphenylboronic acid using the general procedure except $0.1 \mathrm{~mol} \%$ catalyst was used. ${ }^{\mathbf{1}} \mathbf{H}$ NMR $\left(\mathrm{CDCl}_{3}\right): \delta 8.02$ (d, $\left.J=8.6,2 \mathrm{H}\right), 6.97$ (d, $\left.J=8.6,2 \mathrm{H}\right), 6.7$ (s, 1H), 4.07 (s, 3H), 3.99 (s, 3H), 3.86 (s, $\left.3 \mathrm{H}\right)$.

${ }^{13} \mathrm{C}$ NMR $\left(\mathrm{CDCl}_{3}\right): \delta 172.5,165.6,165.4,161.7,129.3,128.6,114.0,95.9,55.4,54.7,53.9$.

5-(6-Methoxypyridin-3-yl)pyridin-2-amine (Table 7, entry 1). This compound was isolated as a white solid (97\% yield) from the reaction of 5-chloropyridin-2-amine and 6-methoxypyridin-3-ylboronic acid using the general procedure. ${ }^{1} \mathbf{H}$ NMR $\left(\mathrm{CDCl}_{3}\right): \delta 8.27(\mathrm{~d} / \mathrm{d}, J=8 / 2.3,2 \mathrm{H}), 7.70(\mathrm{~d} / \mathrm{d}, J=8.6 / 2.6,1 \mathrm{H})$, 7.59 (d/d, $J=8.6 / 2.5,1 \mathrm{H}), 6.80$ (d, J = 8.6, 1H), 6.58 (d, $J=8.4,1 \mathrm{H}), 4.53$ (br s, $2 \mathrm{H}), 3.97$ (s, $3 \mathrm{H}) .{ }^{13} \mathrm{C}$ NMR $\left(\mathrm{CDCl}_{3}\right): \delta 163.4,157.7,146.1,144.2,136.8,136.2,127.4,124.2,110.9,108.6,53.5$.

4-(Pyridin-3-yl)pyridin-2-amine (Table 7, entry 2). This compound was isolated as an off-white solid (95 $\%$ yield) from the reaction of 4-chloropyridin-2-amine and pyridin-3-ylboronic acid using the general 
procedure. ${ }^{1}$ H NMR $\left(\mathrm{CDCl}_{3}\right): \delta 8.84(\mathrm{~s}, 1 \mathrm{H}), 8.65(\mathrm{~d}, J=3.5 \mathrm{~Hz}, 1 \mathrm{H}), 8.17$ (d, $\left.J=5.0,1 \mathrm{H}\right), 7.87(\mathrm{~d}, J=$ 7.5, 1H), $7.34-7.45(\mathrm{~m}, 1 \mathrm{H}), 6.86(\mathrm{~d}, J=5.0,1 \mathrm{H}), 6.69$ (s, $1 \mathrm{H}), 4.68$ (br. s, 2H). ${ }^{13} \mathbf{C}$ NMR $\left(\mathrm{CDCl}_{3}\right): \delta$ 158.3, 149.1, 148.2, 147.3, 146.3, 133.6, 133.4, 122.9, 111.6, 105.4.

2-(Pyridin-3-yl)pyridin-3-amine (Table 7, entry 3). This compound was isolated as a white solid (88 \% yield) from the reaction of chloropyridin-3-amine and pyridin-3-ylboronic acid using the general procedure. ${ }^{1} \mathbf{H}$ NMR $\left(\mathrm{CDCl}_{3}\right): \delta 8.96$ (s, $\left.1 \mathrm{H}\right), 8.63$ (d, $\left.J=4.0,1 \mathrm{H}\right), 8.15$ (d, $\left.J=3.5,1 \mathrm{H}\right), 8.03$ (d, $J=7.5$, 1H), 7.40-7.42 (m, 1H), 7.05 - 7.17 (m, 2H), 3.79 (s, br, 2H). ${ }^{13}$ C NMR $\left(\mathrm{CDCl}_{3}\right)$ : $\delta$ 147.2, 146.9, 139.0, 138.1, 138.1, 133.9, 132.2, 121.4, 121.3, 120.8.

4-(2-Fluoropyridin-3-yl)-2,6-dimethoxypyrimidine (Table 7, entry 4). This compound was isolated as an off-white solid (97\% yield) from the reaction of Cl-chloro-2,4-dimethoxypyrimidine and 2fluoropyridin-3-ylboronic acid using the general procedure. ${ }^{1} \mathbf{H}$ NMR $\left(\mathrm{CDCl}_{3}\right): \delta 8.92-8.99(\mathrm{~m}, 1 \mathrm{H}), 8.53$ (d, $J=5.0,1 \mathrm{H}), 7.60-7.57(\mathrm{~m}, 1 \mathrm{H}), 7.02(\mathrm{~s}, 1 \mathrm{H}), 4.31$ (s, 3H), 4.26 (s, 3H). ${ }^{13} \mathrm{C}$ NMR $\left(\mathrm{CDCl}_{3}\right): \delta$ 172.7, $165.2,161.2\left(\mathrm{~d}, J_{C F}=244\right), 159.1$ (d, $\left.J_{C F}=7.8\right), 148.8$ (d, $\left.J_{C F}=15.6\right), 141.2,121.9$ (d, $\left.J_{C F}=4.3\right), 119.8$ (d, $\left.J_{C F}=25.2\right), 101.7\left(\mathrm{~d}, J_{C F}=13.9\right), 54.8,54.1$.

4-(2-Fluoropyridin-3-yl)-2-(methylthio)pyrimidine (Table 7, entry 5). This compound was isolated as a white solid (92\% yield) from the reaction of 4-chloro-2-(methylthio)pyrimidine and 2-fluoropyridin-3ylboronic acid using the general procedure. ${ }^{1} \mathbf{H}$ NMR $\left(\mathrm{CDCl}_{3}\right): \delta 8.71(\mathrm{t}, J=8.8,1 \mathrm{H}), 8.61(\mathrm{~d}, J=5.5,1 \mathrm{H})$, $8.33(\mathrm{~s}, 1 \mathrm{H}), 7.56(\mathrm{~d}, J=3.5,1 \mathrm{H}), 7.33-7.42(\mathrm{~m}, 1 \mathrm{H}), 2.62(\mathrm{~s}, 3 \mathrm{H}) .{ }^{13} \mathbf{C} \mathbf{N M R}\left(\mathrm{CDCl}_{3}\right): \delta 172.3,161.2(\mathrm{~d}$, $J_{C F}=243$ ), 158.1, 158.0 (d, $\left.J_{C F}=7.8\right), 149.5$ (d, $J_{C F}=15.6$ ), 141.2, 122.1, 119.4 (d, $\left.J_{C F}=26.0\right), 115.5$ (d, $\left.J_{C F}=13.0\right), 14.2$.

3-(2-Fluoropyridin-3-yl)-6-methoxypyridazine (Table 7, entry 6). This compound was isolated as a white solid (99\% yield) from the reaction of 3-chloro-6-methoxypyridazine and 2-fluoropyridin-3ylboronic acid using the general procedure. ${ }^{1} \mathbf{H}$ NMR $\left(\mathrm{CDCl}_{3}\right): \delta 8.60-8.70(\mathrm{~m}, 1 \mathrm{H}), 8.31(\mathrm{~d}, J=4.5,1 \mathrm{H})$, 7.97 (d/d, $J=9.5 / 2.0,1 \mathrm{H}), 7.35-7.45(\mathrm{~m}, 1 \mathrm{H}), 7.10$ (d, $J=9.0,1 \mathrm{H}), 4.21$ (s, 3H). ${ }^{13} \mathbf{C}$ NMR $\left(\mathrm{CDCl}_{3}\right)$ : 
$\delta 164.4,160.5\left(\mathrm{~d}, J_{C F}=240\right), 150.4\left(\mathrm{~d}, J_{C F}=6.1\right), 148.4\left(\mathrm{~d}, J_{C F}=15.6\right), 140.9,130.0\left(\mathrm{~d}, J_{C F}=11.3\right)$, 122.3, $119.4\left(\mathrm{~d}, J_{C F}=26.8\right), 117.3,55.0$.

2-(p-Tolyl)thiophene (Table 8, entry 1). This compound was isolated as a white solid (96 \% yield) from the reaction of 2-chlorothiophene and $p$-tolylboronic acid using the general procedure. ${ }^{1} \mathbf{H} \mathbf{N M R}\left(\mathrm{CDCl}_{3}\right)$ : $\delta 7.48$ (d, $J=8.2,2 \mathrm{H}), 7.24$ (d, $J=3.5,1 \mathrm{H}), 7.2$ (d, $J=5,1 \mathrm{H}$ ), 7.15 (d, $J=7.9,2 \mathrm{H}$ ), 7.03 (d/d, $J=5.1 / 3.6$, 1H), 2.33 (s, 3H). ${ }^{13} \mathrm{C}$ NMR $\left(\mathrm{CDCl}_{3}\right): \delta 144.6,137.3,131.6,129.5,127.9,125.8,124.2,122.6,21.2$.

4-(4-Methoxyphenyl)-1,3,5-trimethyl-1H-pyrazole (Table 8, entry 2). This compound was isolated as a white solid (93 \% yield) from the reaction of 4-bromo-1,3,5-trimethylpyrazole and 4methoxyphenylboronic acid using the general procedure. ${ }^{1} \mathbf{H}$ NMR $\left(\mathrm{CDCl}_{3}\right): \delta 7.15$ (d, $\left.J=8.6,2 \mathrm{H}\right), 6.94$ $(J=8.6,2 \mathrm{H}), 3.82$ (s, 3H), 3.76 (s, 3H), 2.22 (s, 3H), 2.20 (s, 3H). ${ }^{13} \mathrm{C}$ NMR $\left(\mathrm{CDCl}_{3}\right): \delta$ 158.1, 145.0, 136.0, 130.5, 126.6, 118.8, 113.9, 55.3, 36.0, 12.4, 10.2.

4-(4-Fluorophenyl)-1,3,5-trimethyl-1H-pyrazole (Table 8, entry 3). This compound was isolated as a white solid (95 \% yield) from the reaction of 4-bromo-1,3,5-trimethylpyrazole and 4-fluorophenylboronic acid using the general procedure. ${ }^{1} \mathbf{H}$ NMR $\left(\mathrm{CDCl}_{3}\right): \delta 7.10(\mathrm{~d} / \mathrm{d} . J=8.8 / 5.7,2 \mathrm{H}), 6.99(\mathrm{t}, J=8.8,2 \mathrm{H})$, 3.69 (s, 3H), 2.13 (s, 3H), 2.12 (s, 3H). ${ }^{13}$ C NMR $\left(\mathrm{CDCl}_{3}\right): \delta 160.5$ (d, $\left.J=244\right), 143.9,135.1,129.9$ (d, $J$ $=7.7), 129.2(\mathrm{~d}, J=2.6), 117.3,114.2(\mathrm{~d}, J=20.7), 35.0,11.3,9.1 .{ }^{19} \mathbf{F} \mathbf{N M R}\left(\mathrm{CDCl}_{3}\right): \delta-116$.

3-(1,3,5-Trimethyl-1H-pyrazol-4-yl)pyridine (Table 8, entry 4). This compound was isolated as an offwhite solid (95\% yield) from the reaction of 4-bromo-1,3,5-trimethylpyrazole and pyridine-3-ylboronic acid using the general procedure. ${ }^{1} \mathbf{H}$ NMR $\left(\mathrm{CDCl}_{3}\right): \delta 8.50-8.52(\mathrm{~m}, 2 \mathrm{H}), 7.57(\mathrm{~d}, J=8.0,1 \mathrm{H}), 7.33(\mathrm{~d} / \mathrm{d}, J$ $=7.5,5.0,1 \mathrm{H}), 3.80$ (s, 3H), 2.25 (s, $6 \mathrm{H}) .{ }^{13} \mathrm{C}$ NMR $\left(\mathrm{CDCl}_{3}\right): \delta 150.3,147.3,145.2,136.7,136.5,130.2$, 123.4, 115.6, 36.1, 12.4, 10.2.

2-(6-Methoxypyridin-3-yl)benzo[d]oxazole (Table 8, entry 5). This compound was isolated as an offwhite solid (88 \% yield) from the reaction of 2-chlorobenzoxazole and 6-methoxypyridin-3-ylboronic acid 
using the general synthetic procedure with a modified work-up procedure where the product was precipitated by adding water to the reaction mixture in dioxane. The precipitated product was washed with water $(5 \mathrm{~mL})$, cold pentane $(1 \mathrm{~mL})$, and dired under vacuum. ${ }^{1} \mathbf{H}$ NMR $\left(\mathrm{CDCl}_{3}\right)$ : $\delta 9.04$ (br. s, $\left.1 \mathrm{H}\right), 8.36$ (br. d, $J=11.0,1 \mathrm{H}), 7.75$ (br. s, 1H), 7.56 (br. s, 1H), 7.34 (m, 2H), 6.88 (d, J = 8.8, 1H), 4.03 (s, $3 \mathrm{H}) .{ }^{13} \mathrm{C}$ NMR $\left(\mathrm{CDCl}_{3}\right): \delta 166.0,161.4,150.6,147.2,142.0,137.5,125.0,124.7,119.8,117.1,111.4,110.5,54.0$.

2-(Thiophen-2-yl)benzo[d]oxazole (Table 8, entry 6). This compound was isolated as a white solid (95 \% yield) from the reaction of 2-chlorobenzooxazole and 2-thiopheneboronic acid using the general procedure. ${ }^{1} \mathbf{H}$ NMR $\left(\mathrm{CDCl}_{3}\right): \delta 7.89(\mathrm{~d}, J=4.0,1 \mathrm{H}), 7.67$ - $7.78(\mathrm{~m}, 1 \mathrm{H}), 7.49$ - $7.58(\mathrm{~m}, 2 \mathrm{H}), 7.28$ - 7.37 (m, $\left.2 \mathrm{H}\right)$, 7.11 - $7.21(\mathrm{~m}, 1 \mathrm{H}) .{ }^{13} \mathrm{C}$ NMR $\left(\mathrm{CDCl}_{3}\right): \delta$ 159.1, 150.4, 142.0, 130.3, 130.0, 129.7, 128.3, 125.1, 124.8, 119.8, 110.5 .

2-(p-Tolyl)-3-pyridinamine (Equation 1). This compound was isolated as a white solid (96 \% yield) from the reaction of 2-chloro-3-pyridinamine and 4,4,5,5-tetramethyl-2-p-tolyl-1,3,2-dioxaborolane using the general procedure. Isolated material contained some 2,3-dimethylbutane-2,3-diol. The ${ }^{1} \mathrm{H}$ and ${ }^{13} \mathrm{C} N M R$ data matched entry 2, Table 5.

2-(Quinolin-6-yl)pyridine-4-amine (Equation 2). This compound was isolated as a white solid (92 \% yield) from the reaction of 2-chloro-4-pyridinamine and 6-(4,4,5,5-tetramethyl-1,3,2-dioxaborolan-2yl)quinoline using the general procedure. ${ }^{1} \mathbf{H}$ NMR $\left(\mathrm{CD}_{3} \mathrm{OD}\right): \delta 8.88(\mathrm{~d}, J=4.0,1 \mathrm{H}), 8.45(\mathrm{~d}, J=8.0$, 1H), 8.35 (s, $1 \mathrm{H}), 8.19$ (d, 8.6, 1H), 8.12 (d, 6.1, 2H), 7.60 - 7.57 (dd, J = 8.2, 4.2, 1H), 7.14 (s, $1 \mathrm{H}), 6.66$ $(\mathrm{d}, J=5.5,1 \mathrm{H}),-\mathrm{NH}_{2}$ protons not observed. ${ }^{13} \mathbf{C} \mathbf{N M R}\left(\mathrm{CDCl}_{3}\right): \delta 157.1,155.5,150.6,147.5,147.2$, 137.6, 136.9, 128.5, 128.4, 128.3, 126.4, 121.8, 108.0, 107.0. 

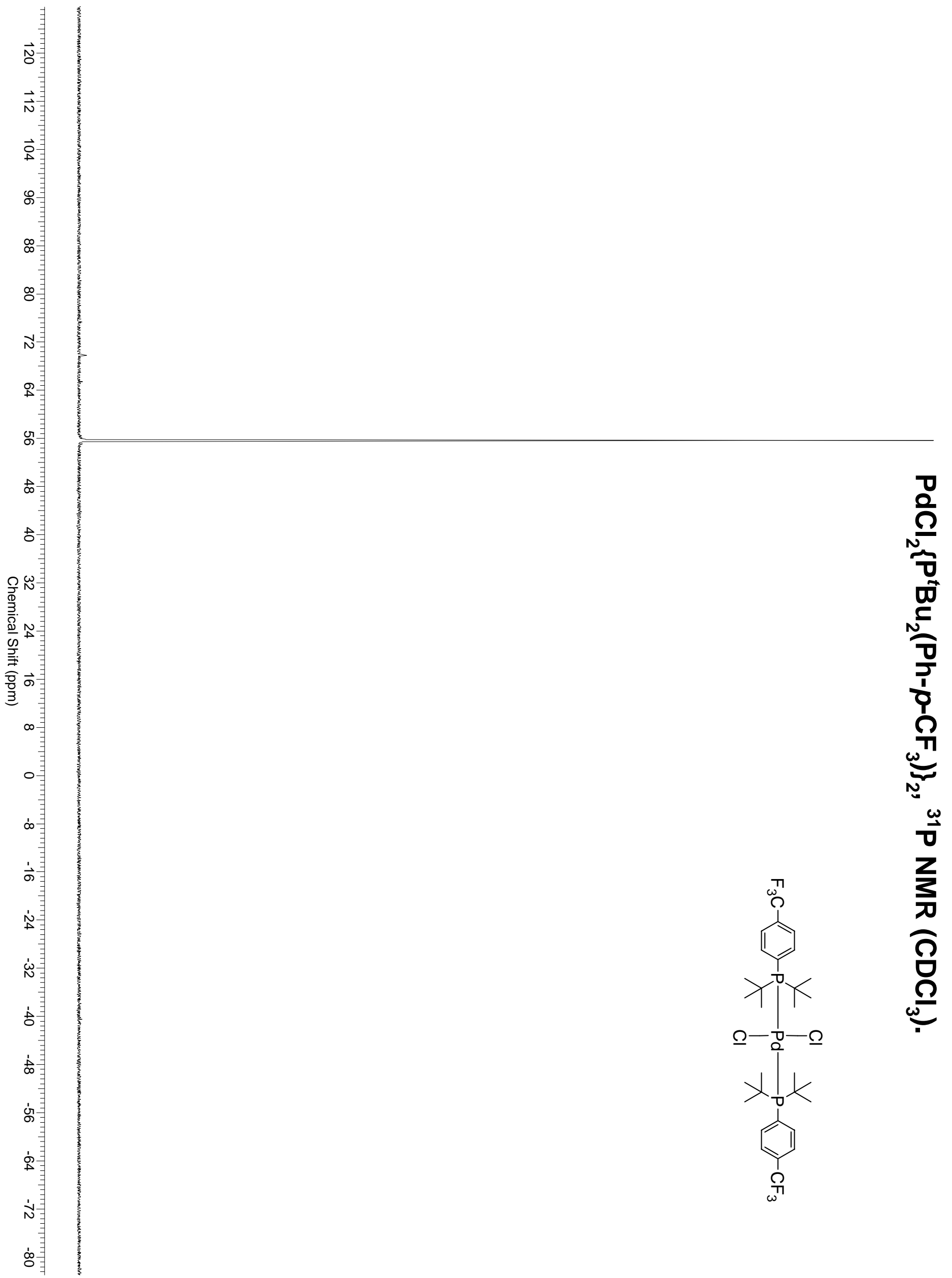

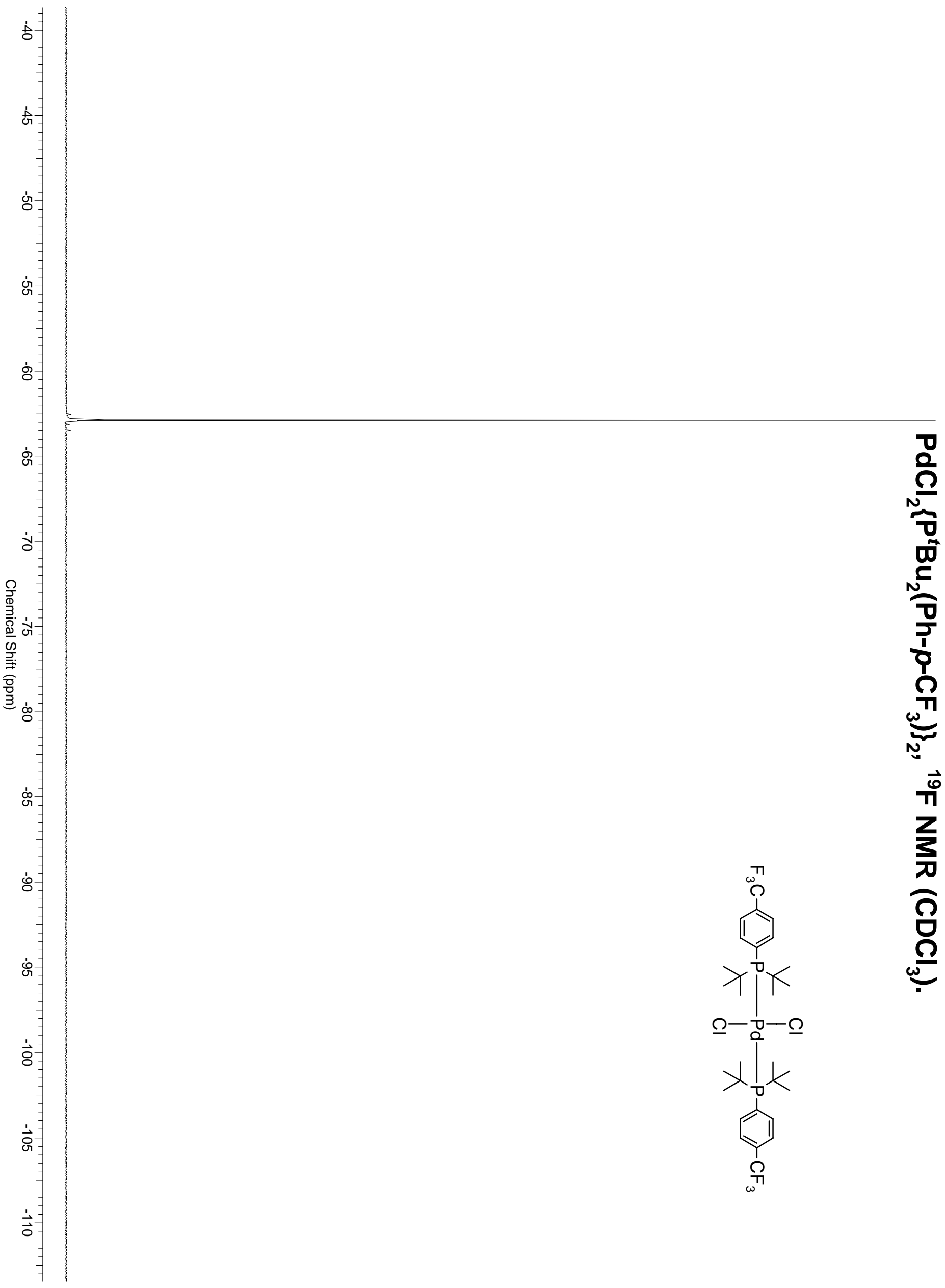

)
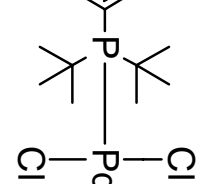

セ

㝋

ก

నِ

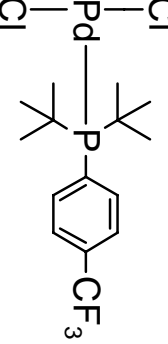




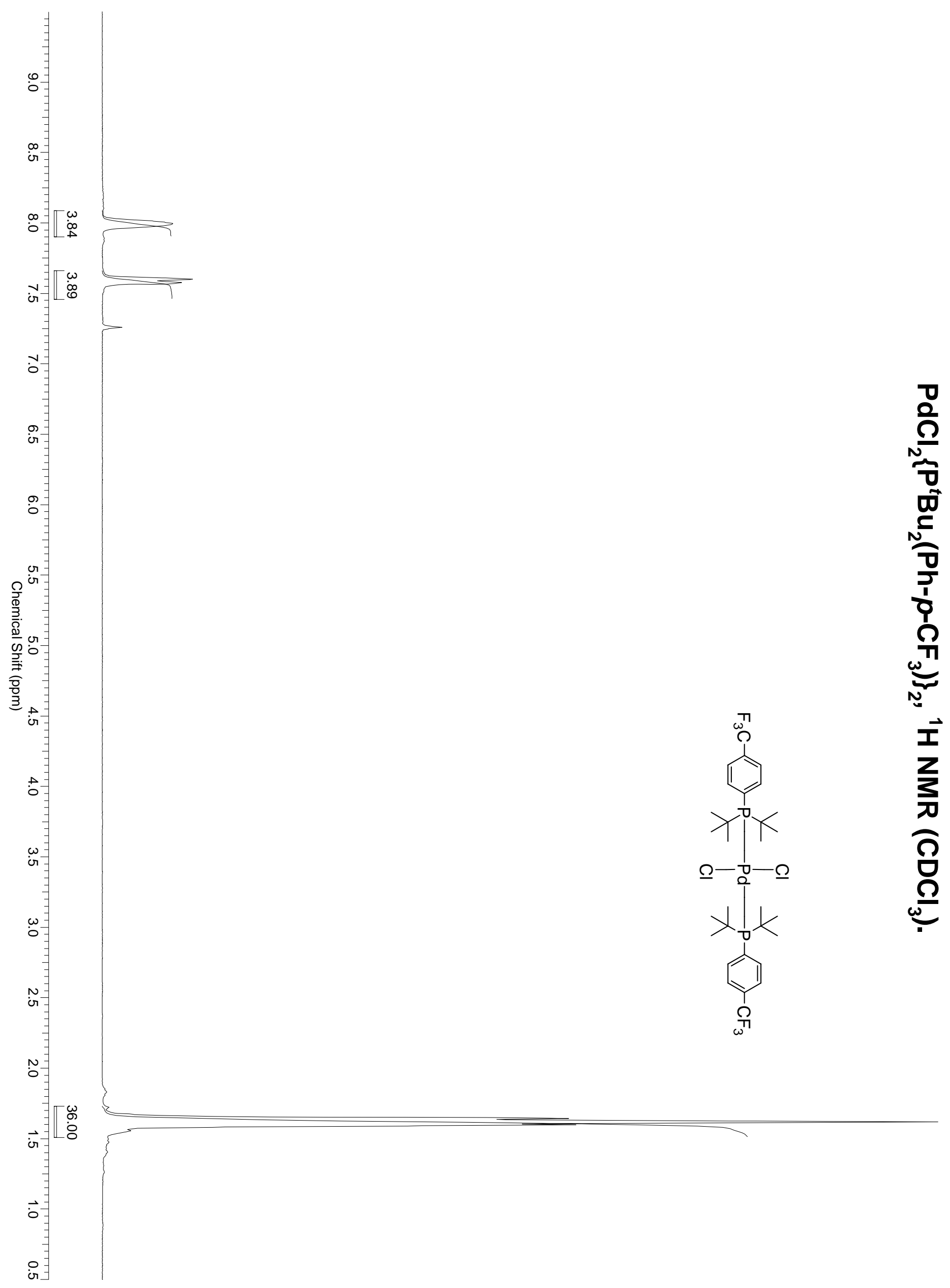




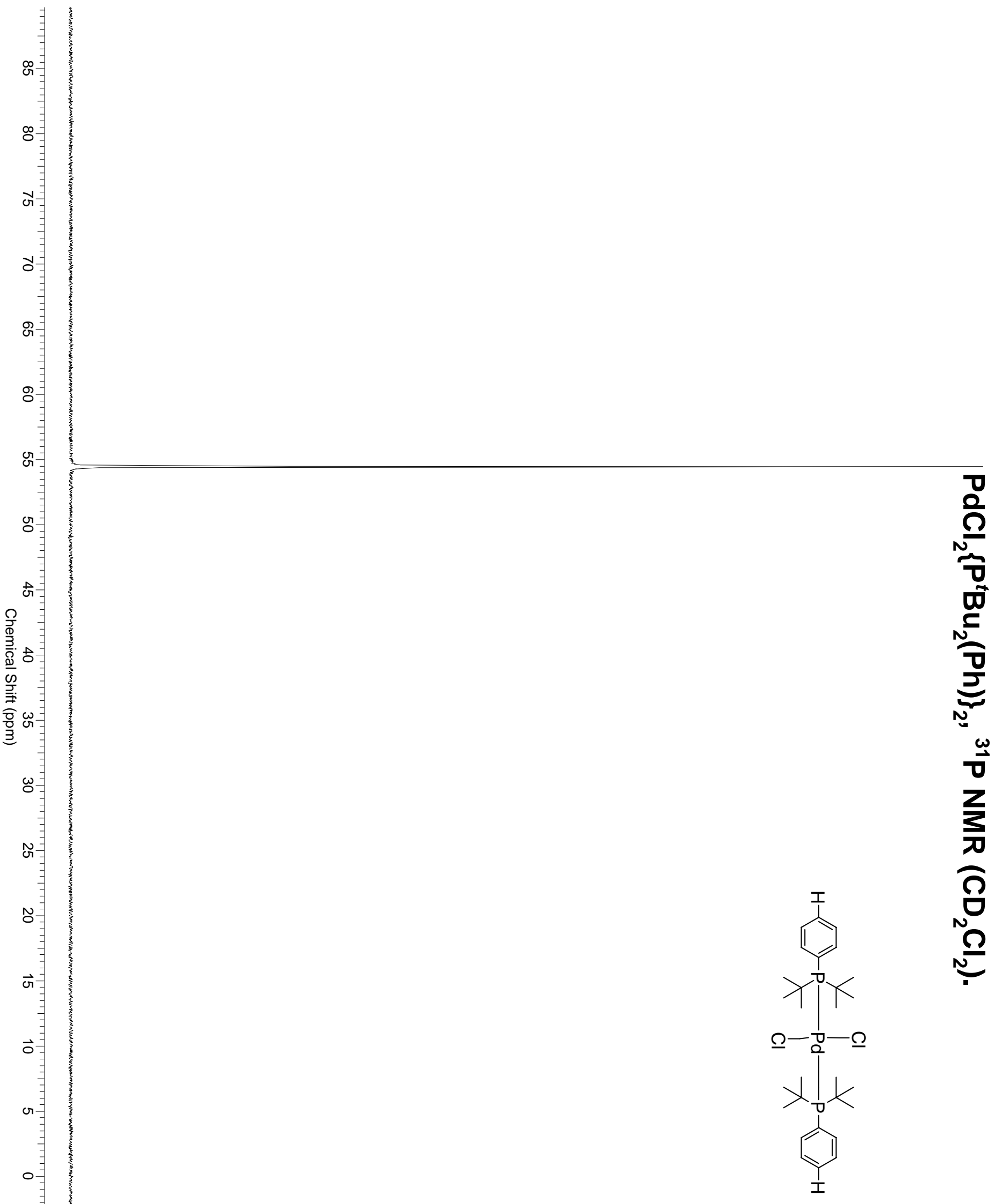




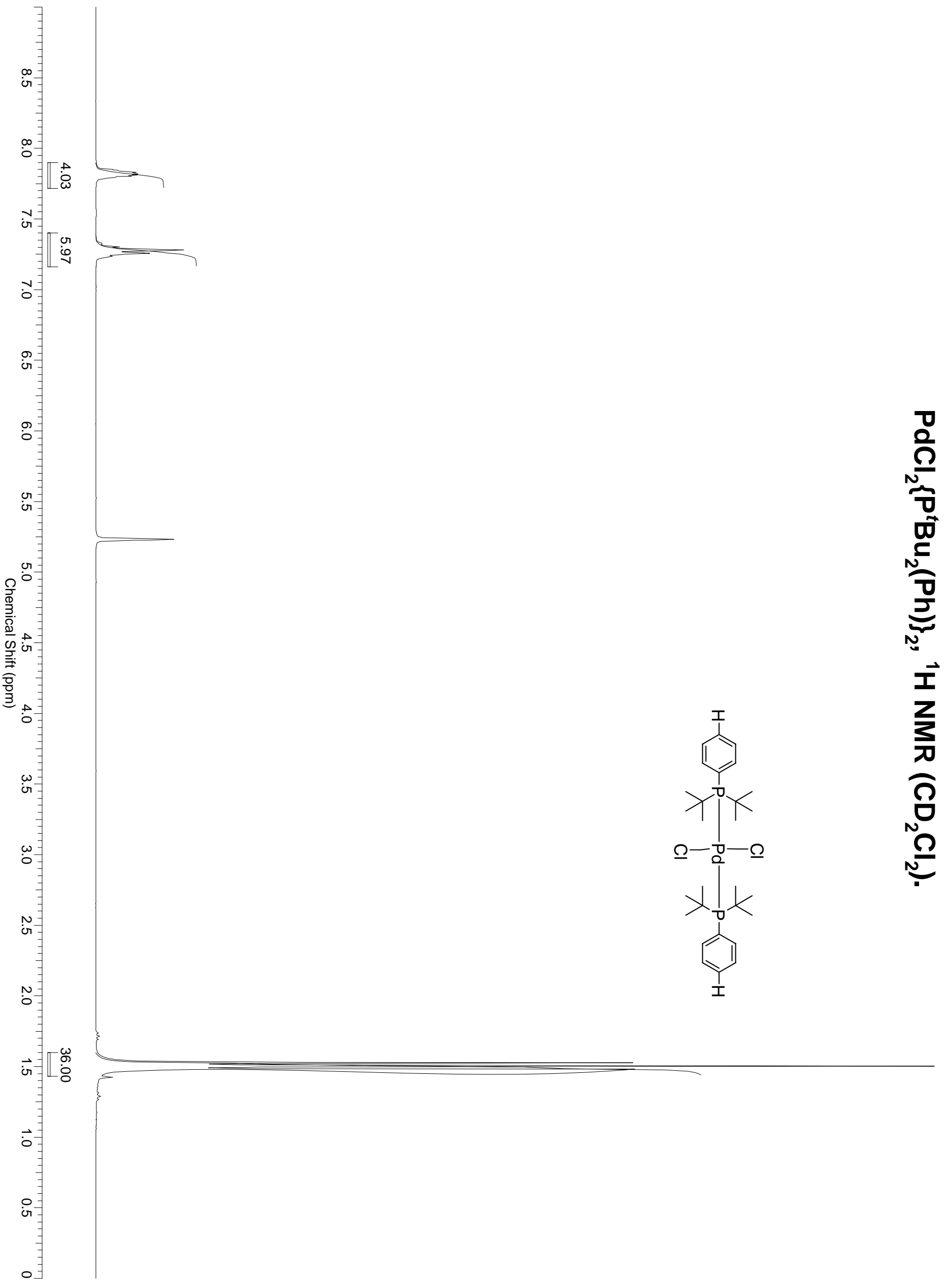




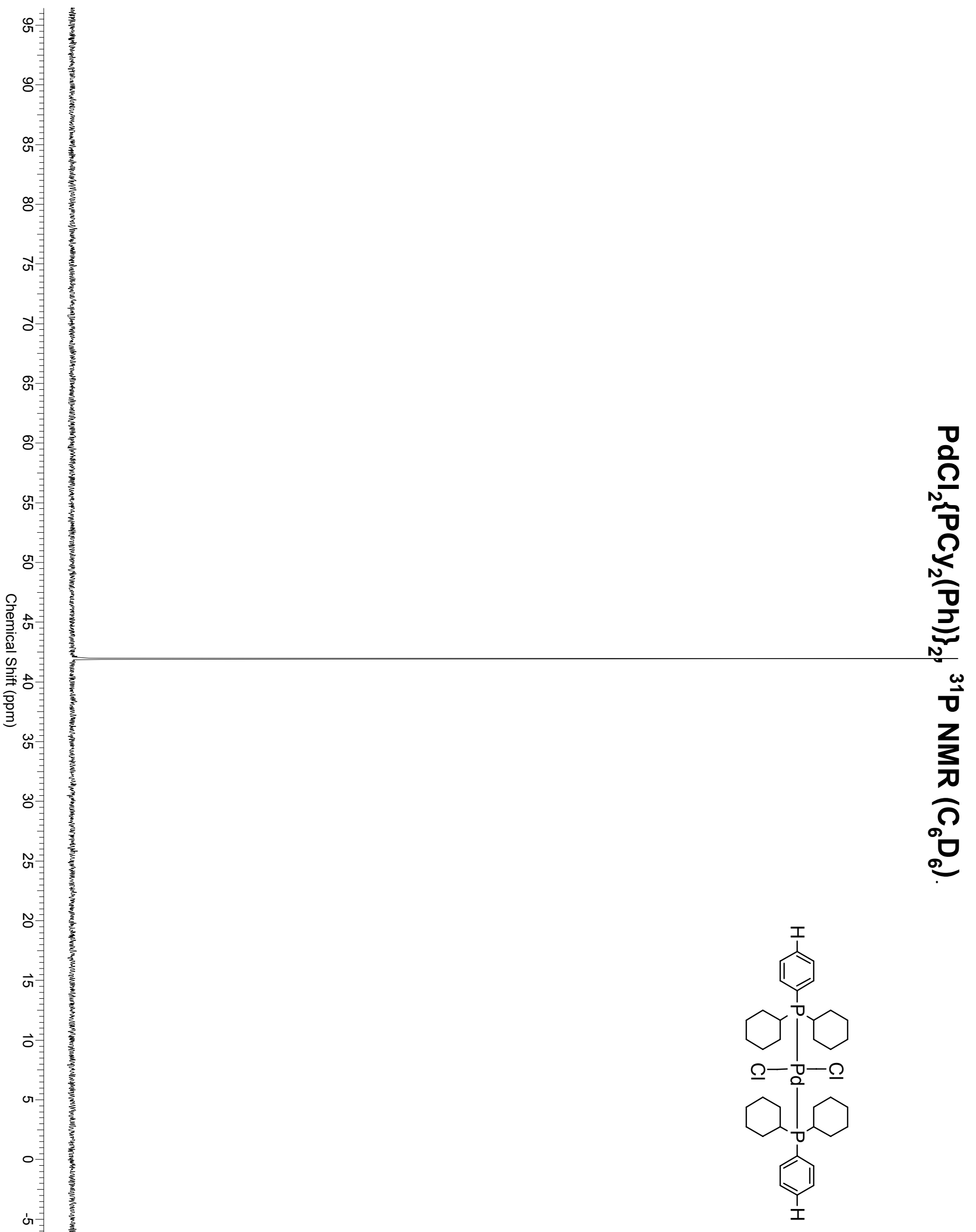




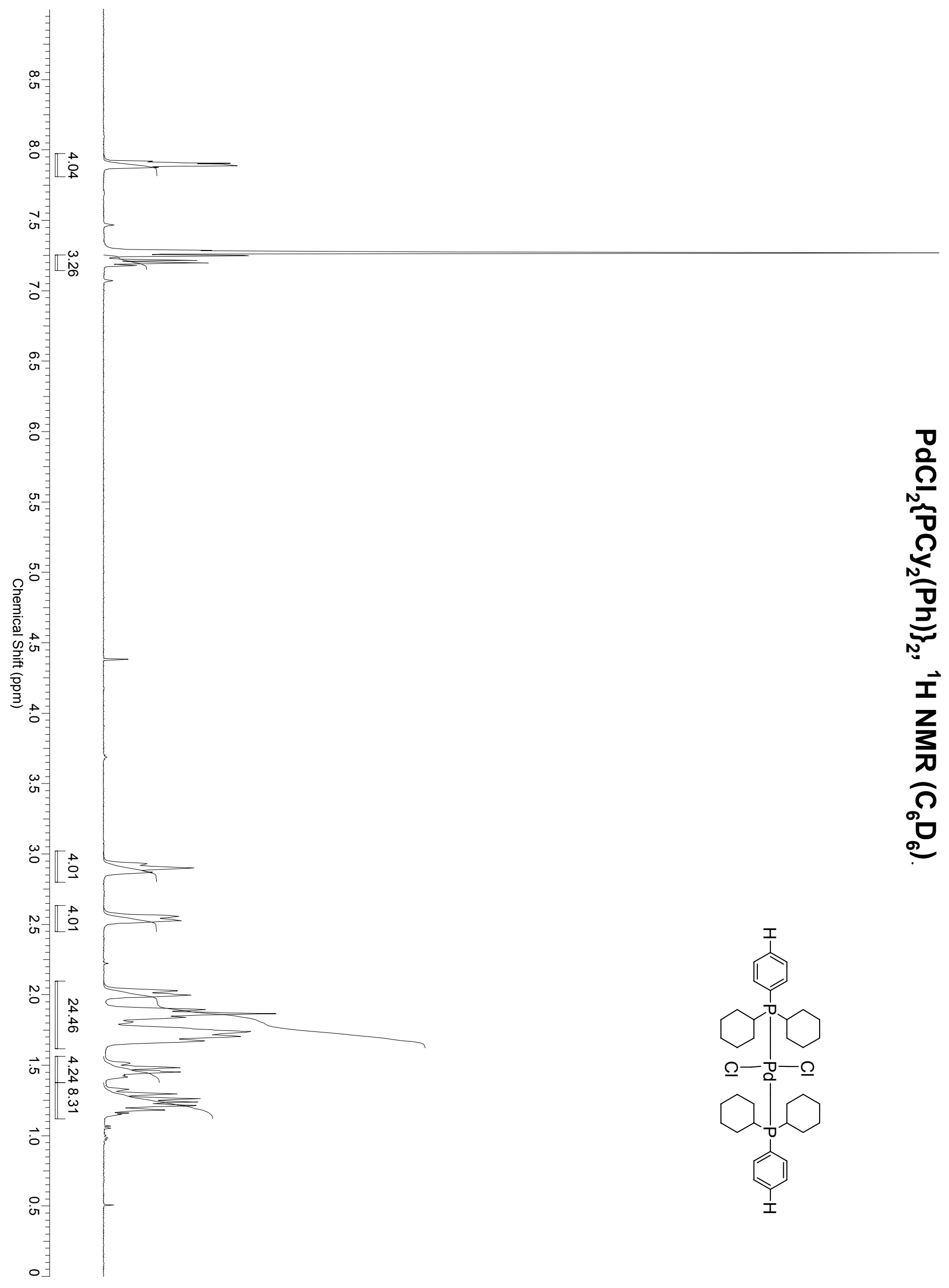




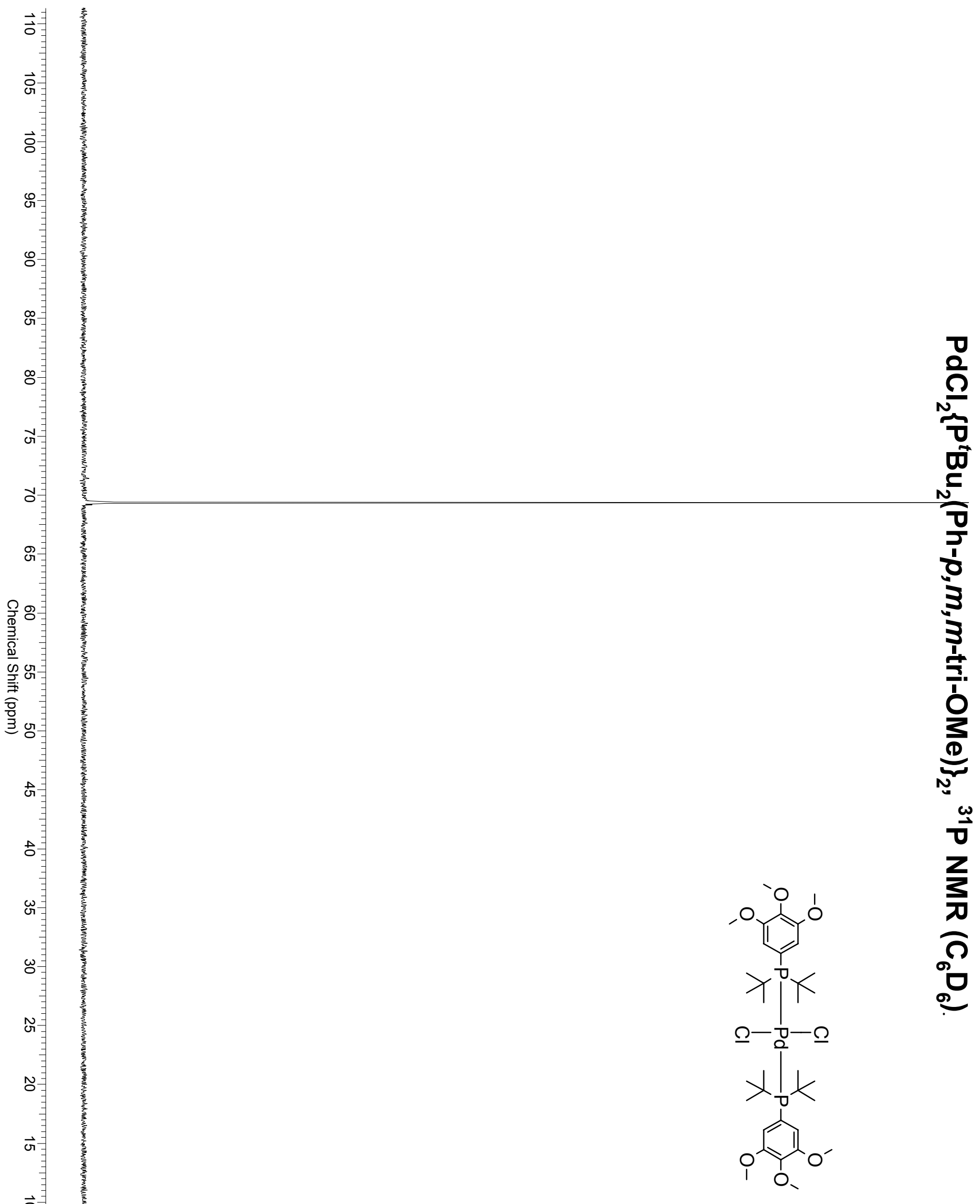




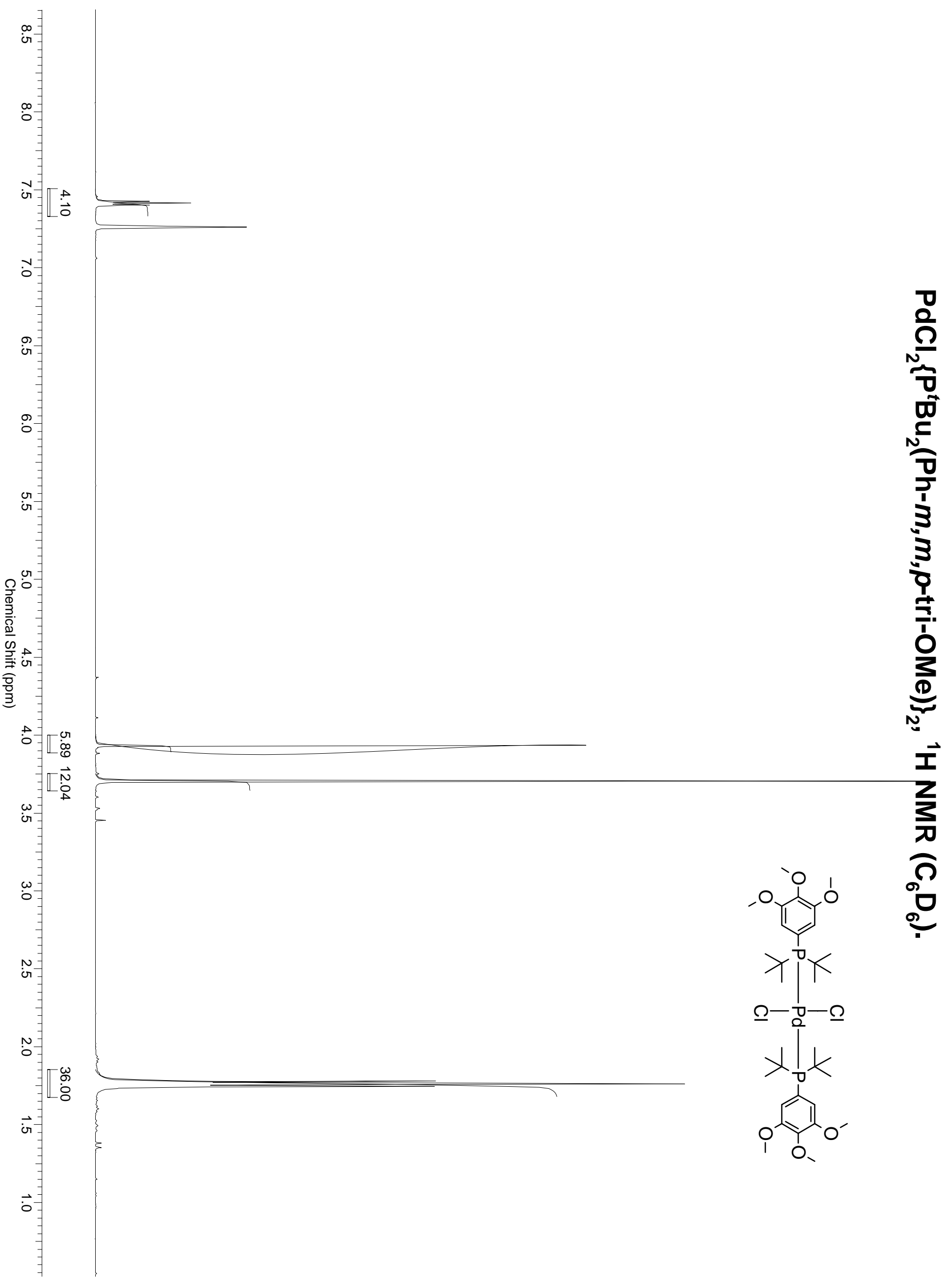




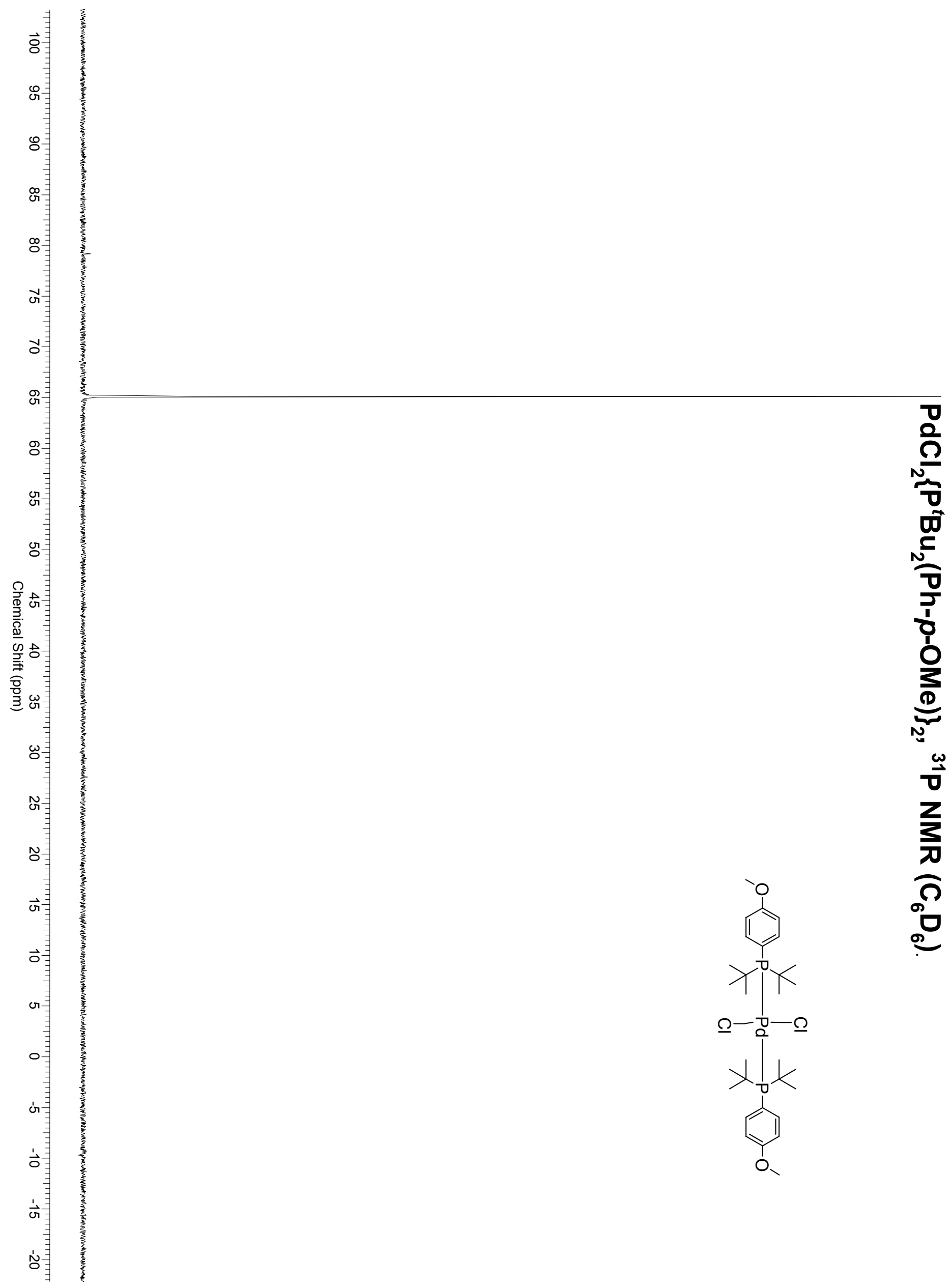




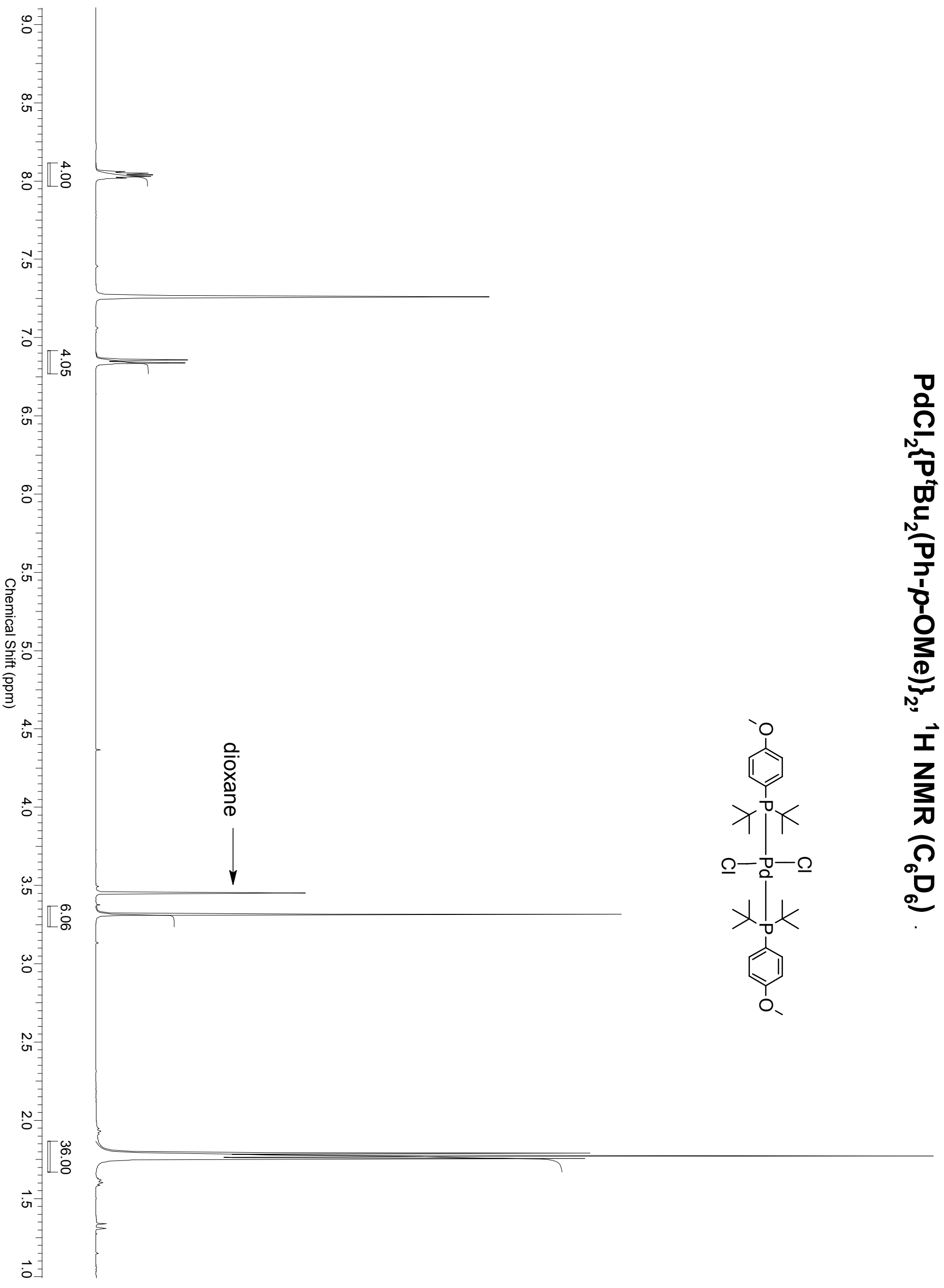




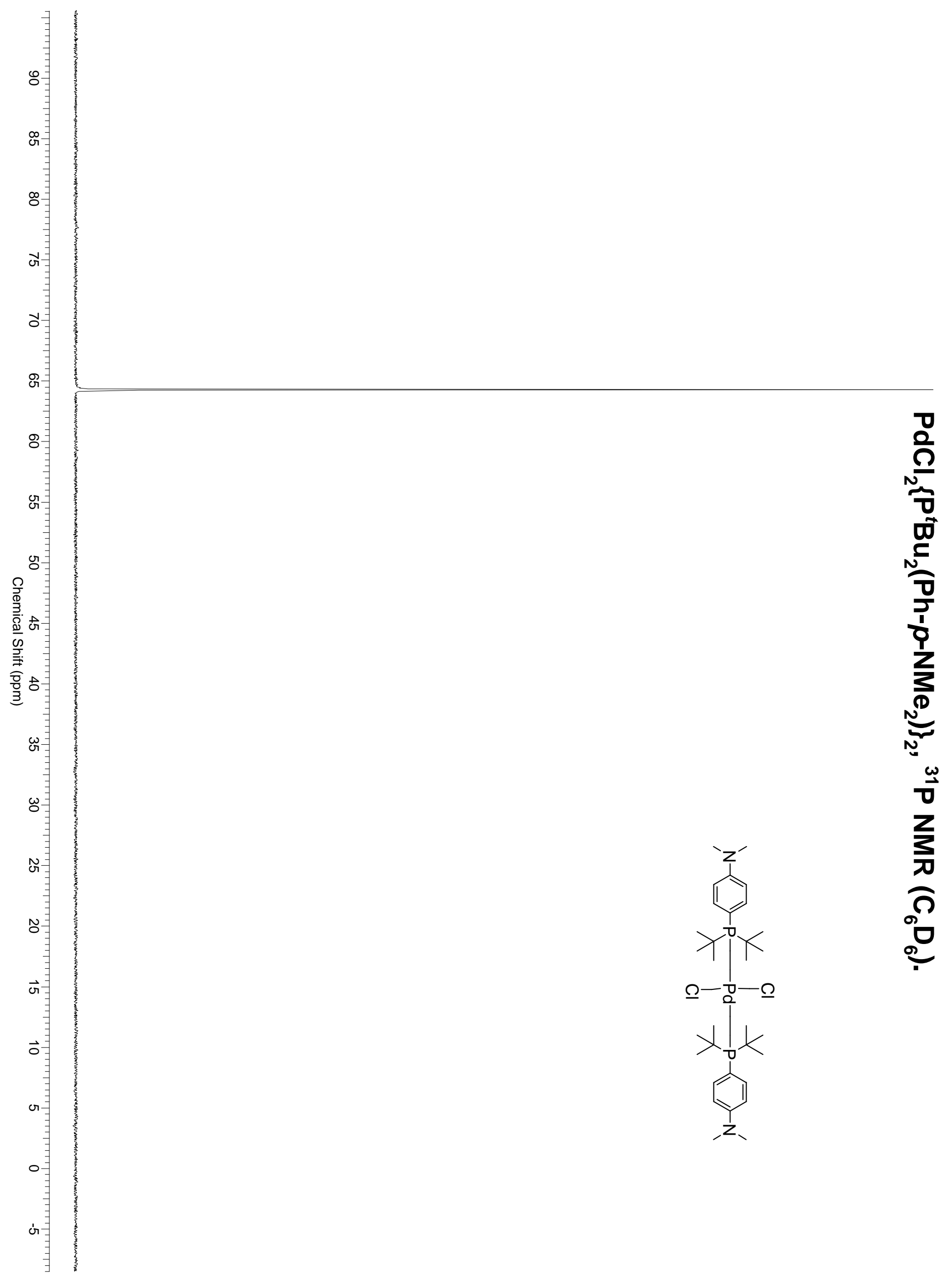




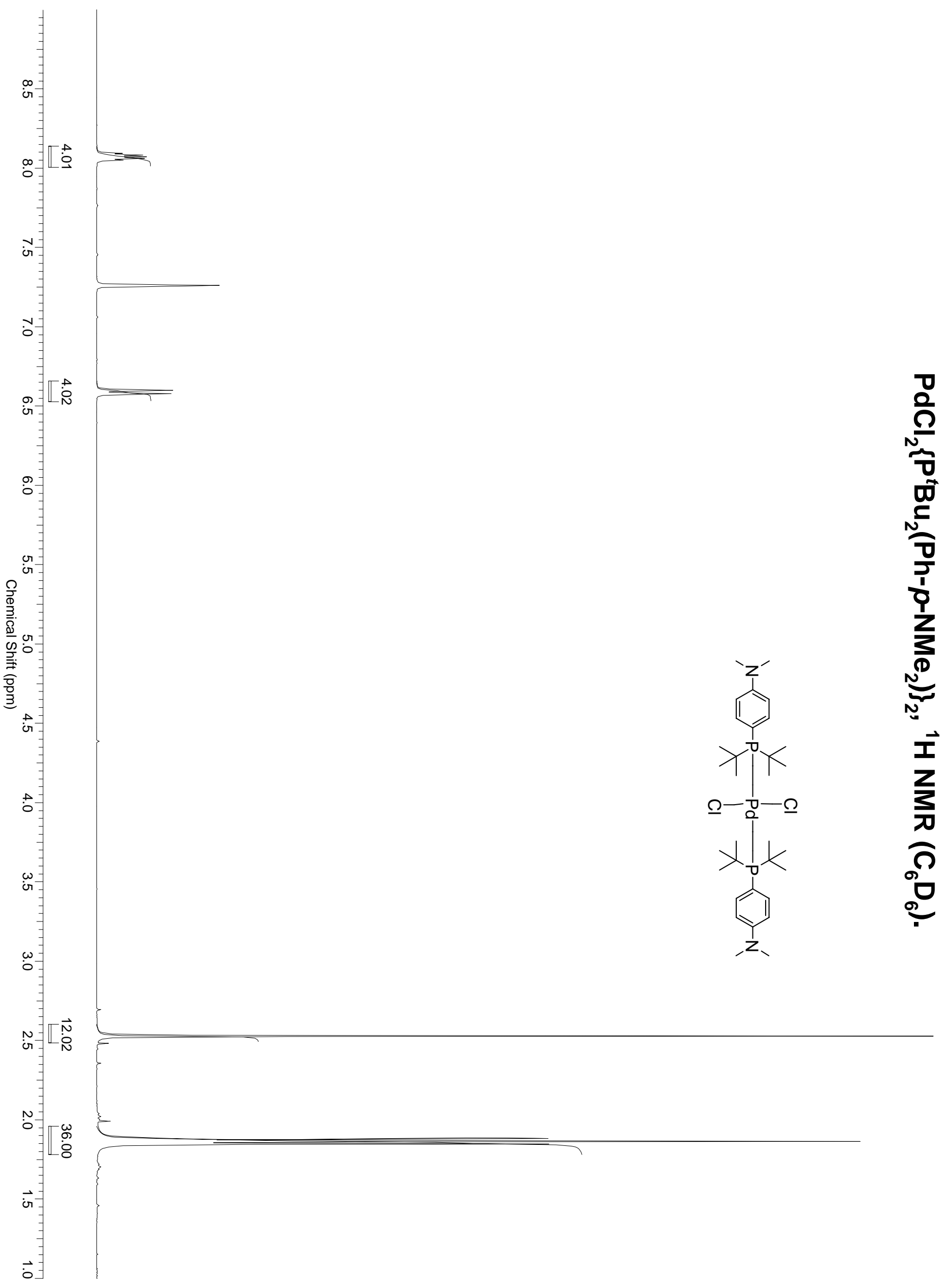




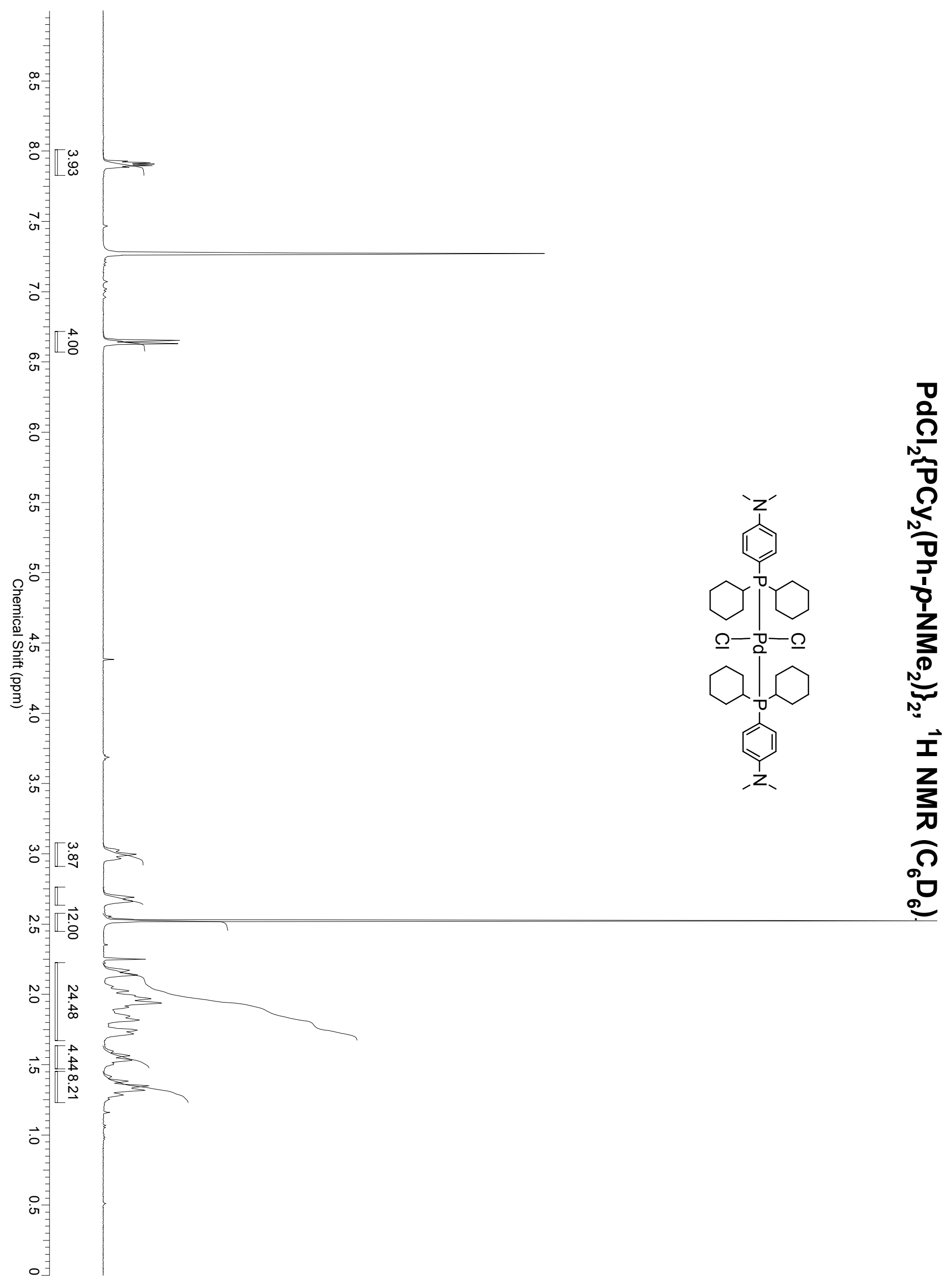




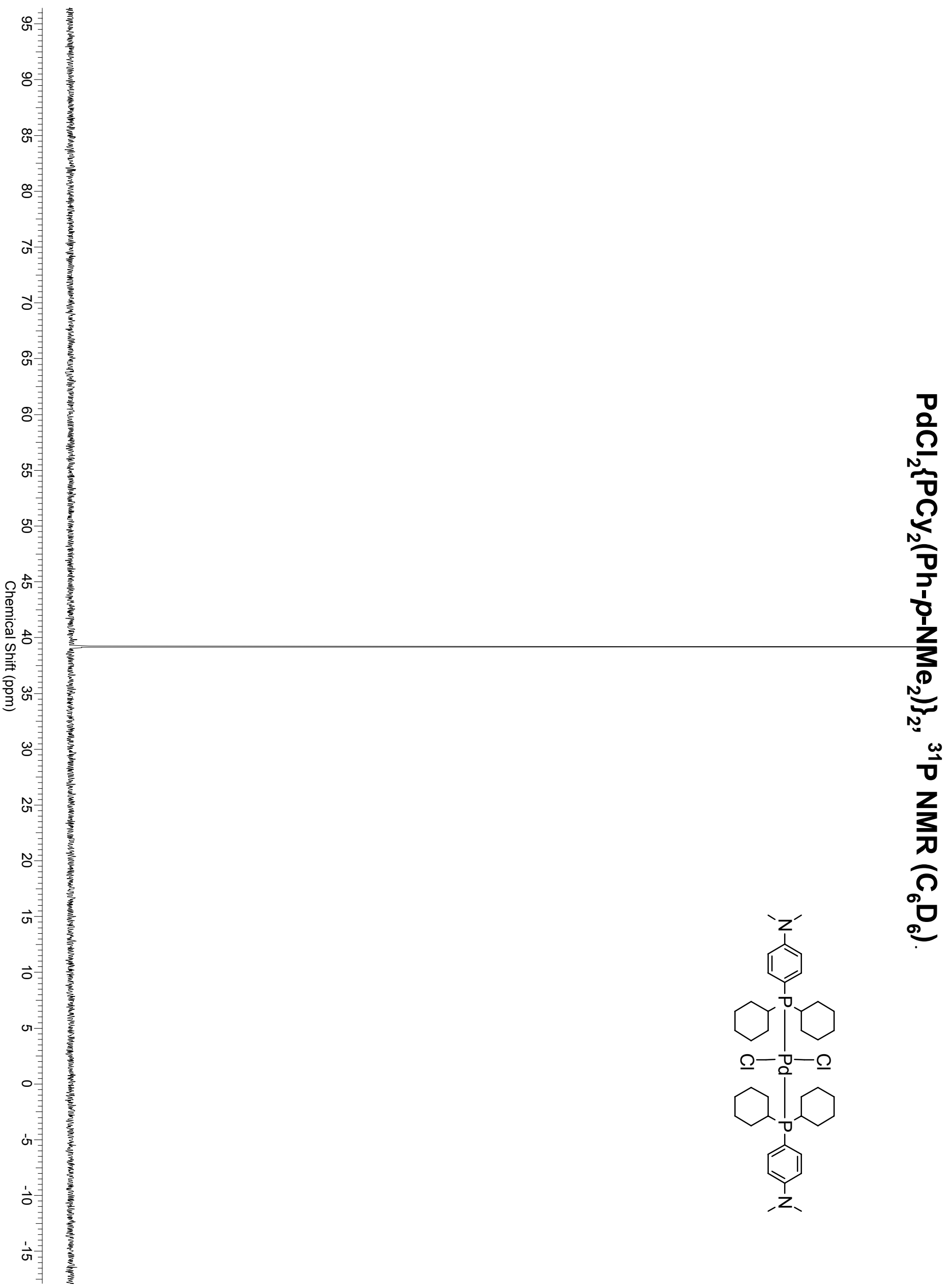




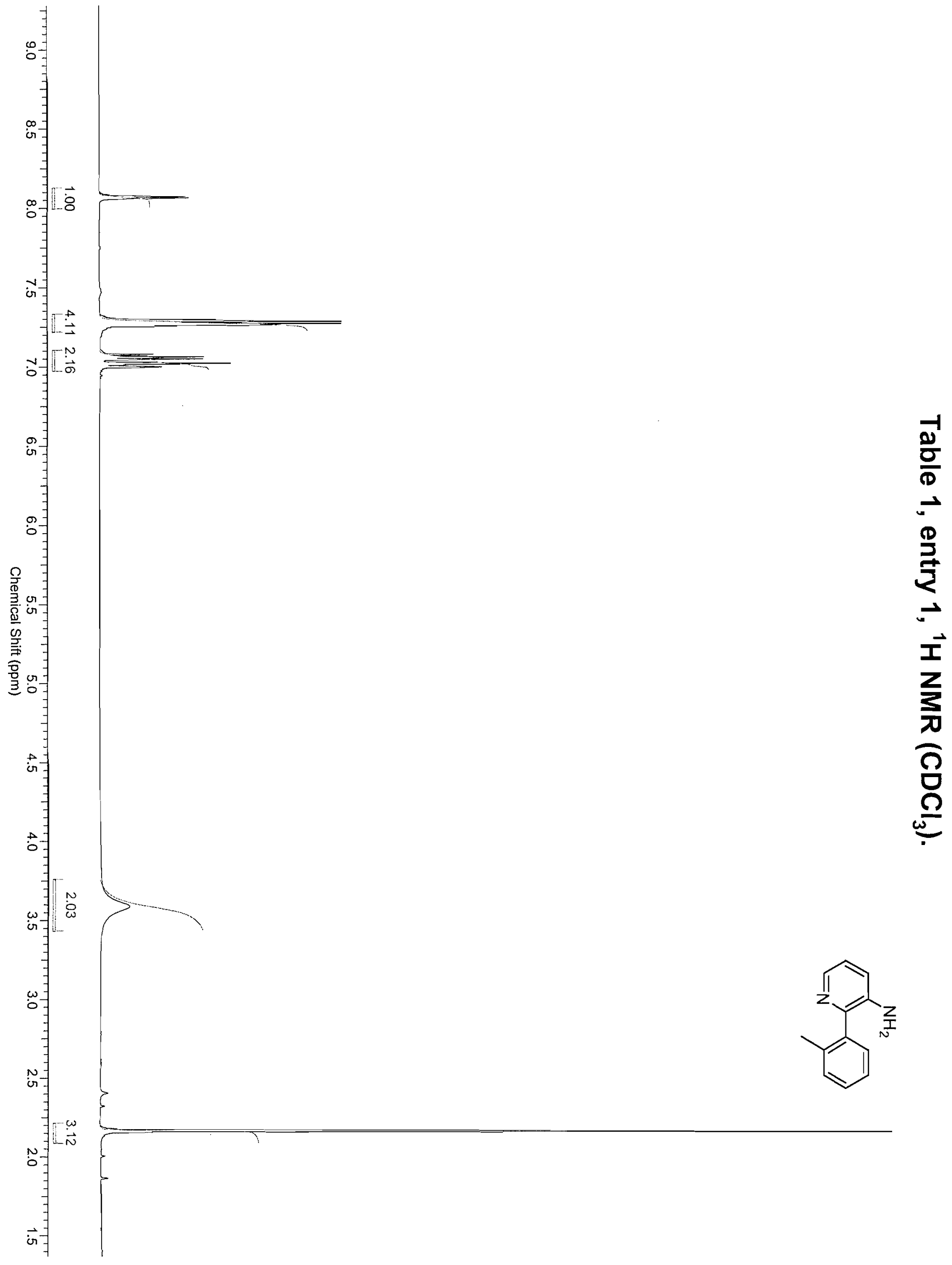




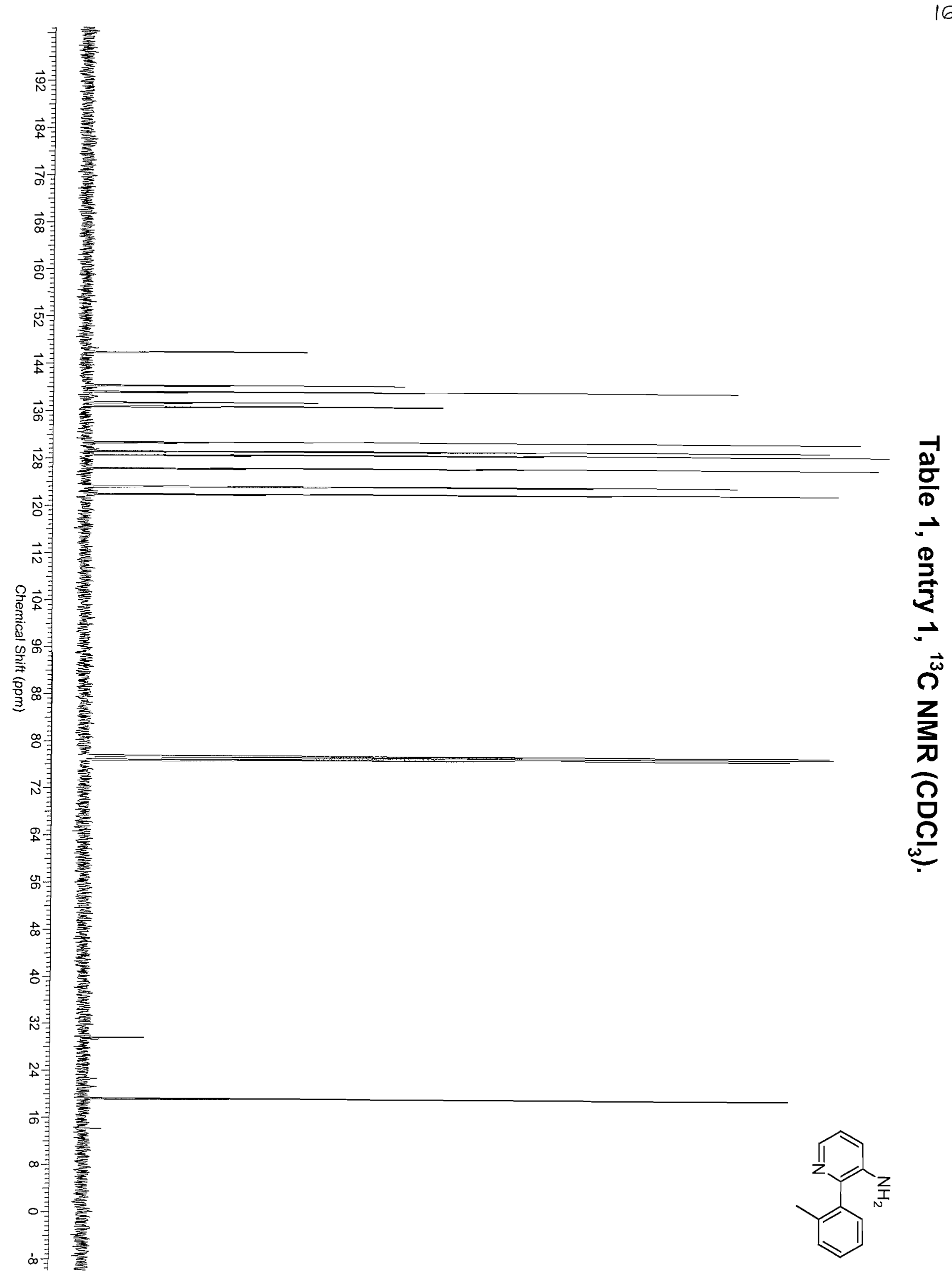




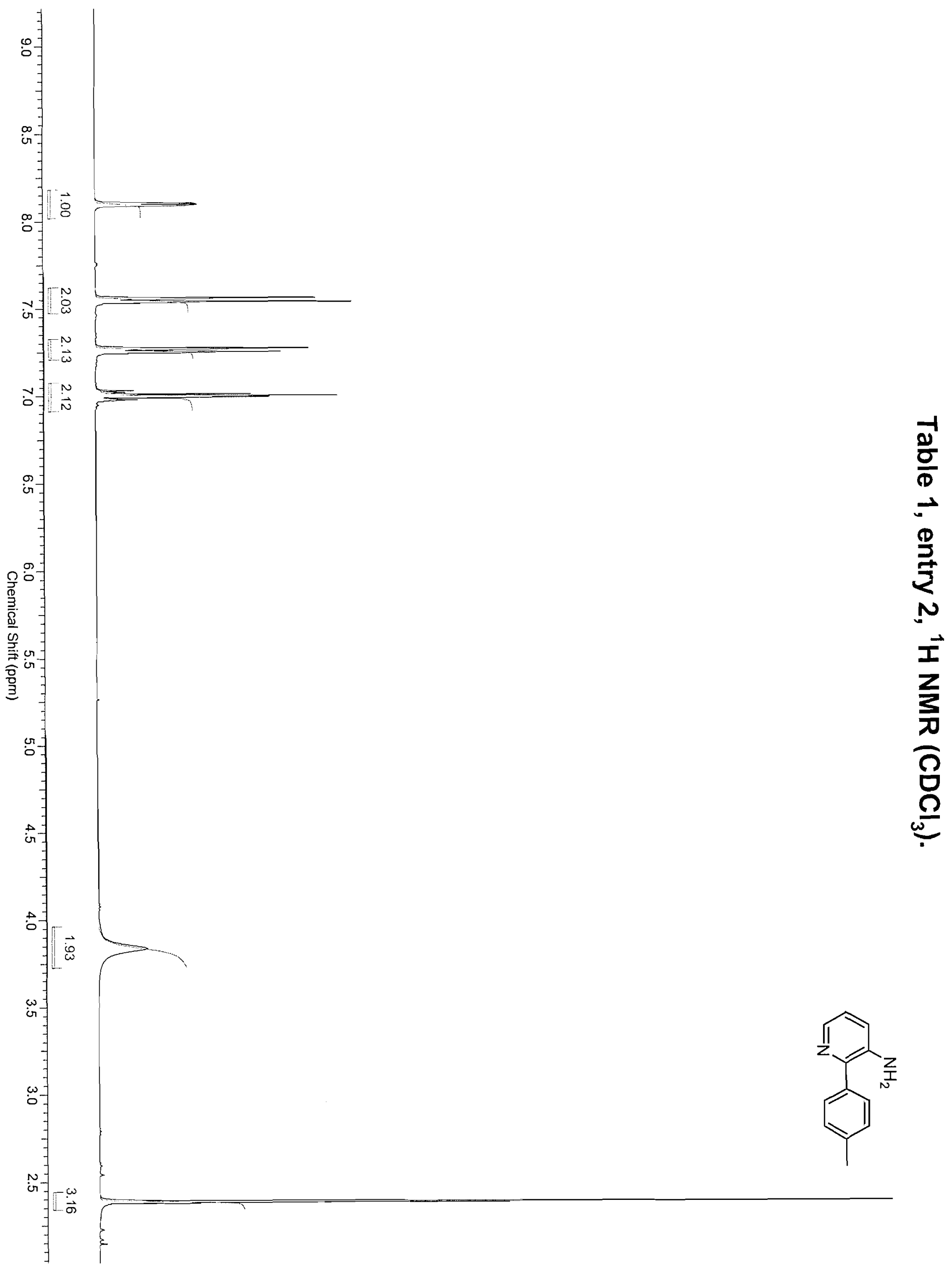




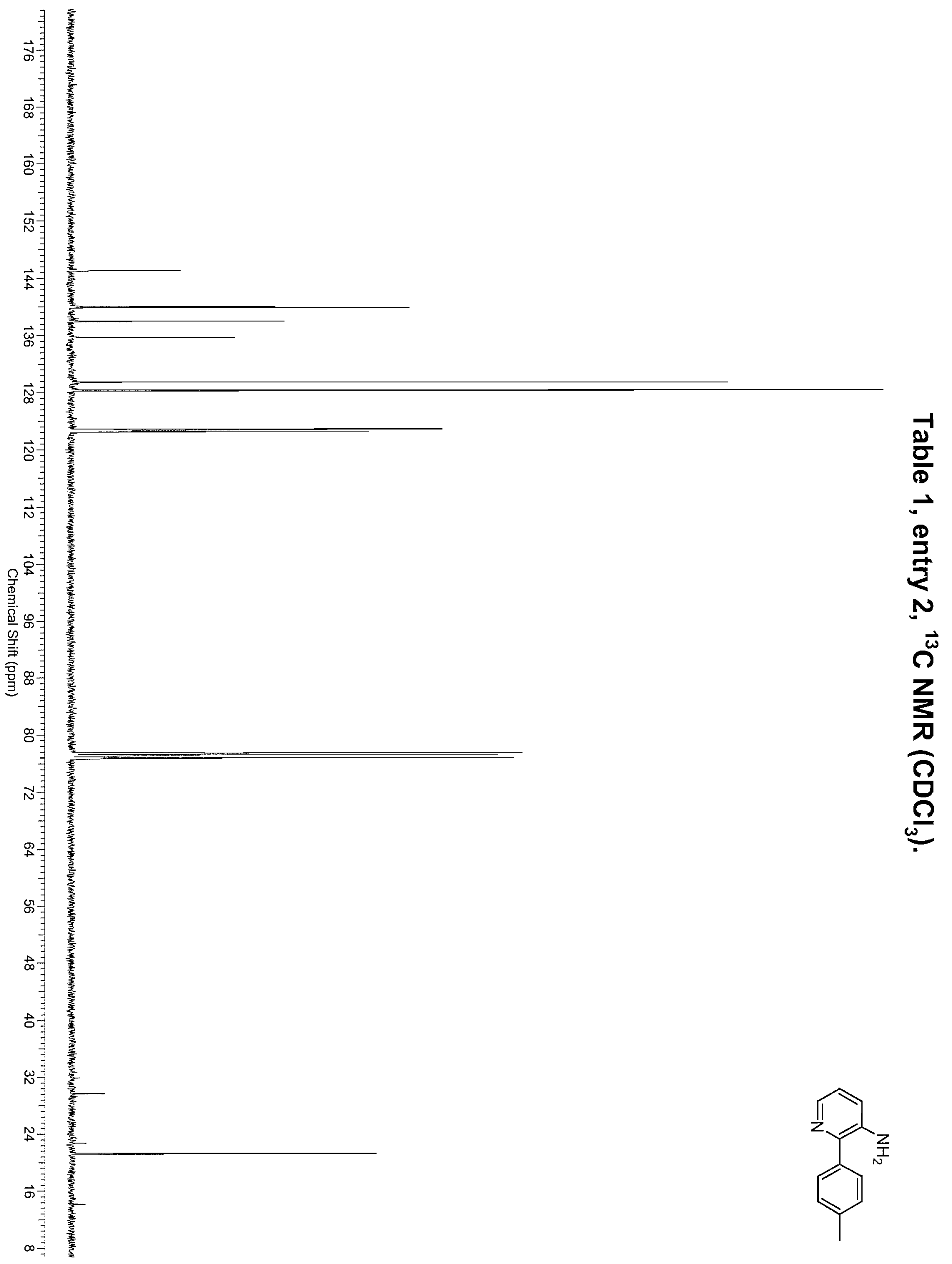




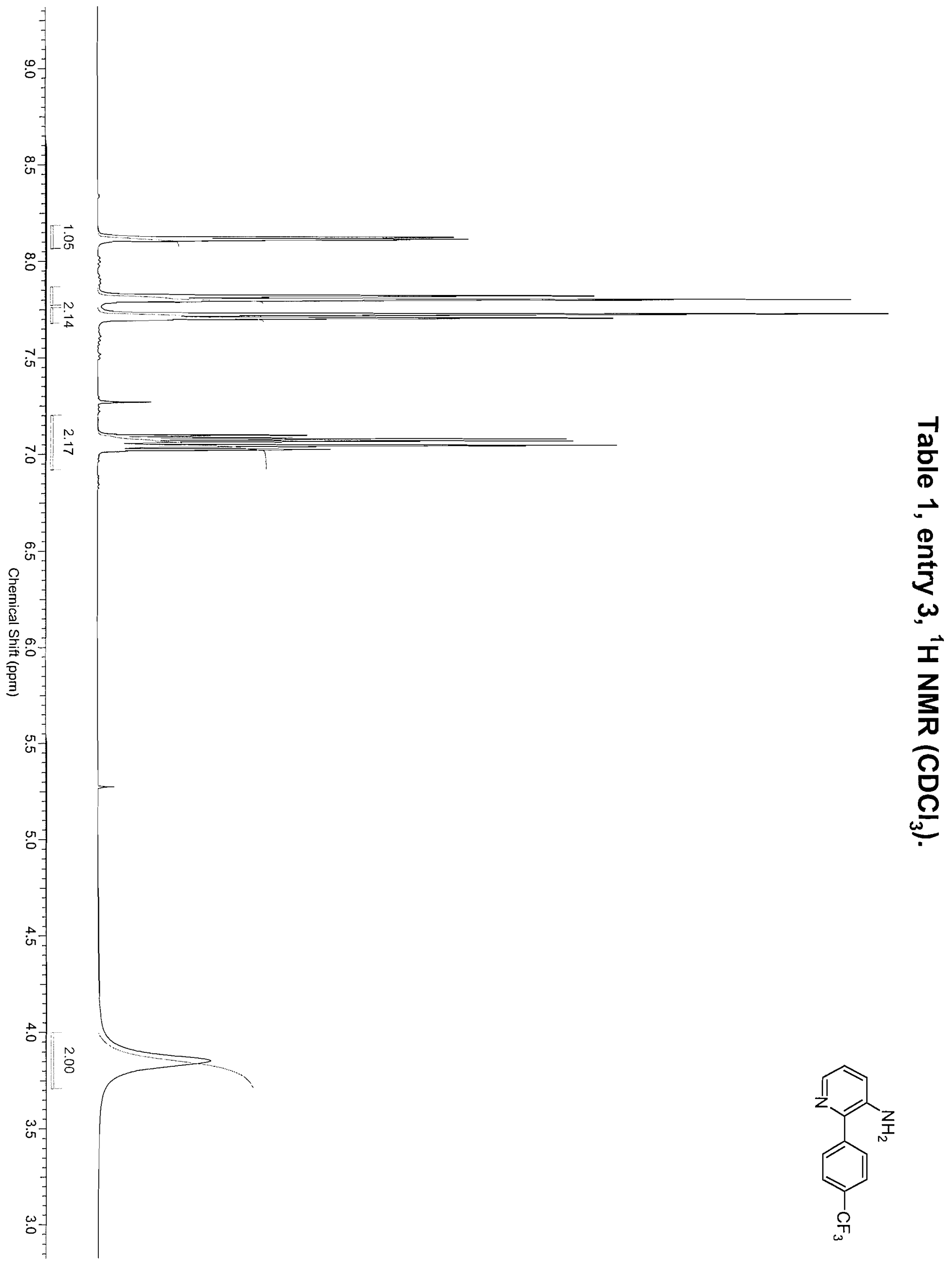




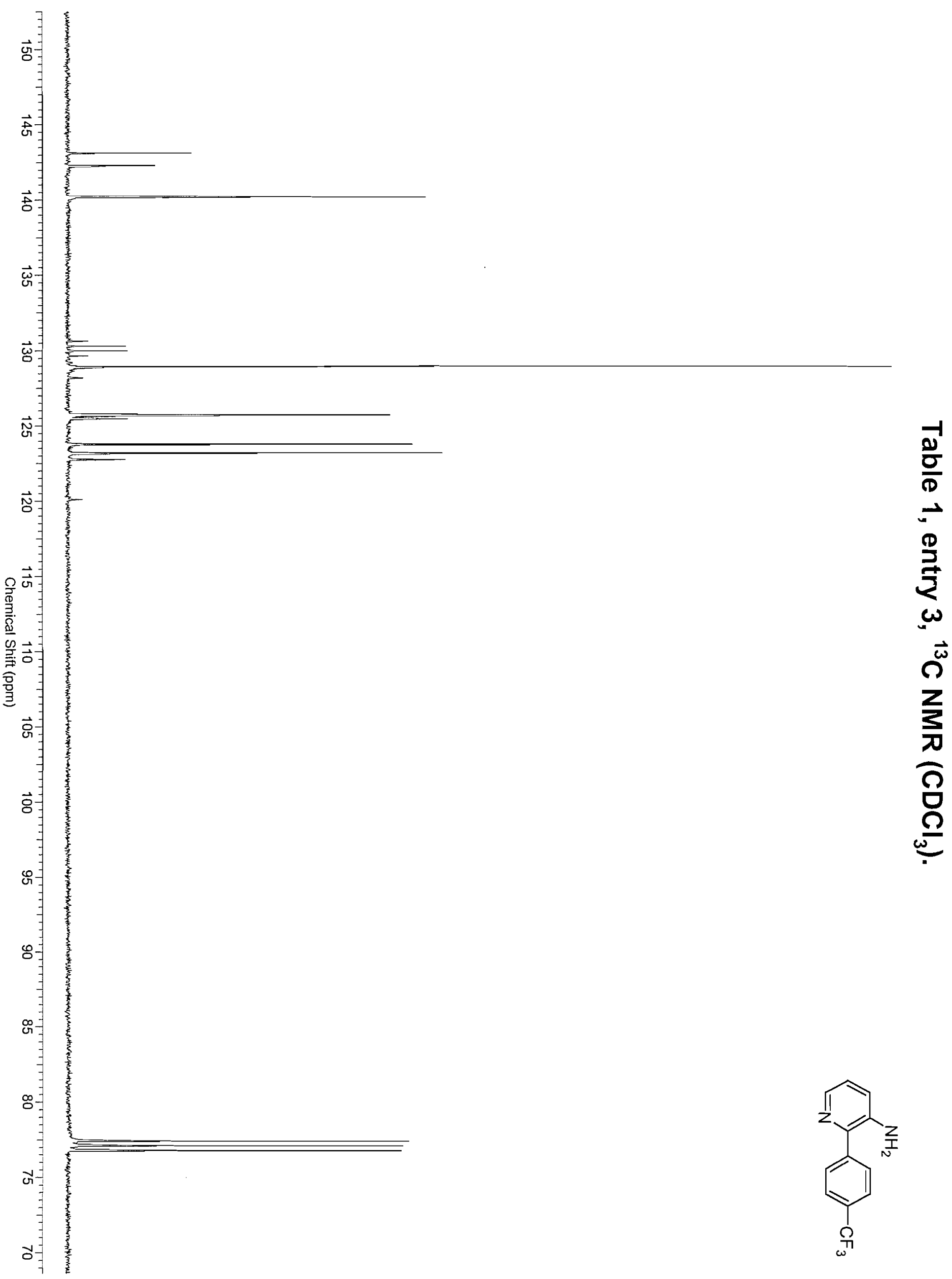



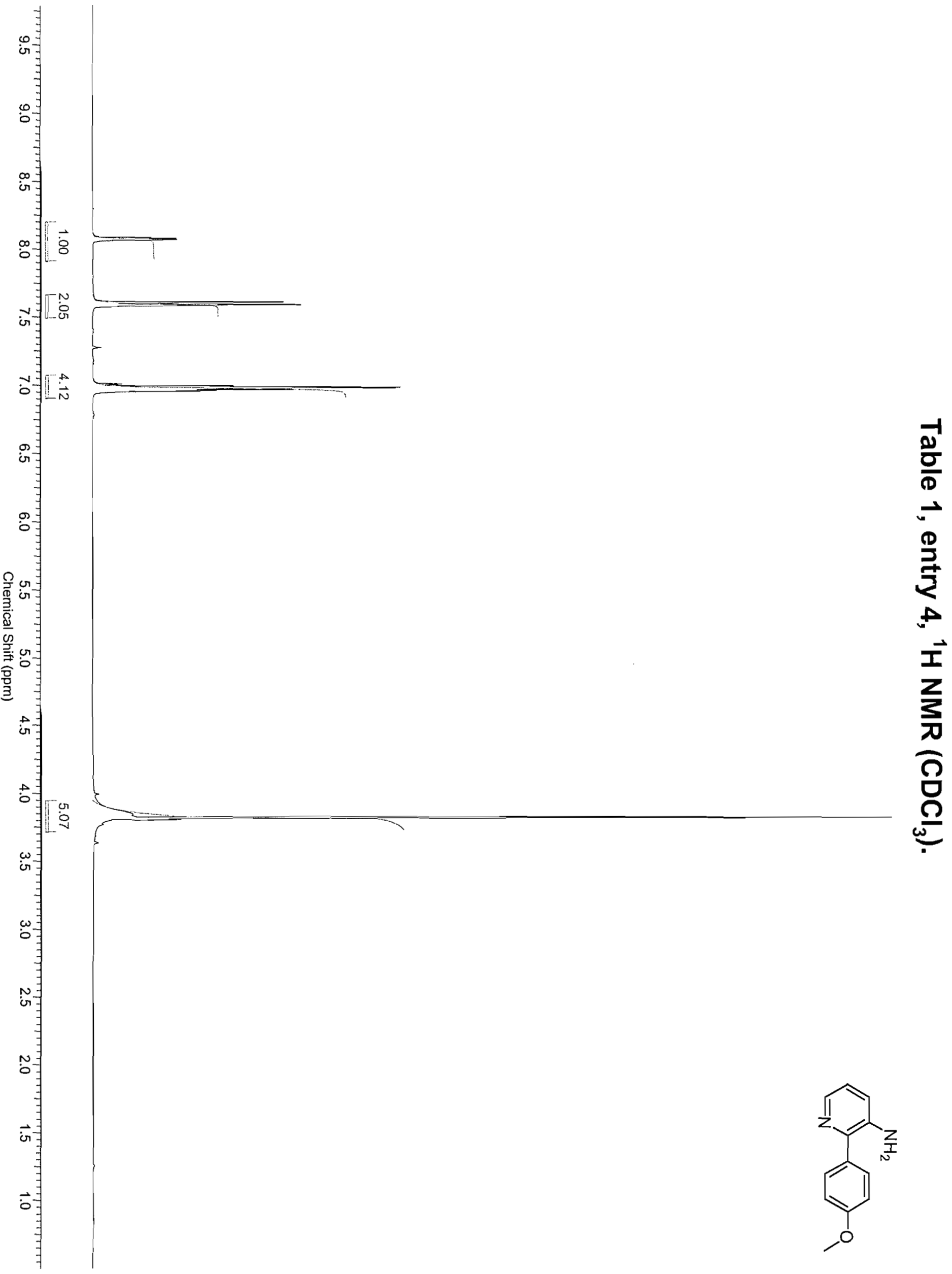


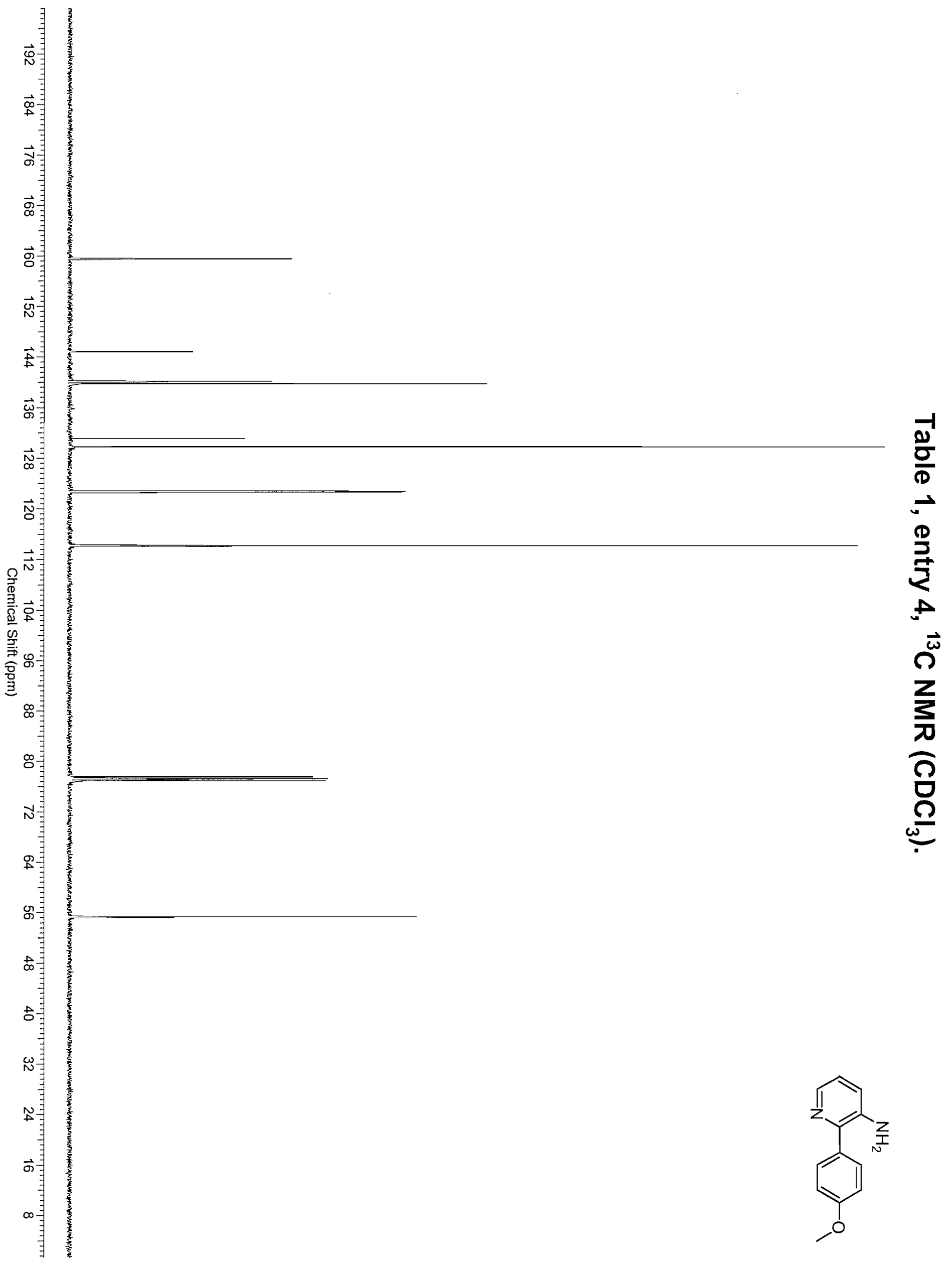




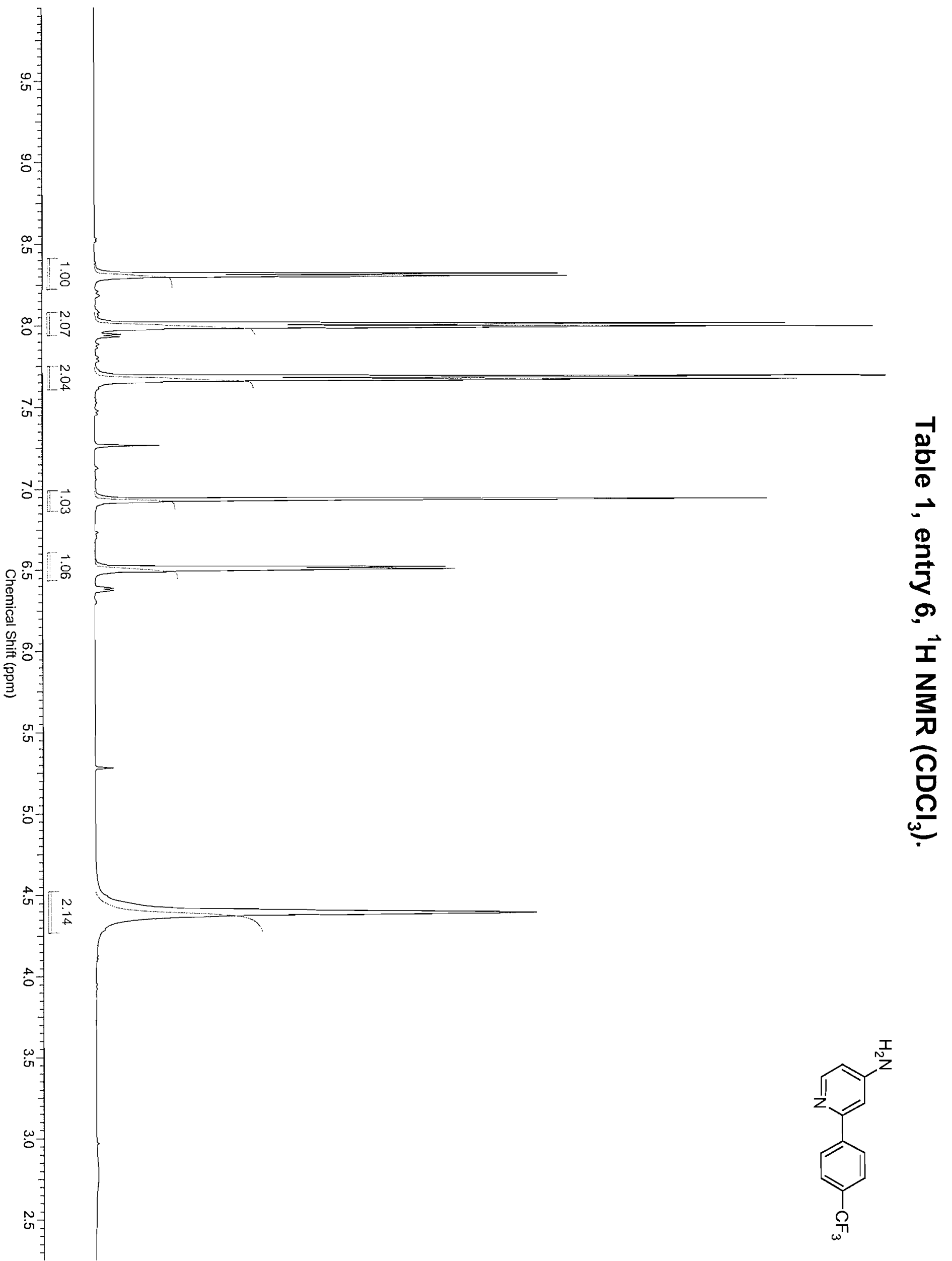


34
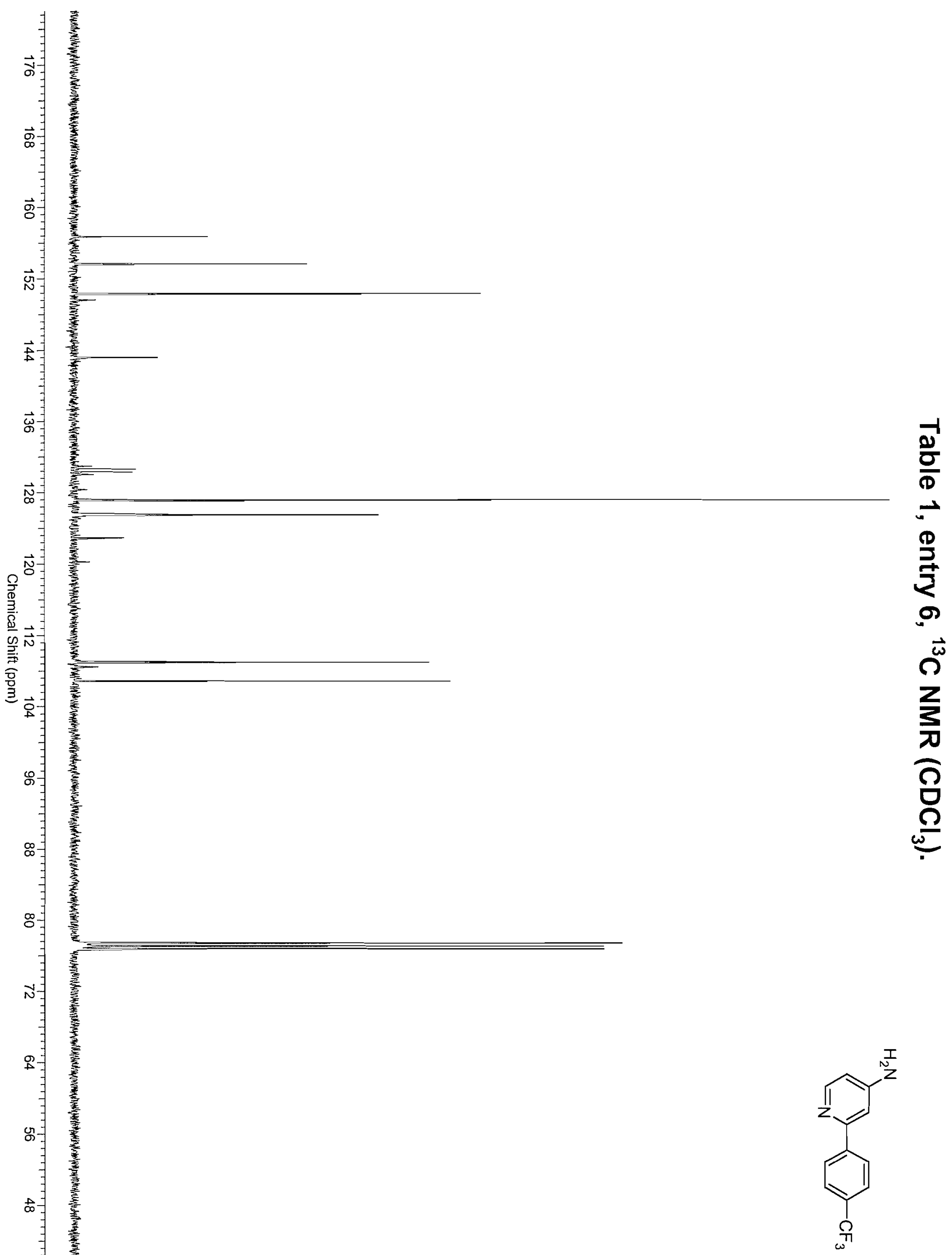


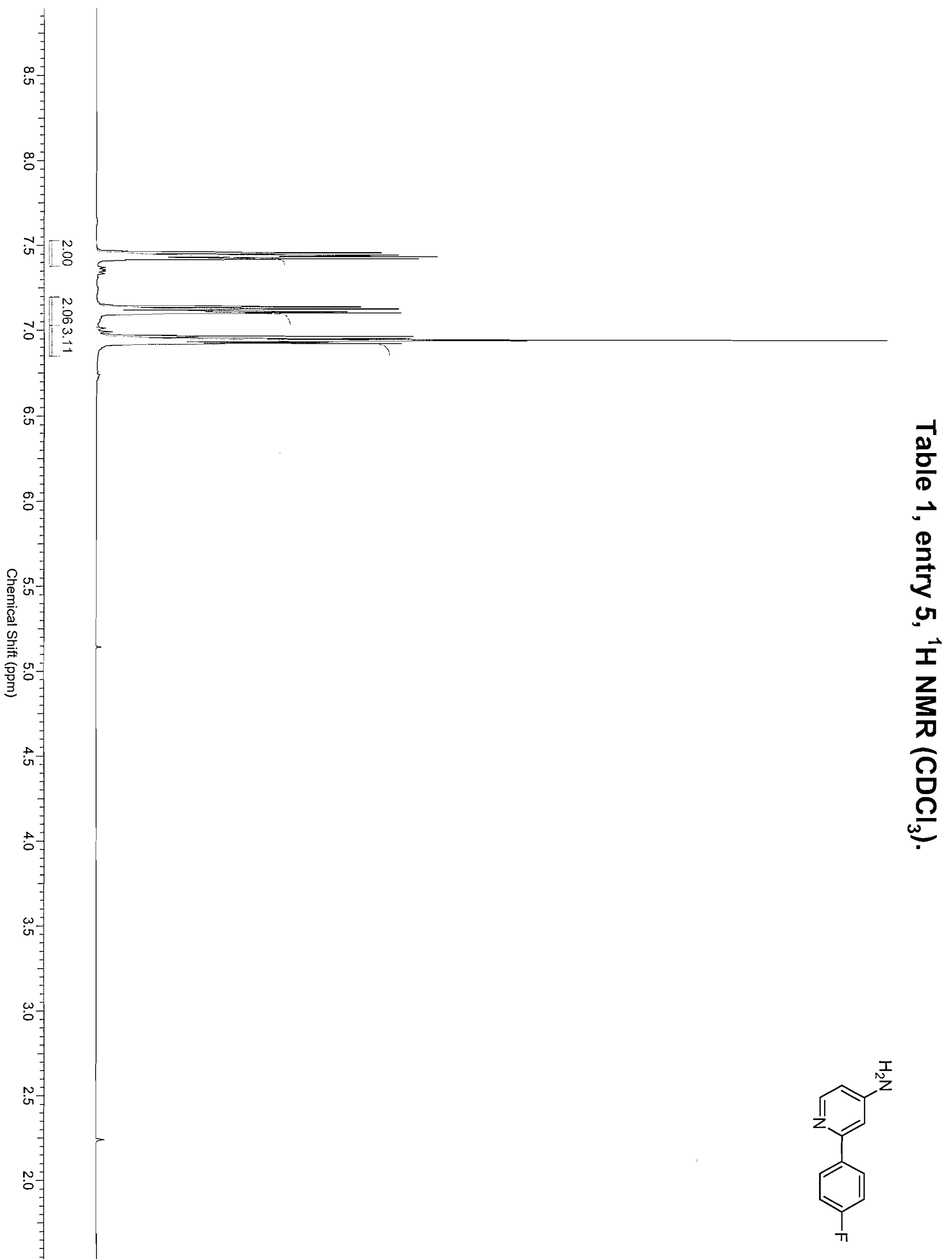




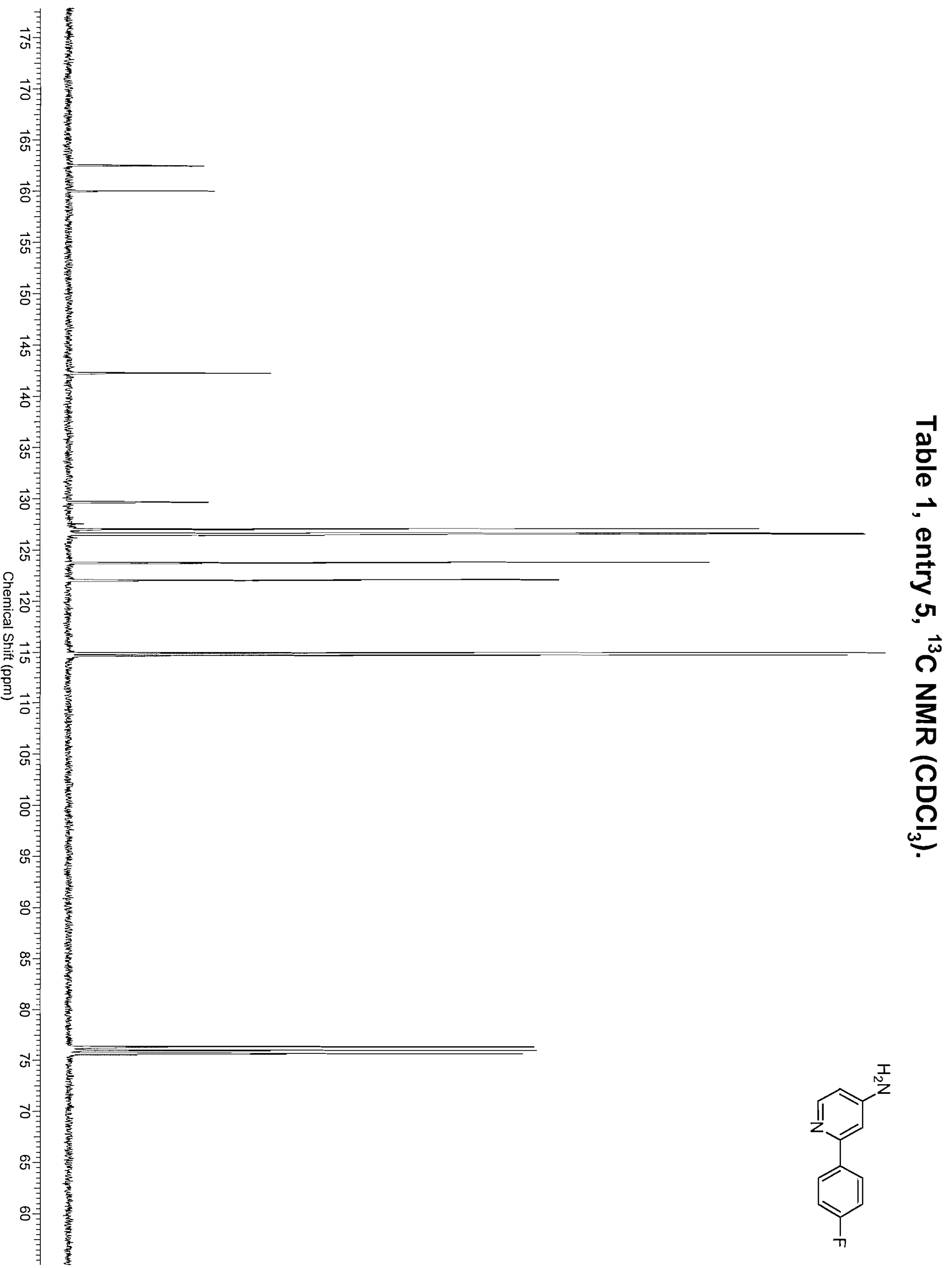




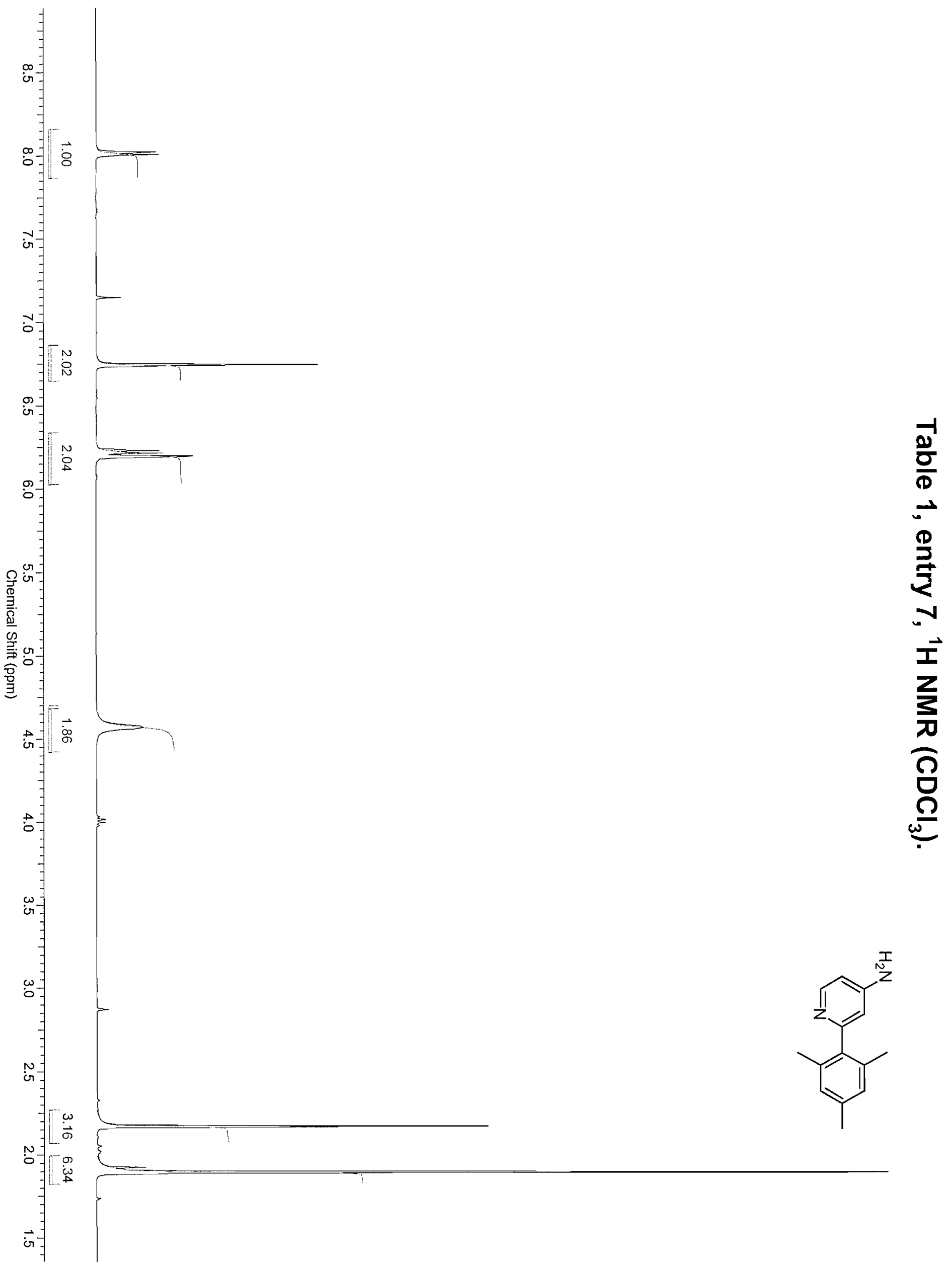




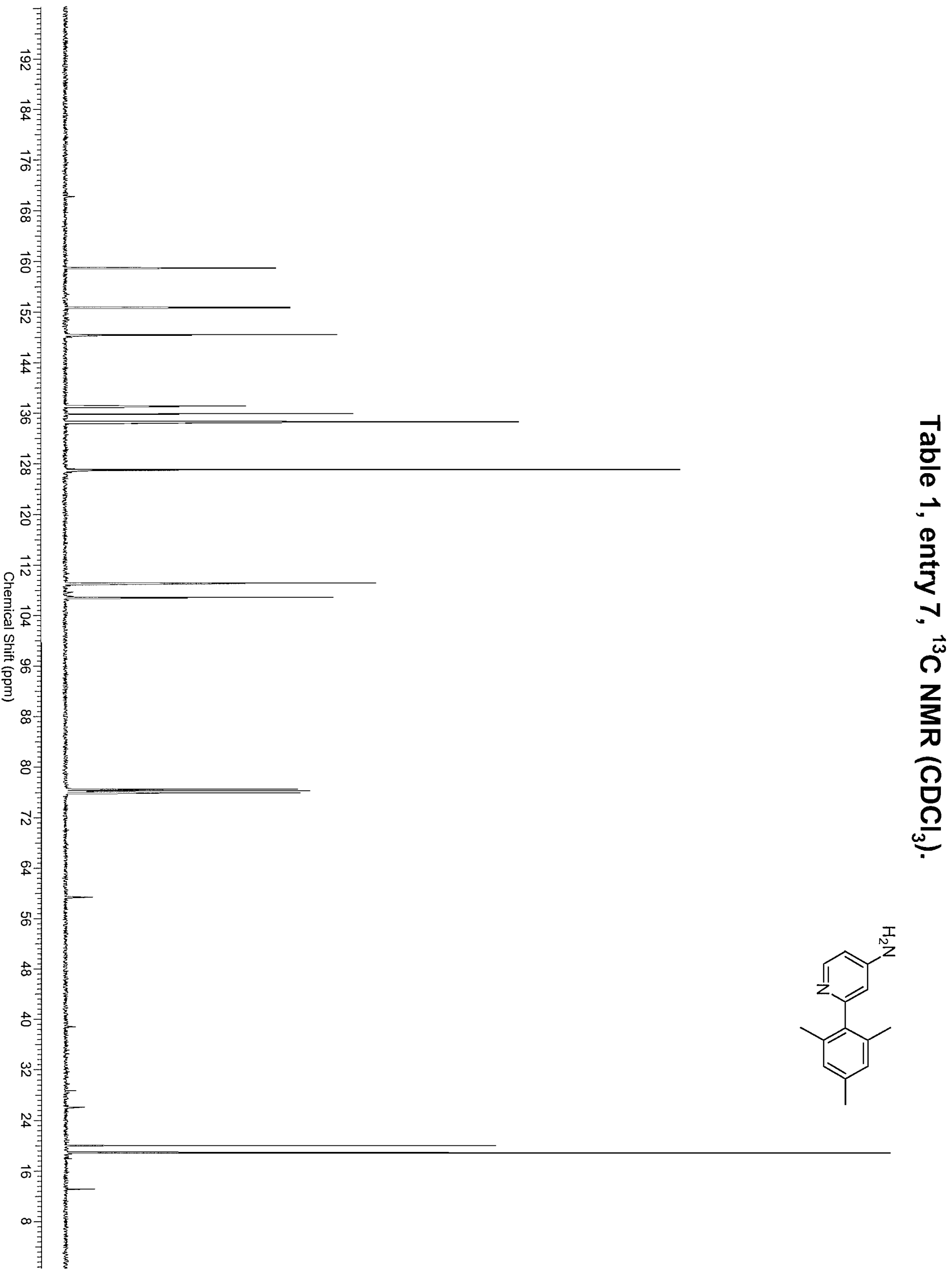




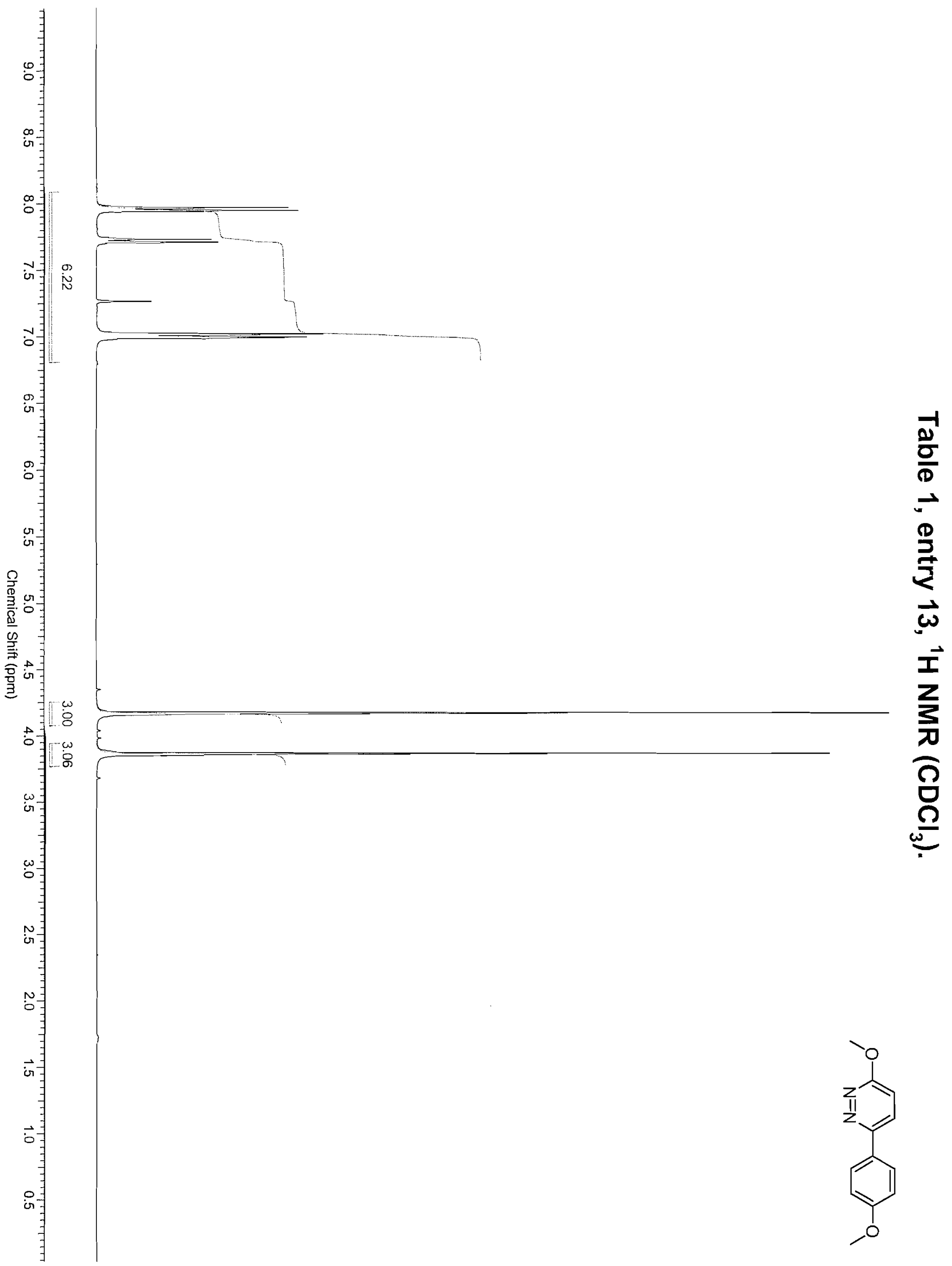




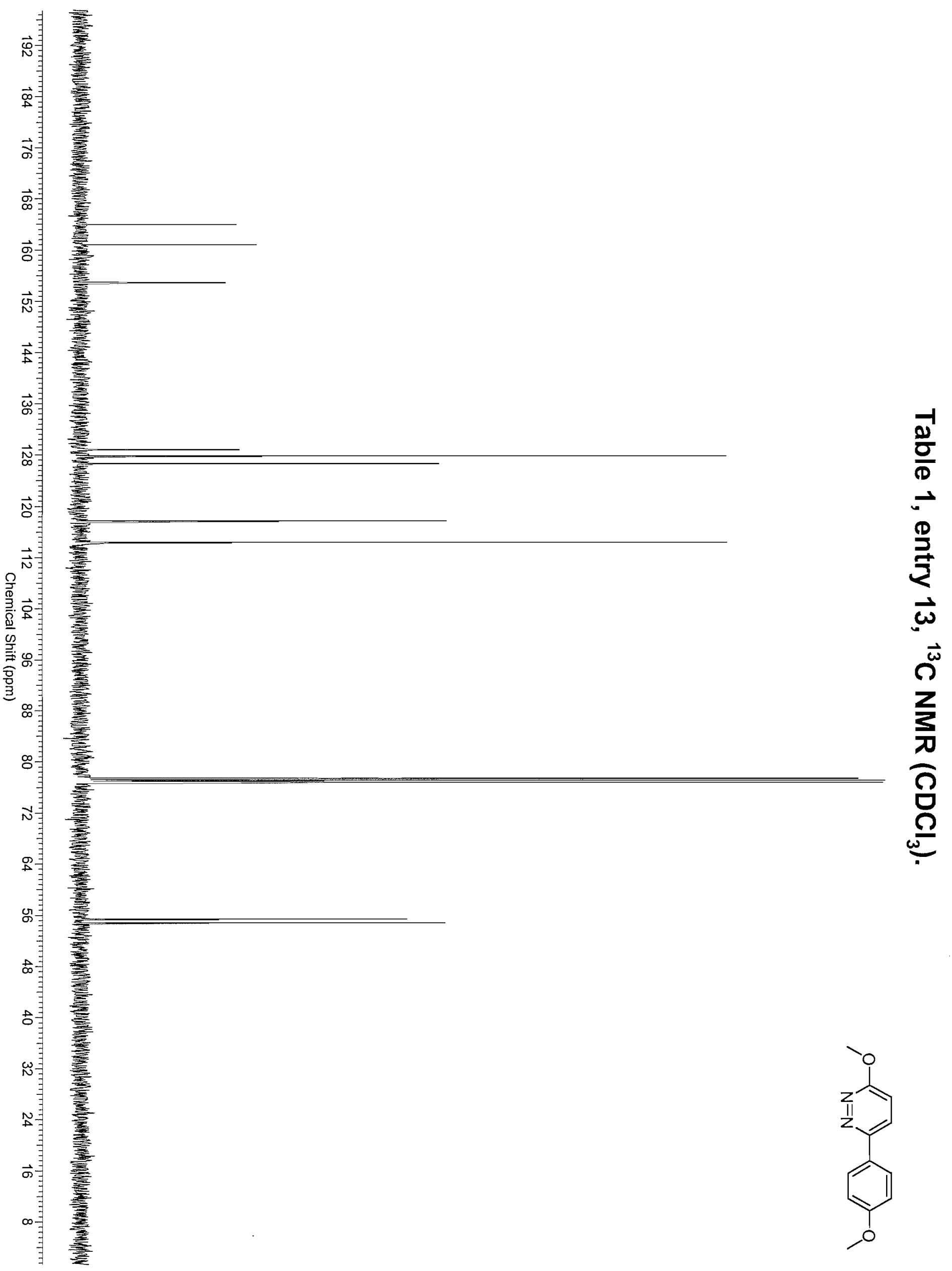




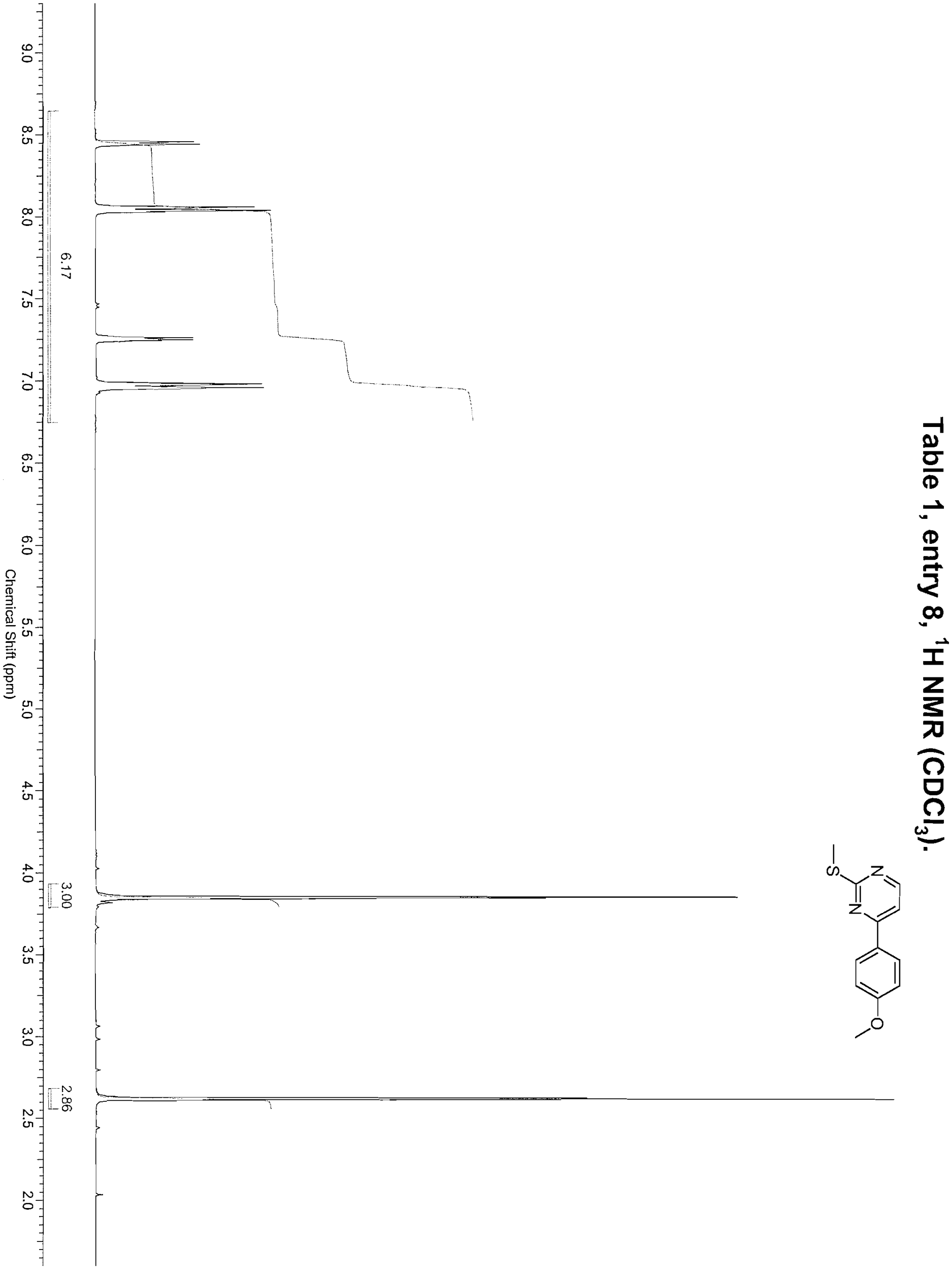




$$
E
$$




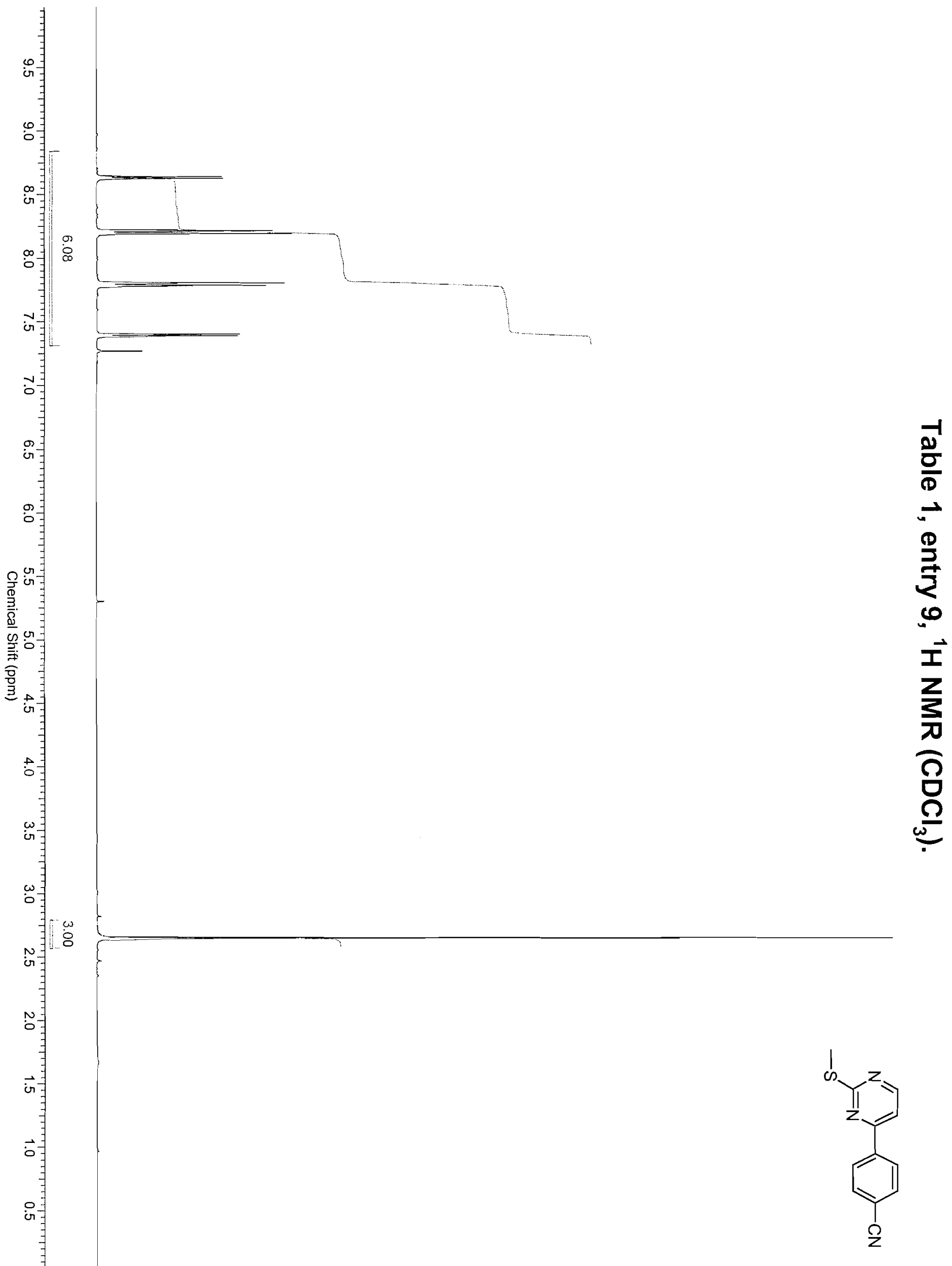




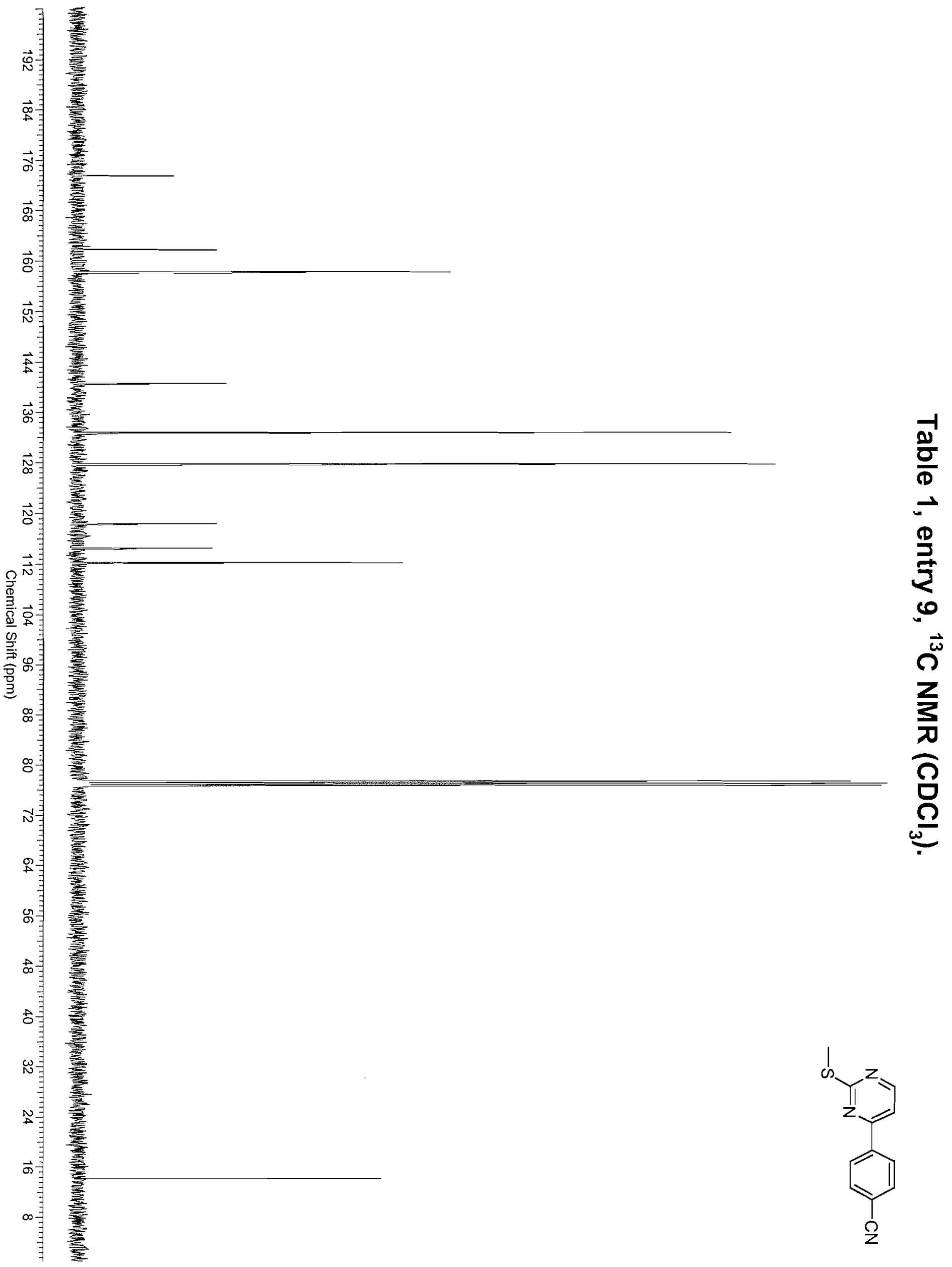




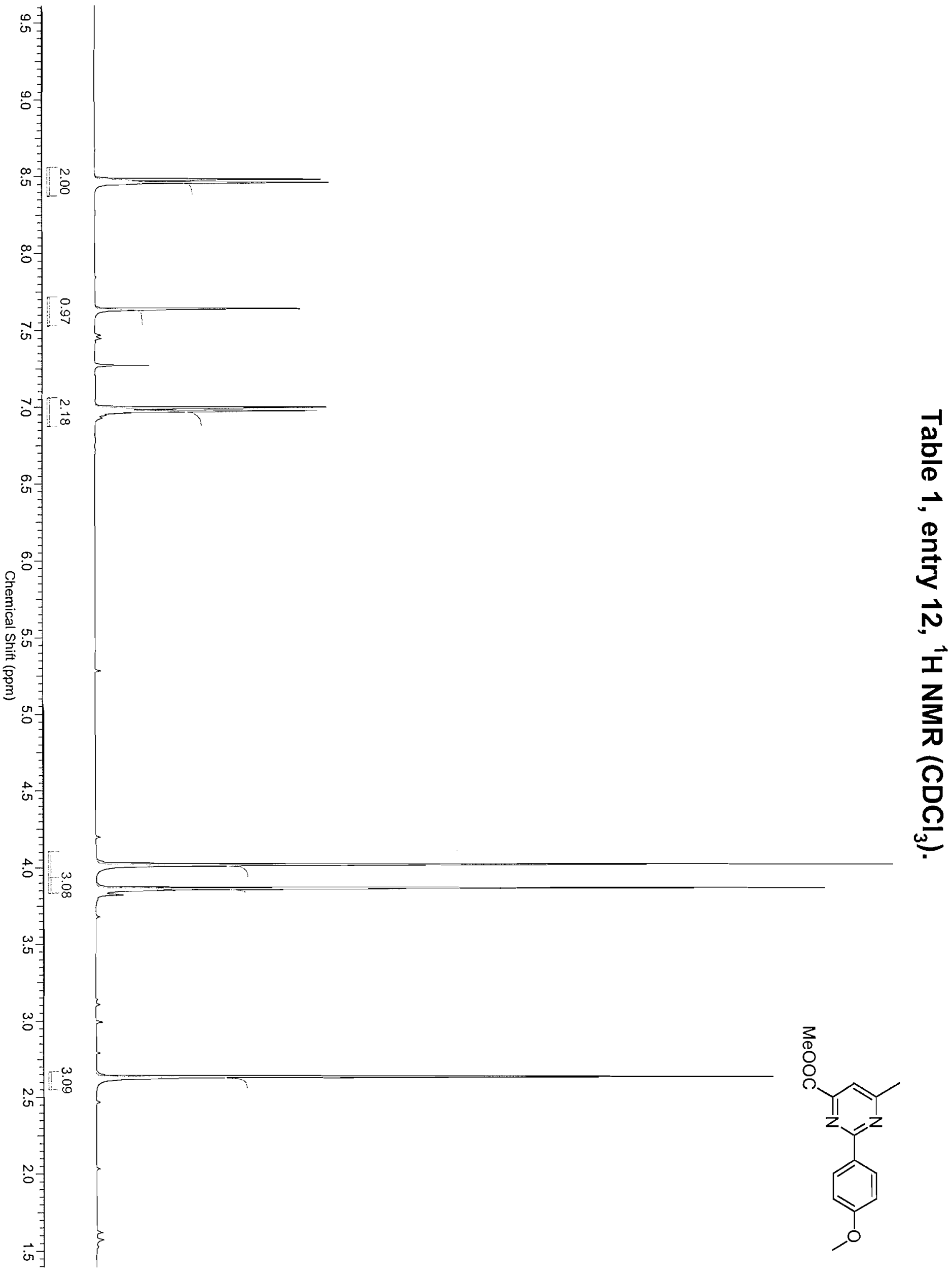




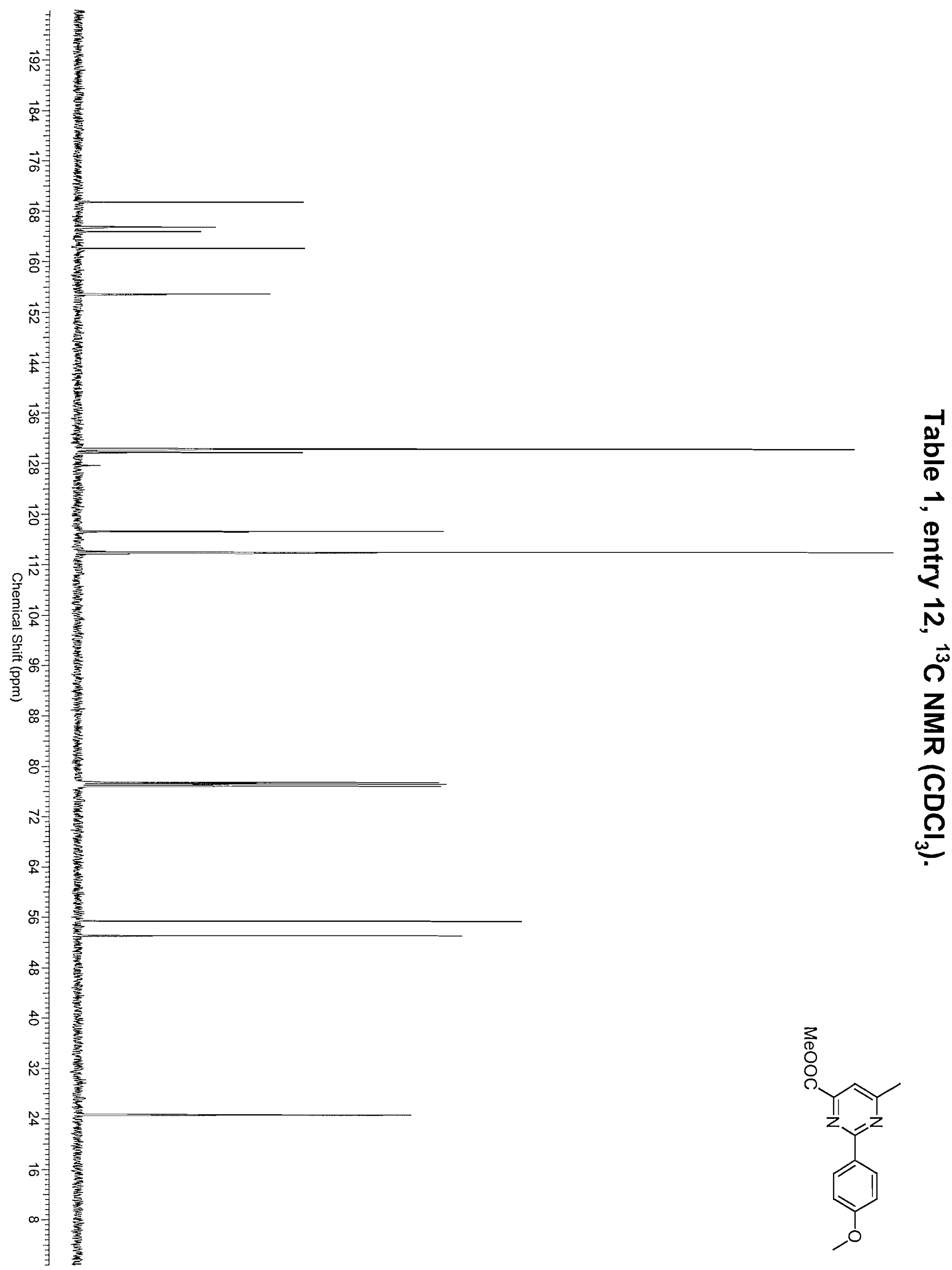




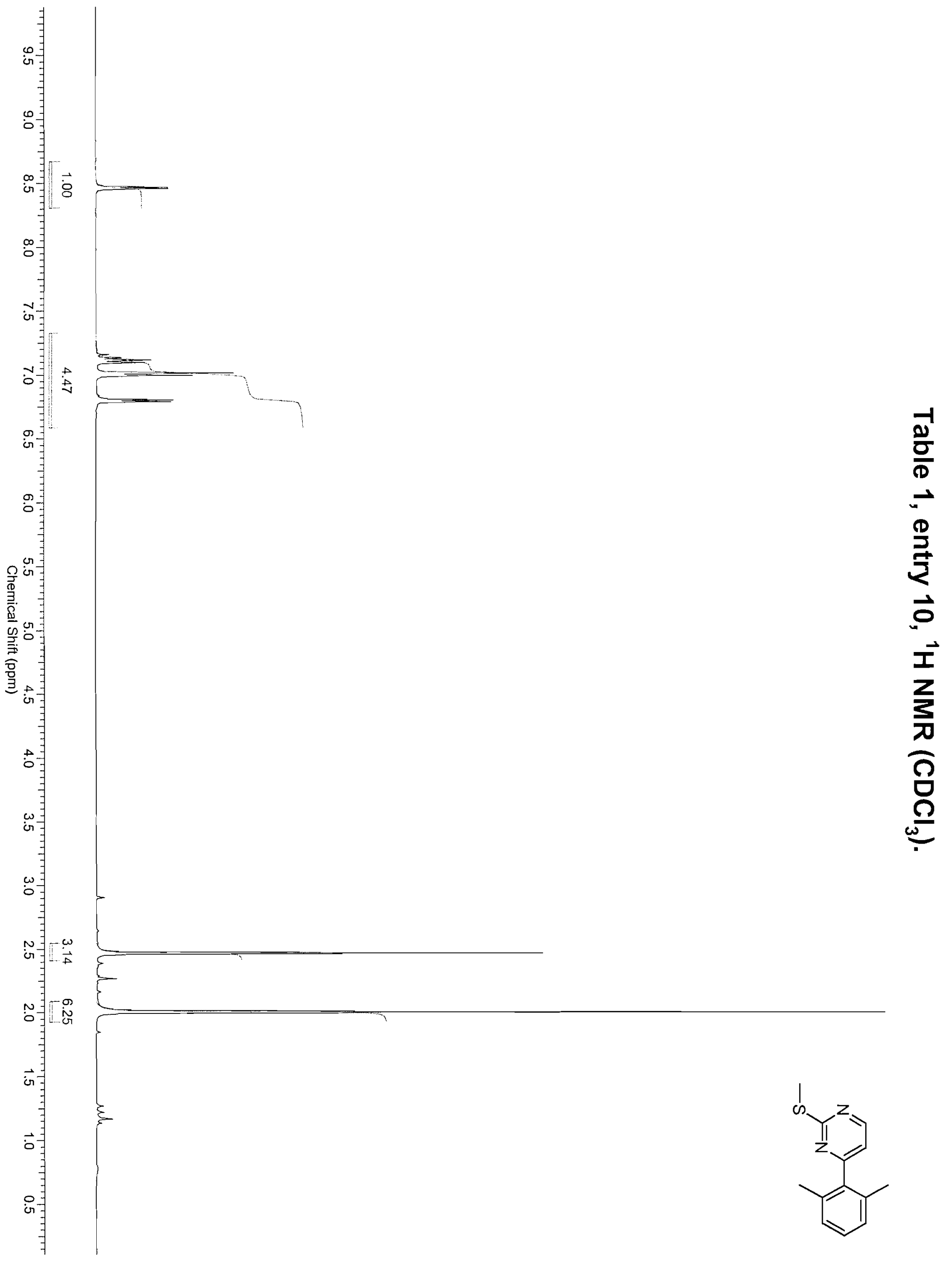


48

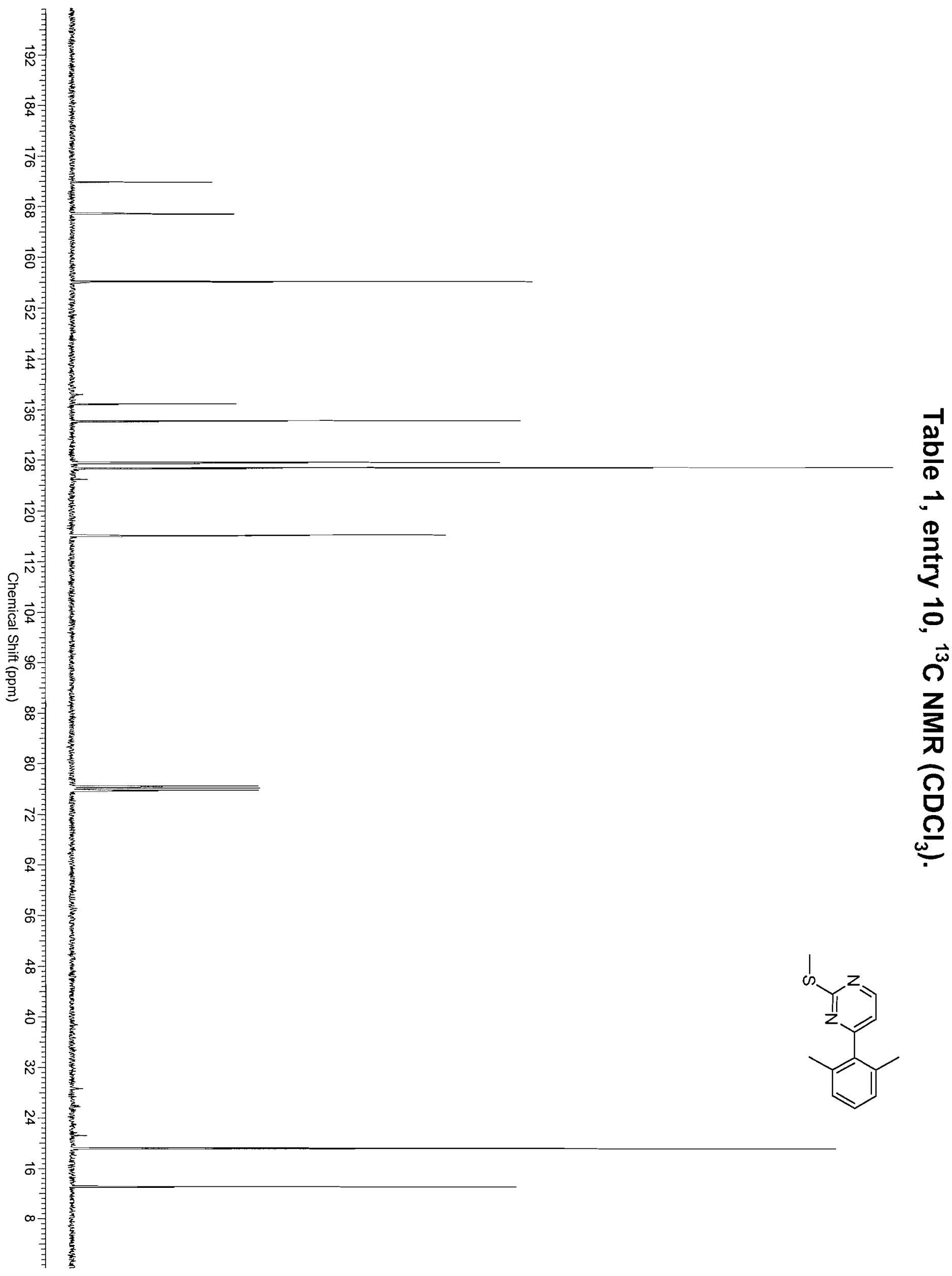




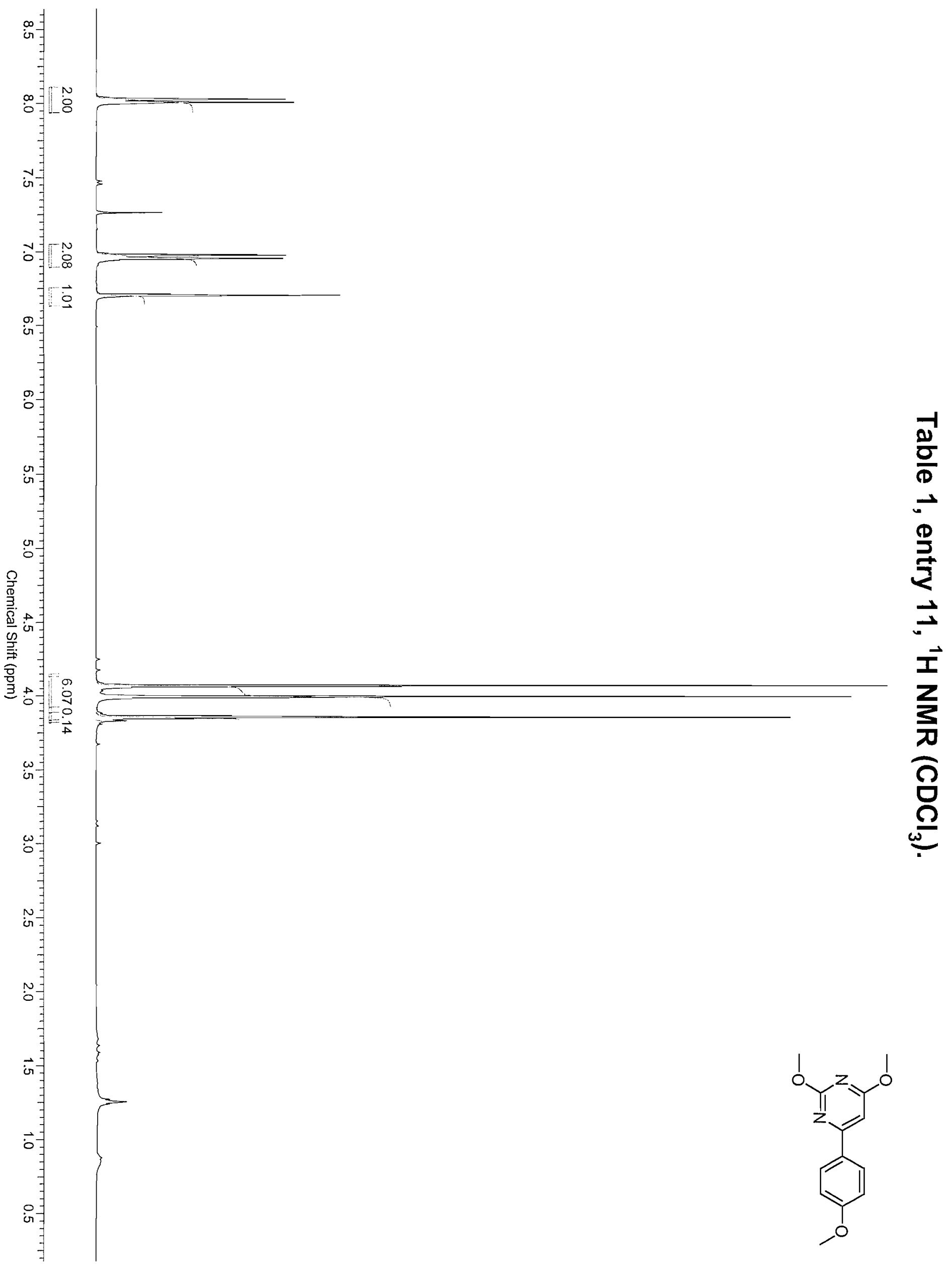




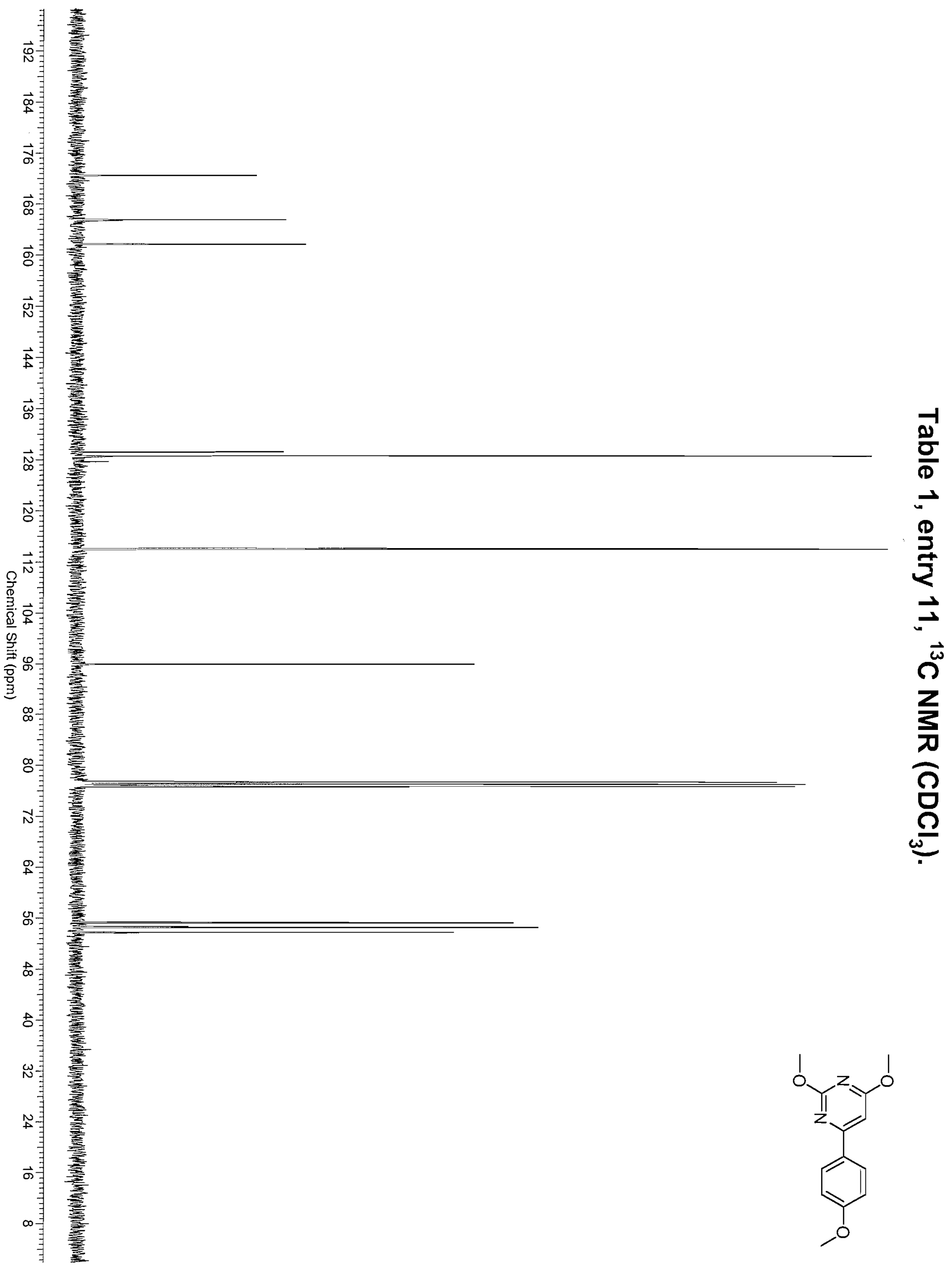




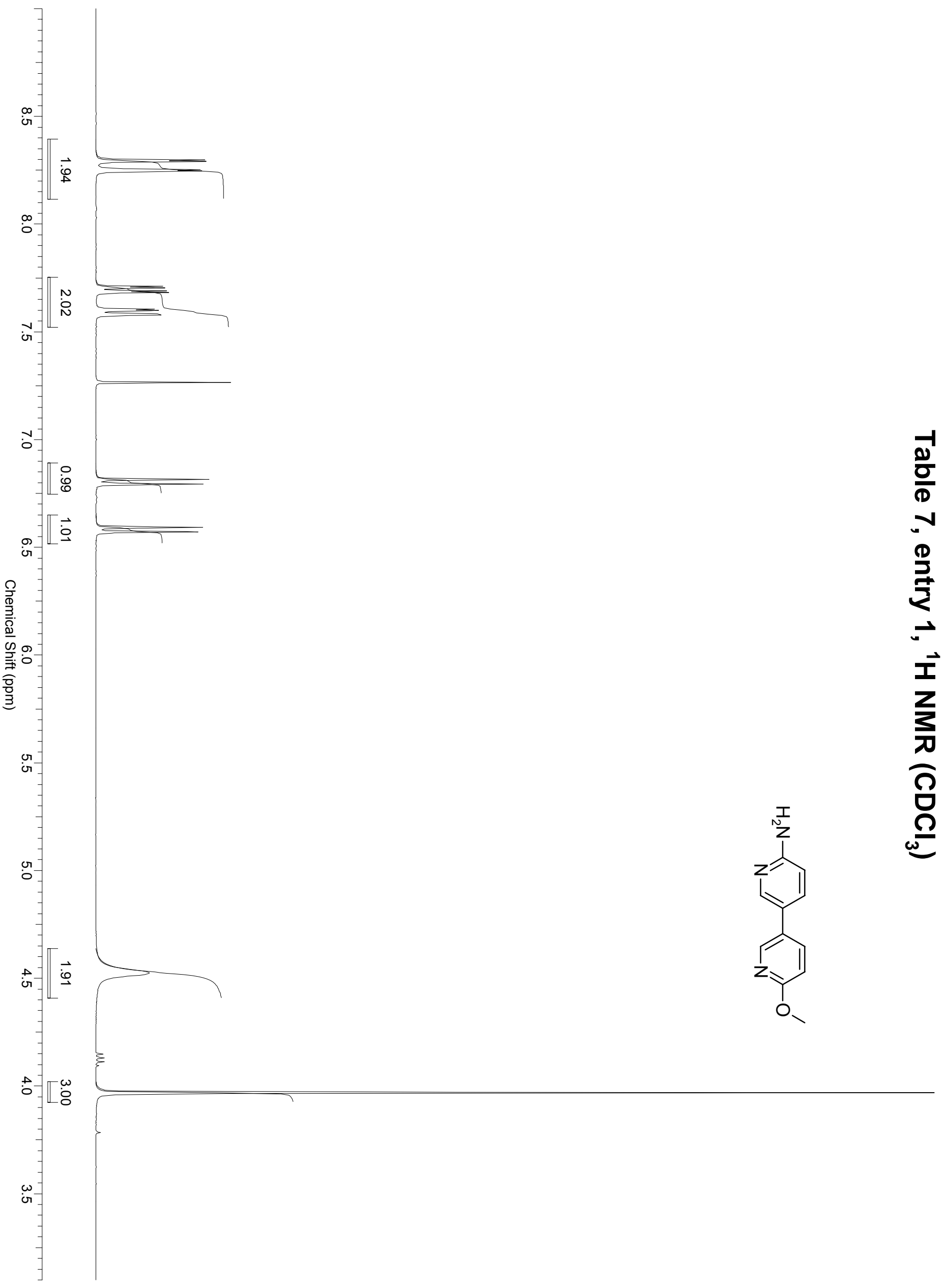




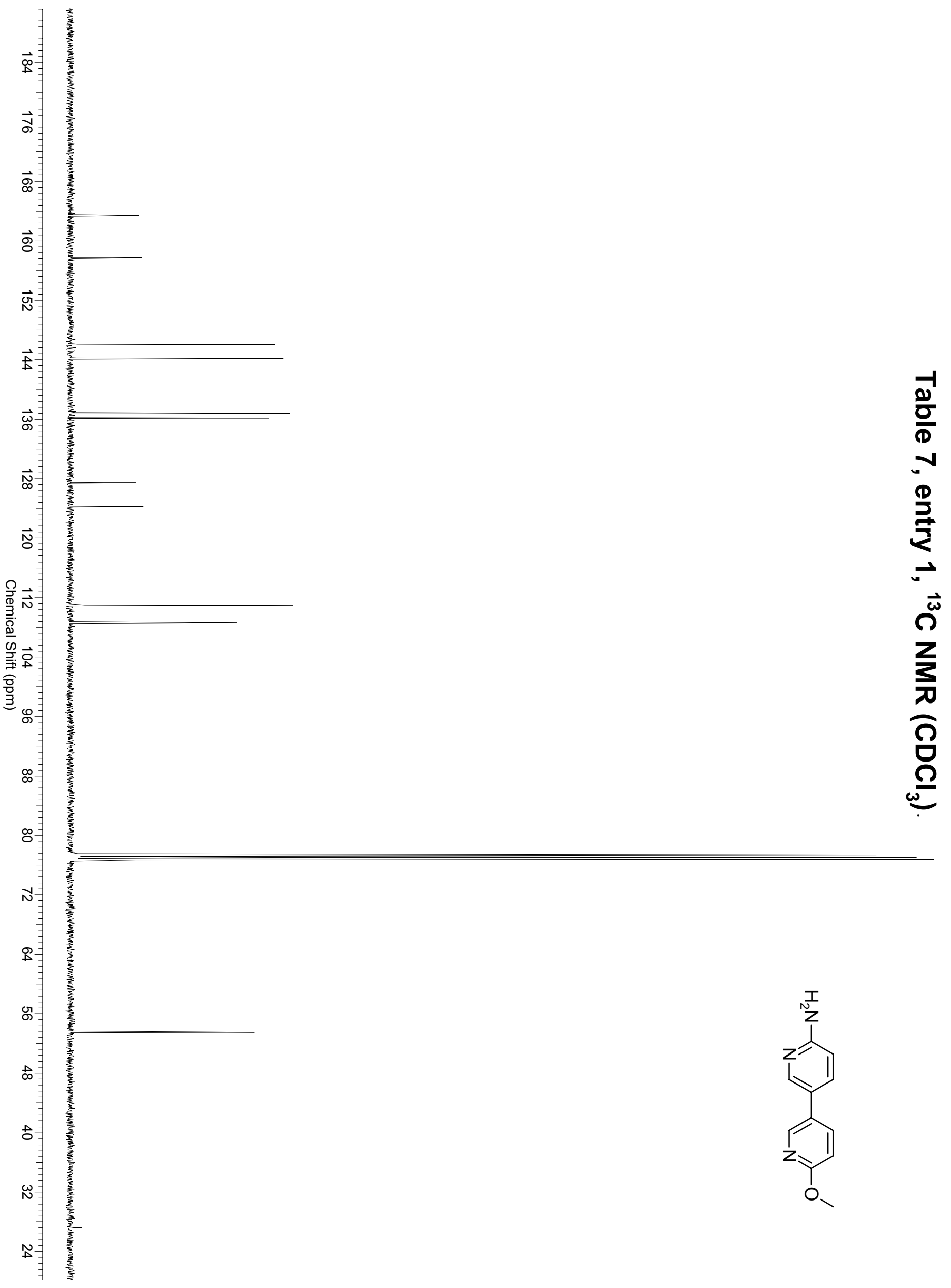




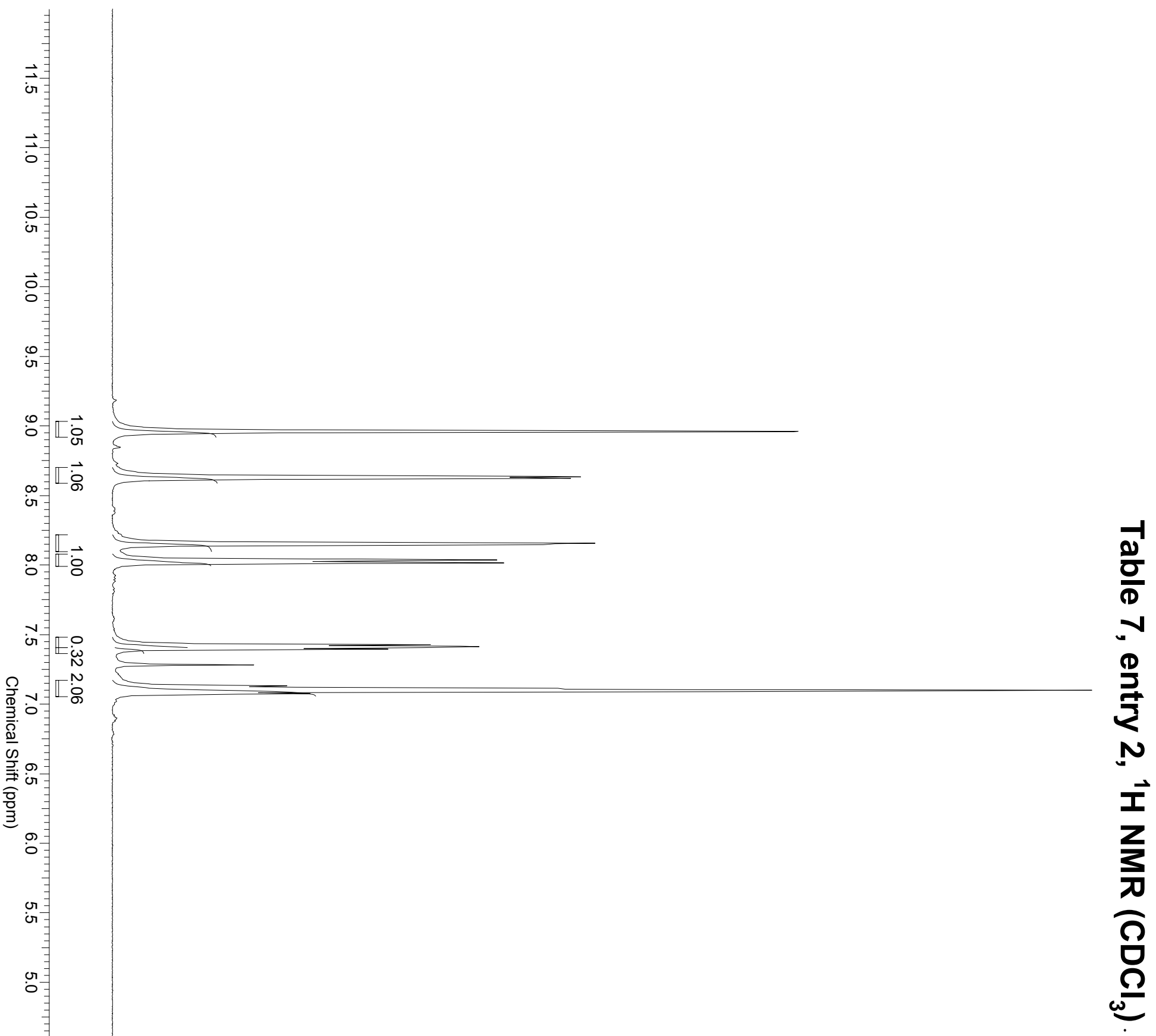

iि

当 $N$

$\omega$

$\omega$

0

i

o

N

نे 


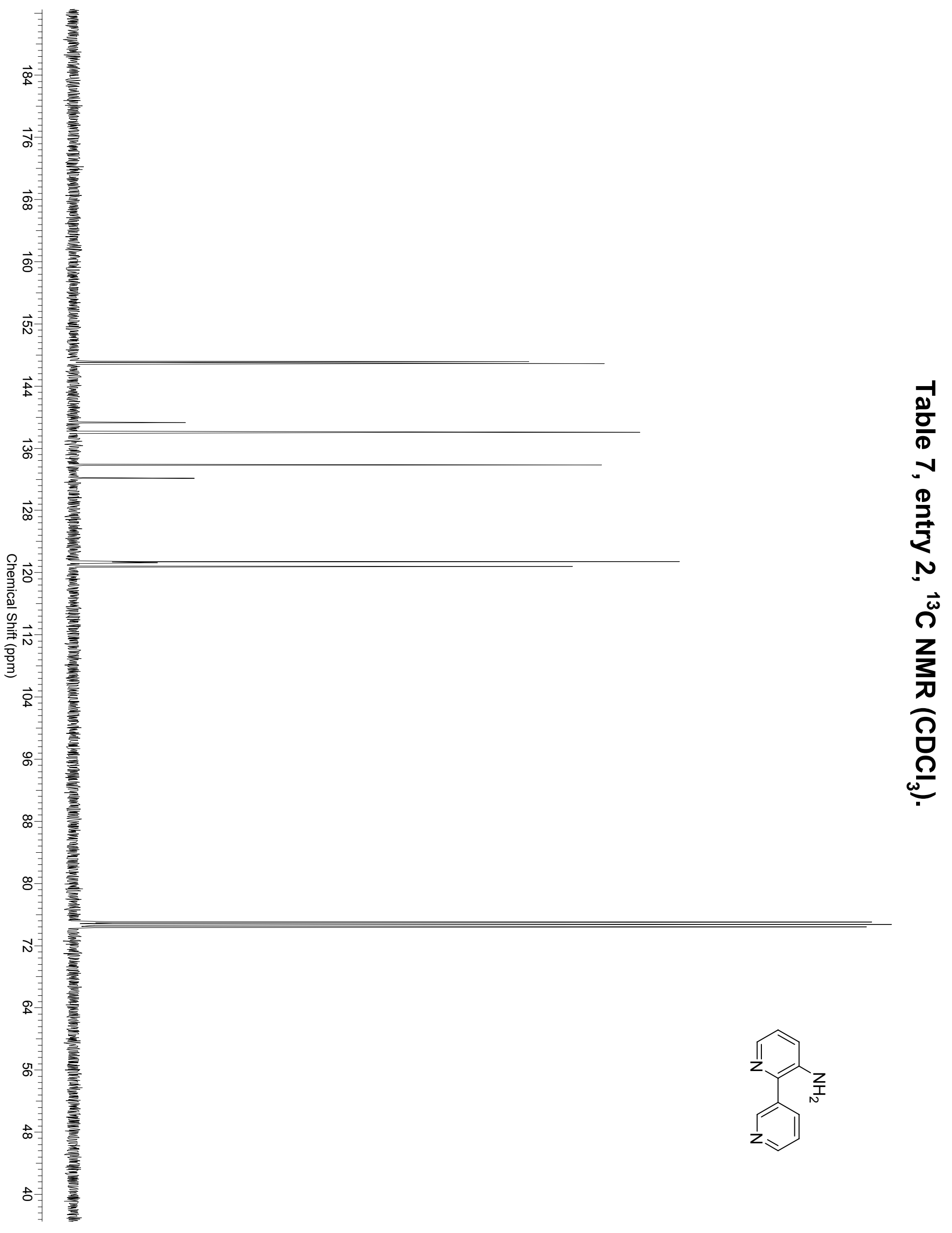



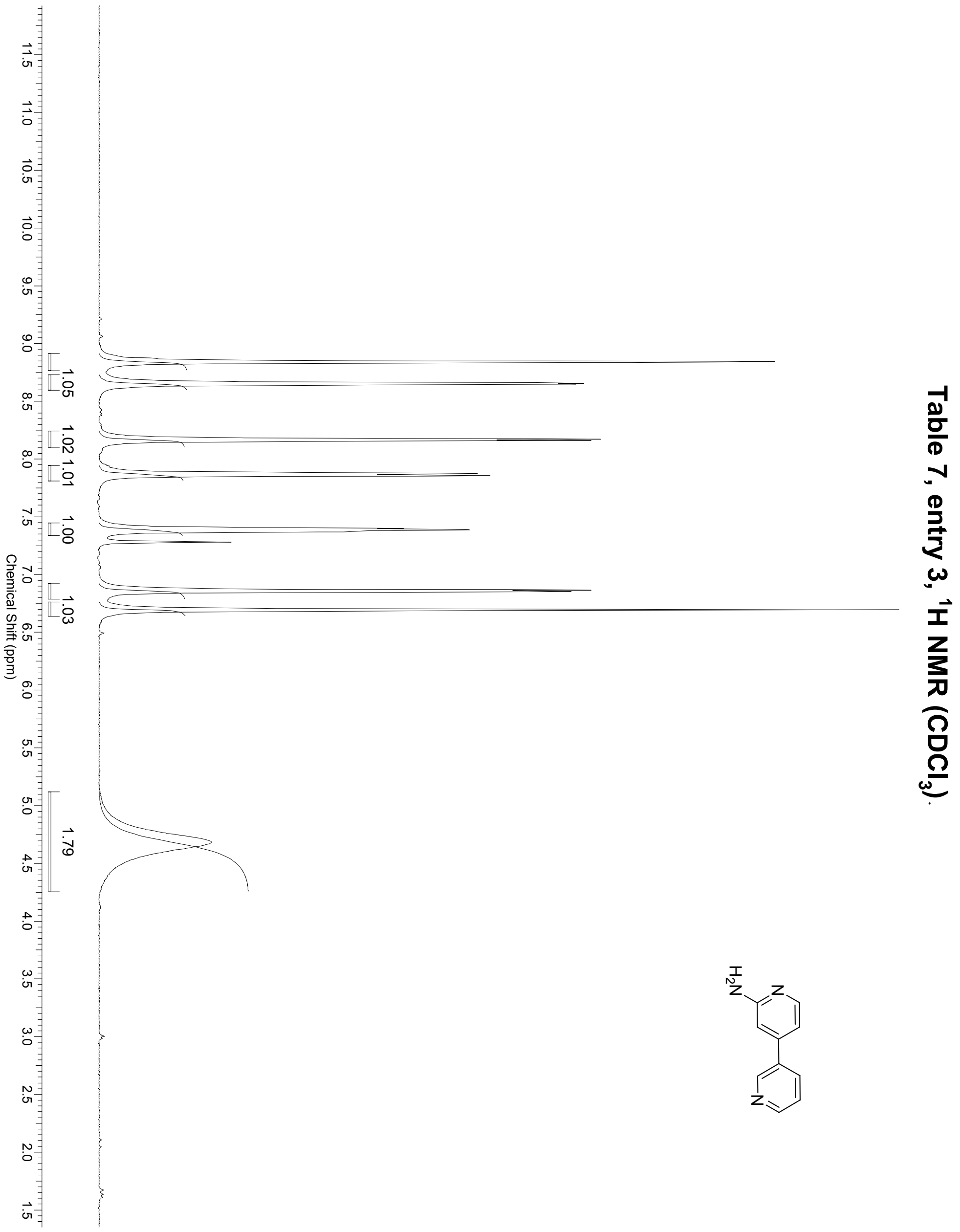


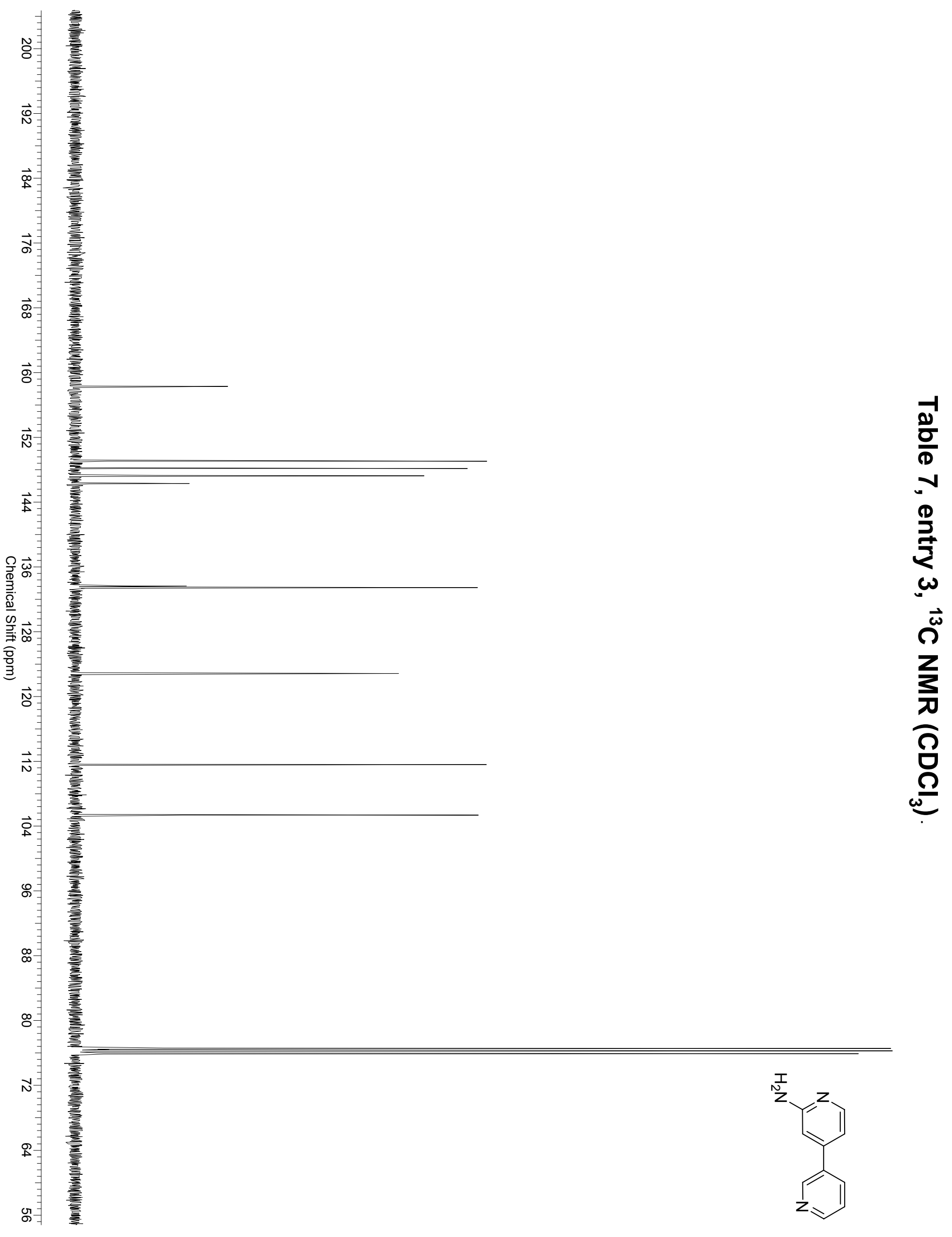




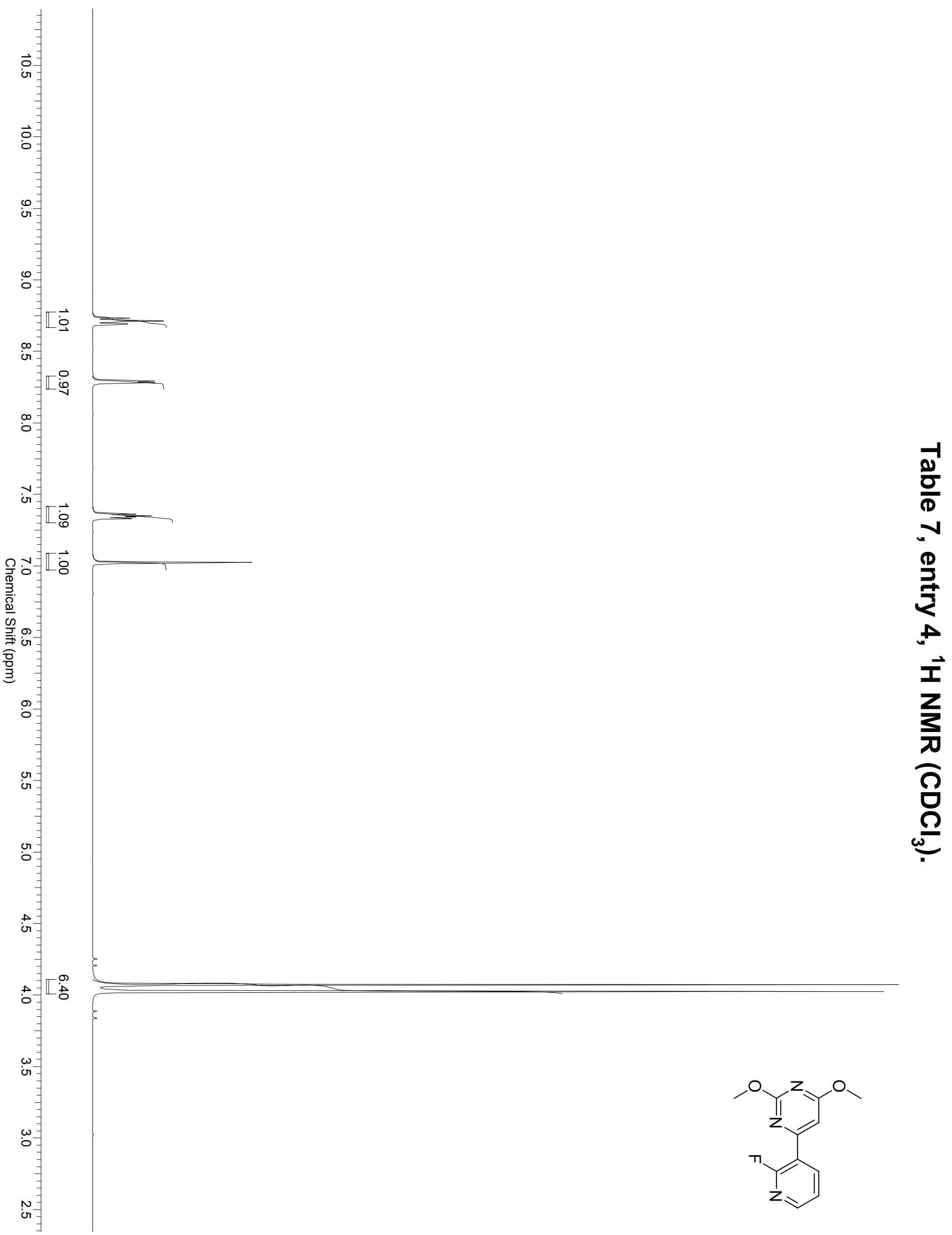




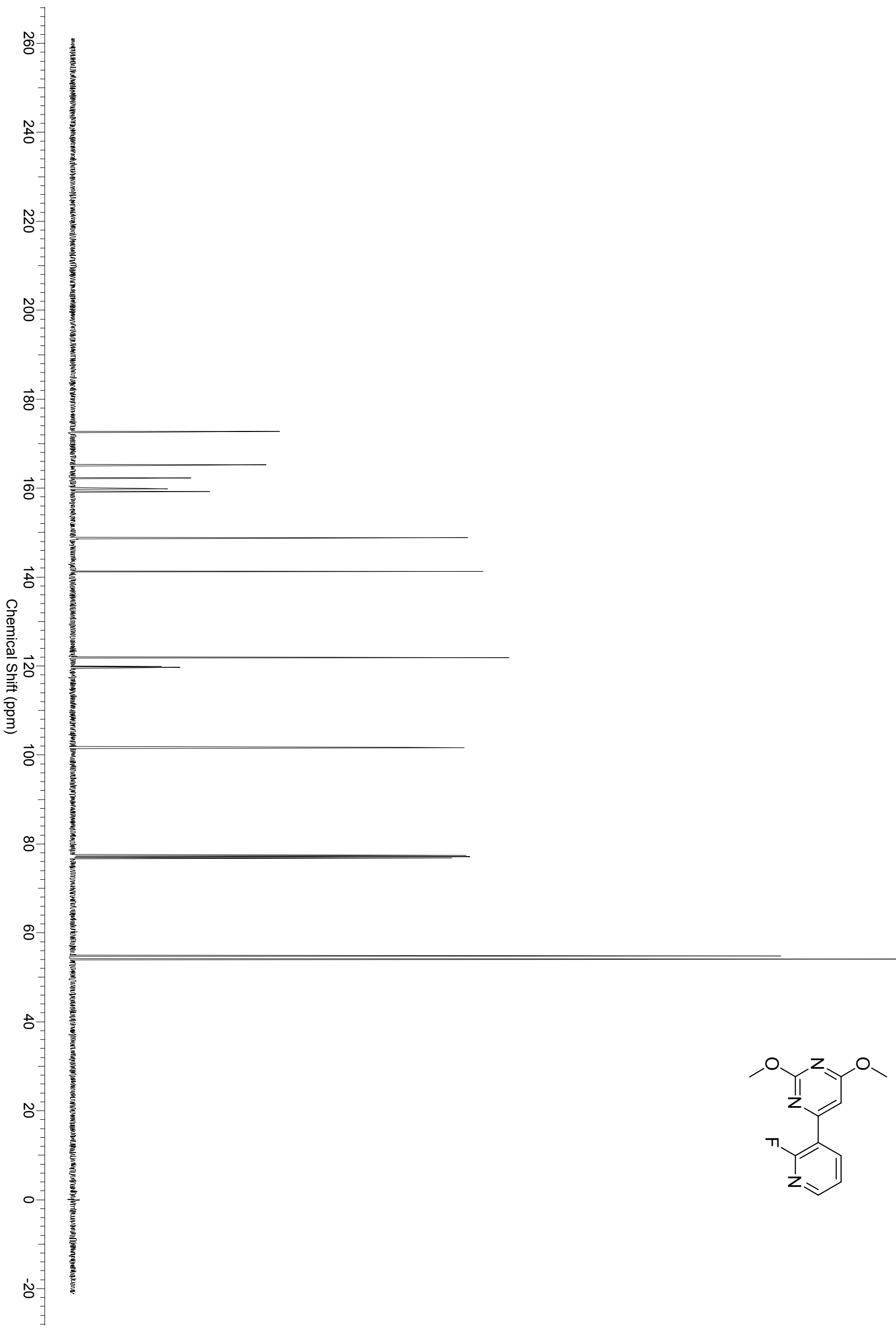

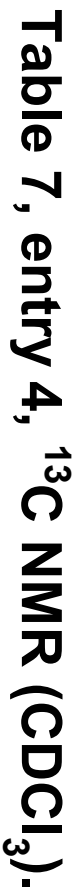



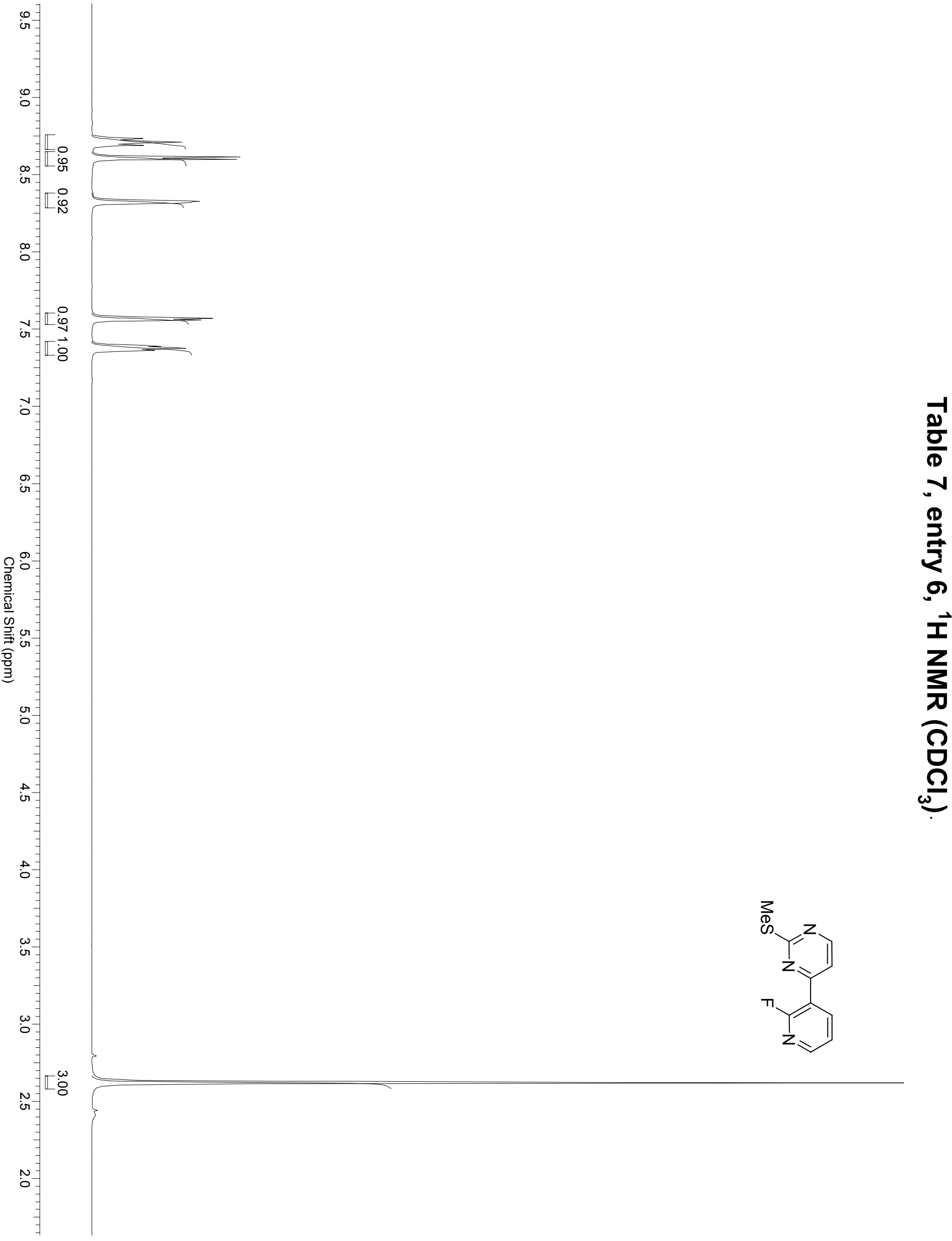

룰

ㅇ)

$\frac{1}{2}$

ก

อ

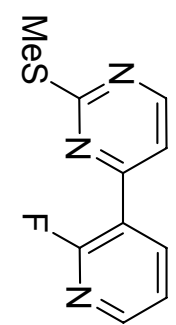




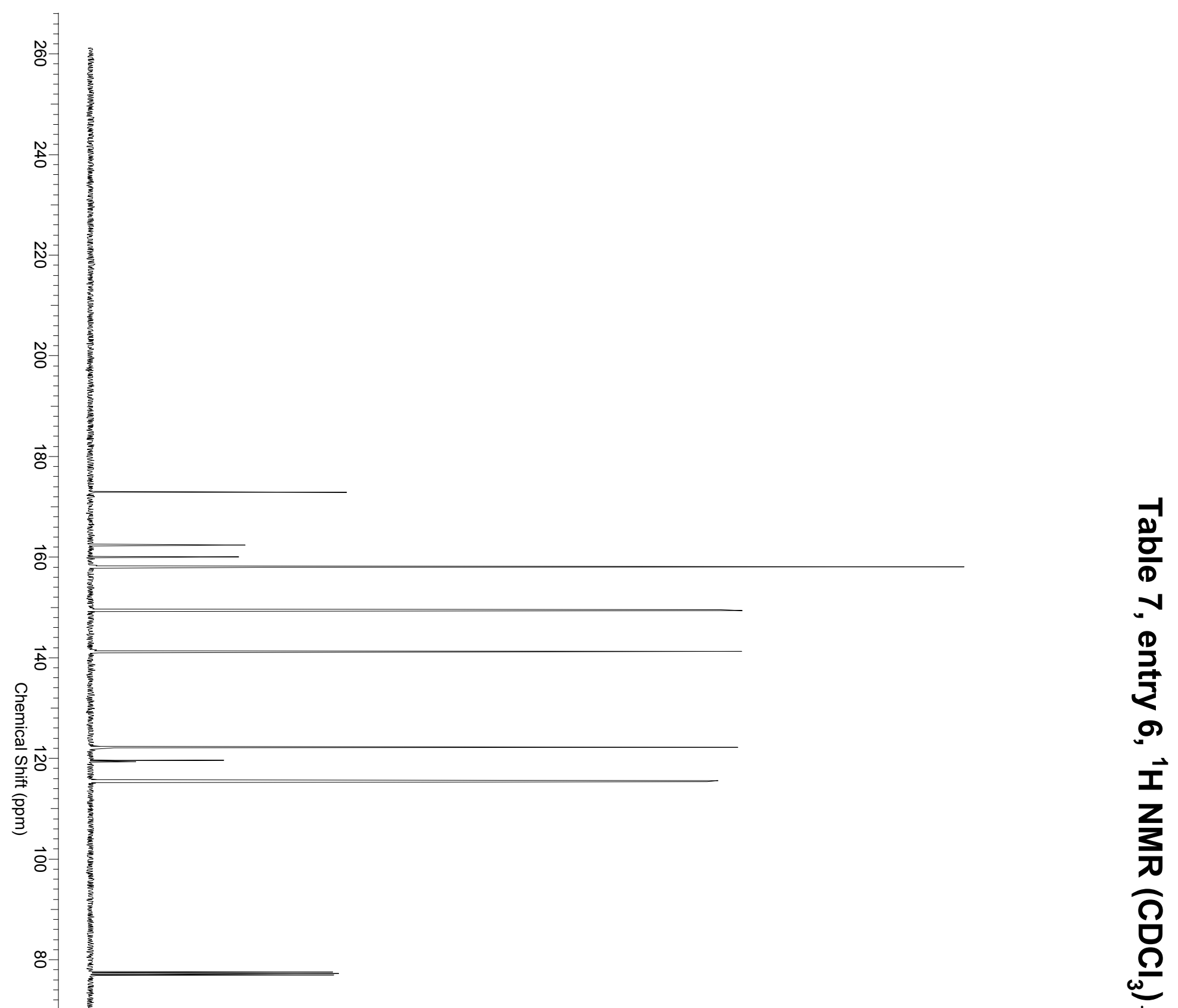

$8-$

o

음

N

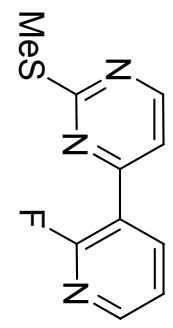




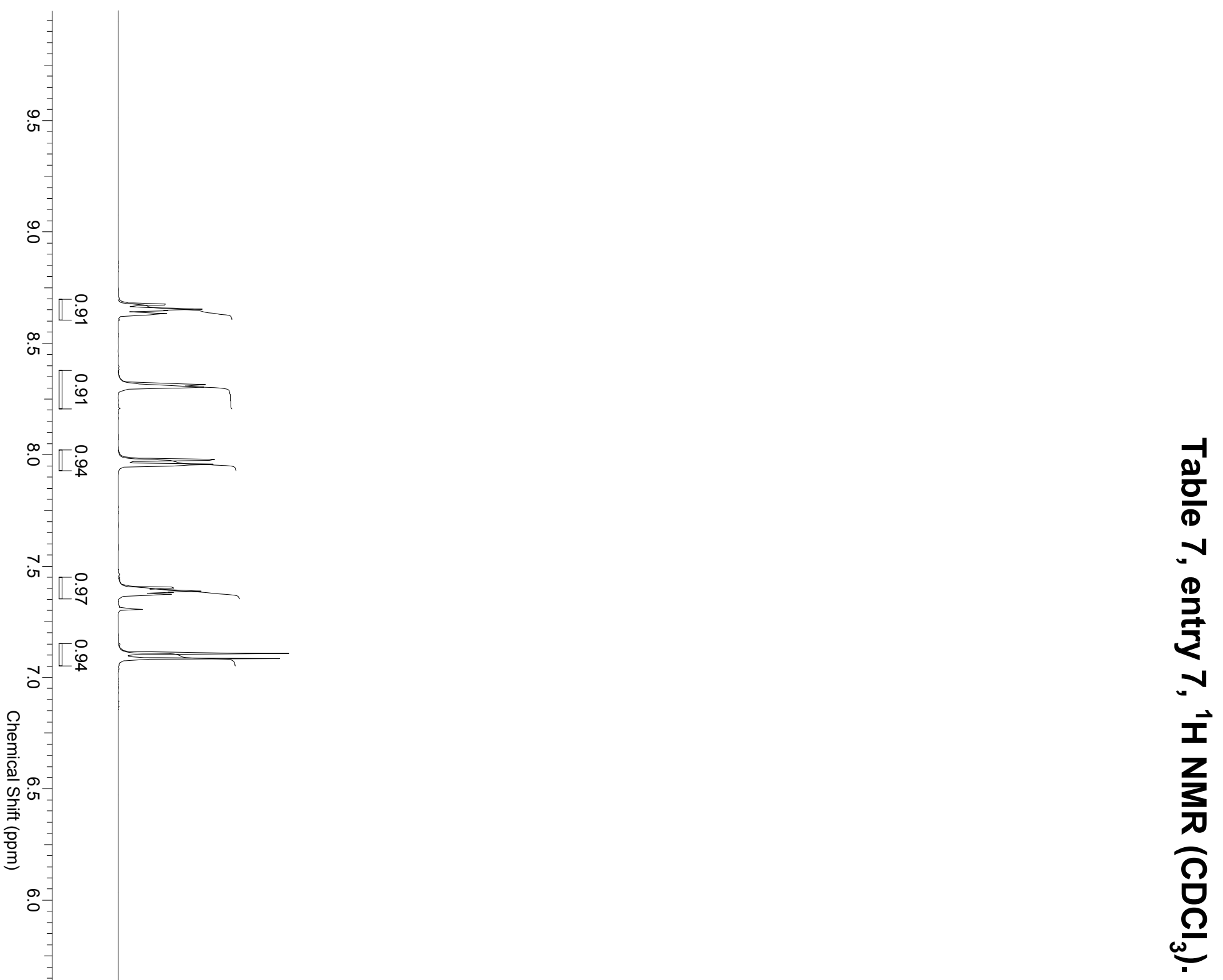

or

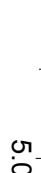

or

ir

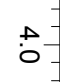

$\omega$
8

$\omega$

$\omega=$

$\omega$

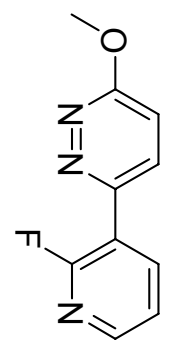




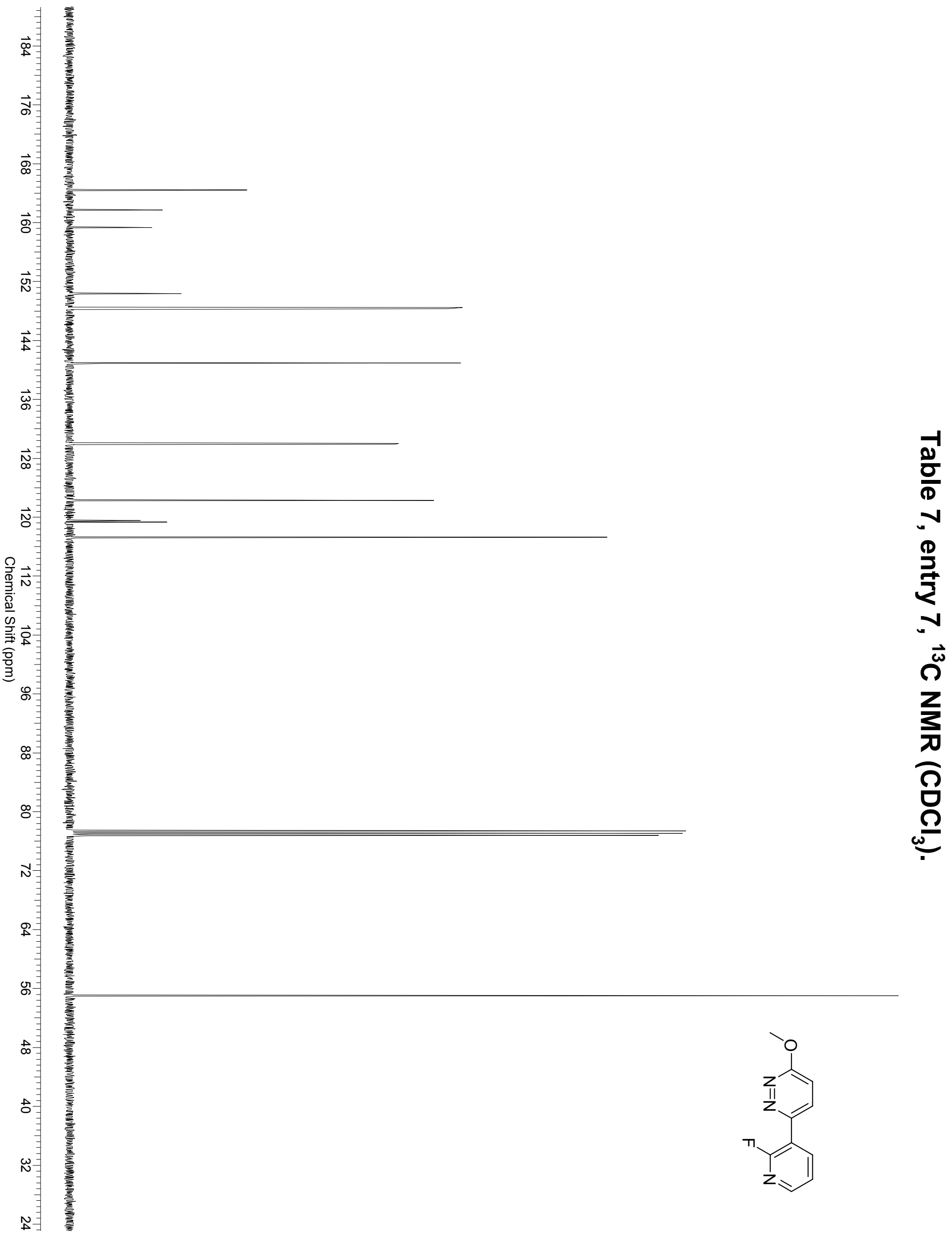



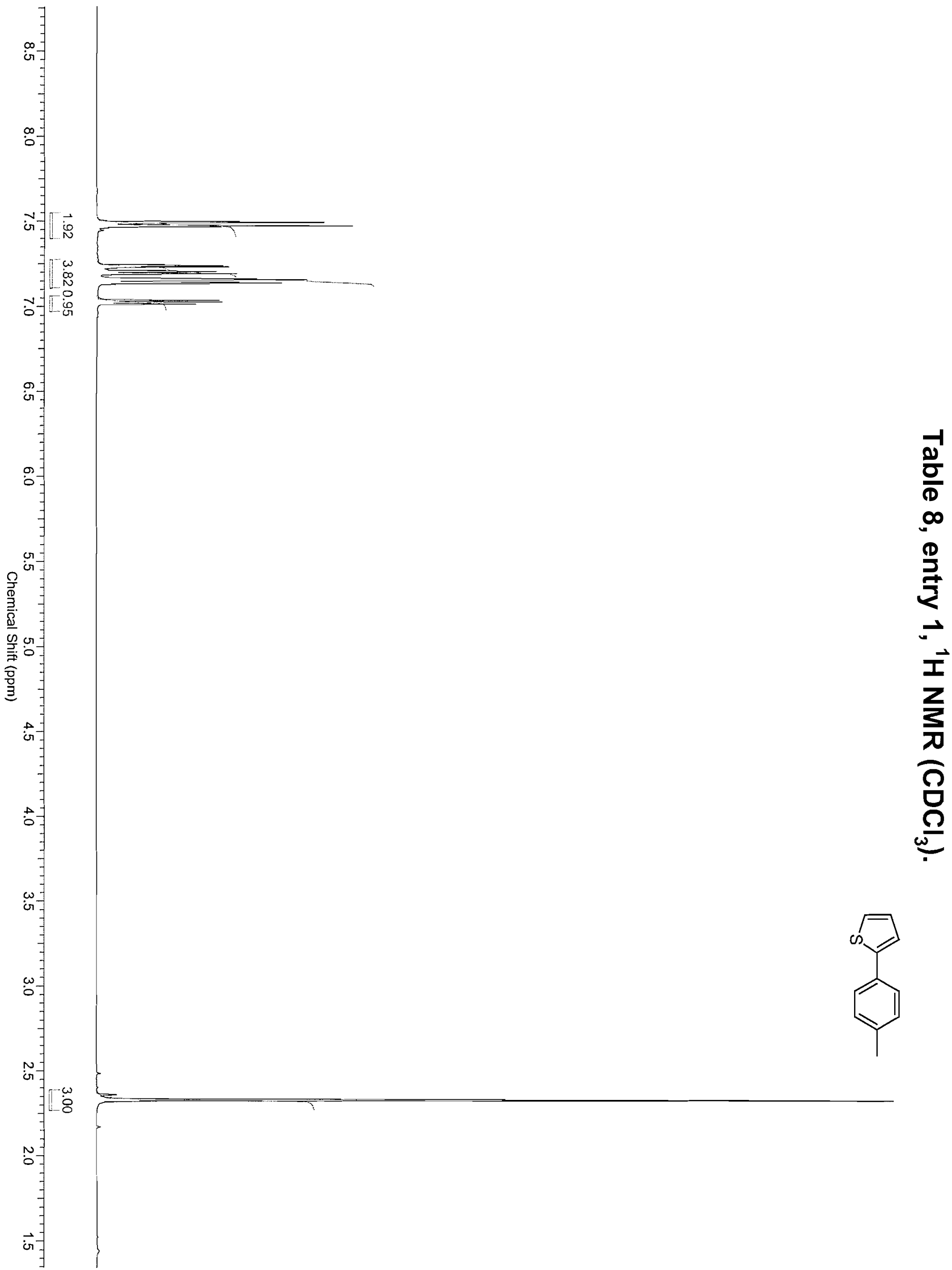


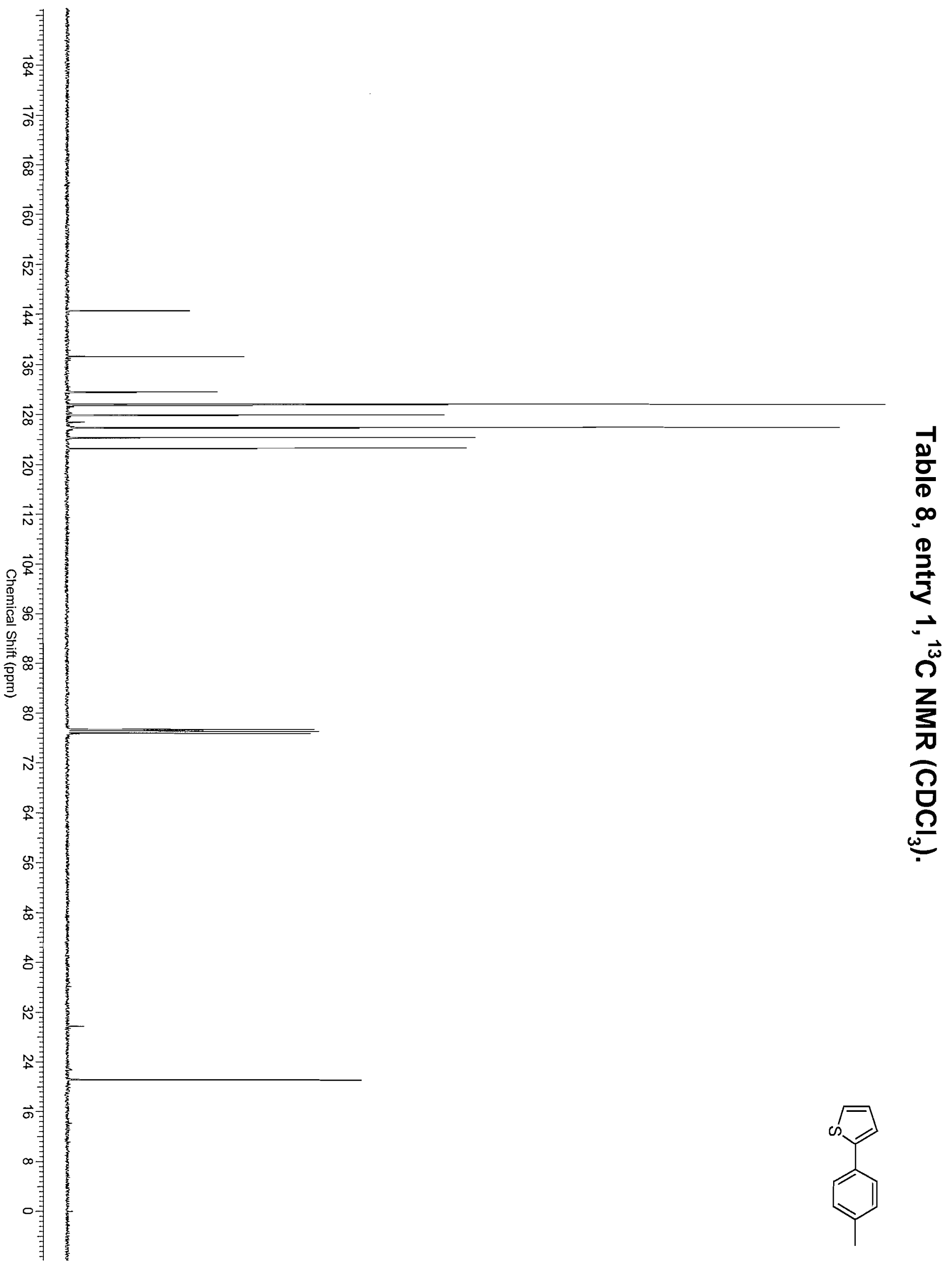




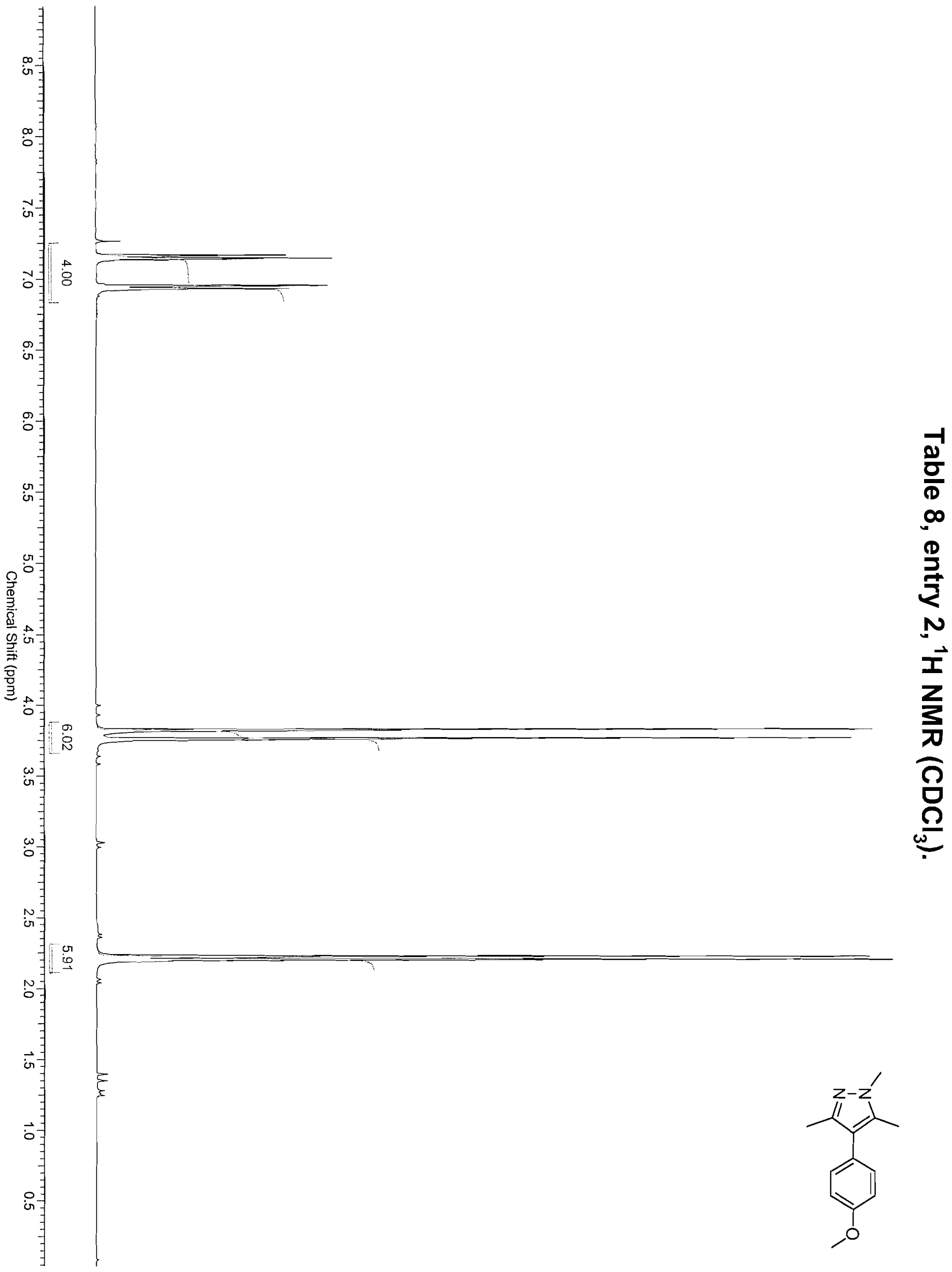




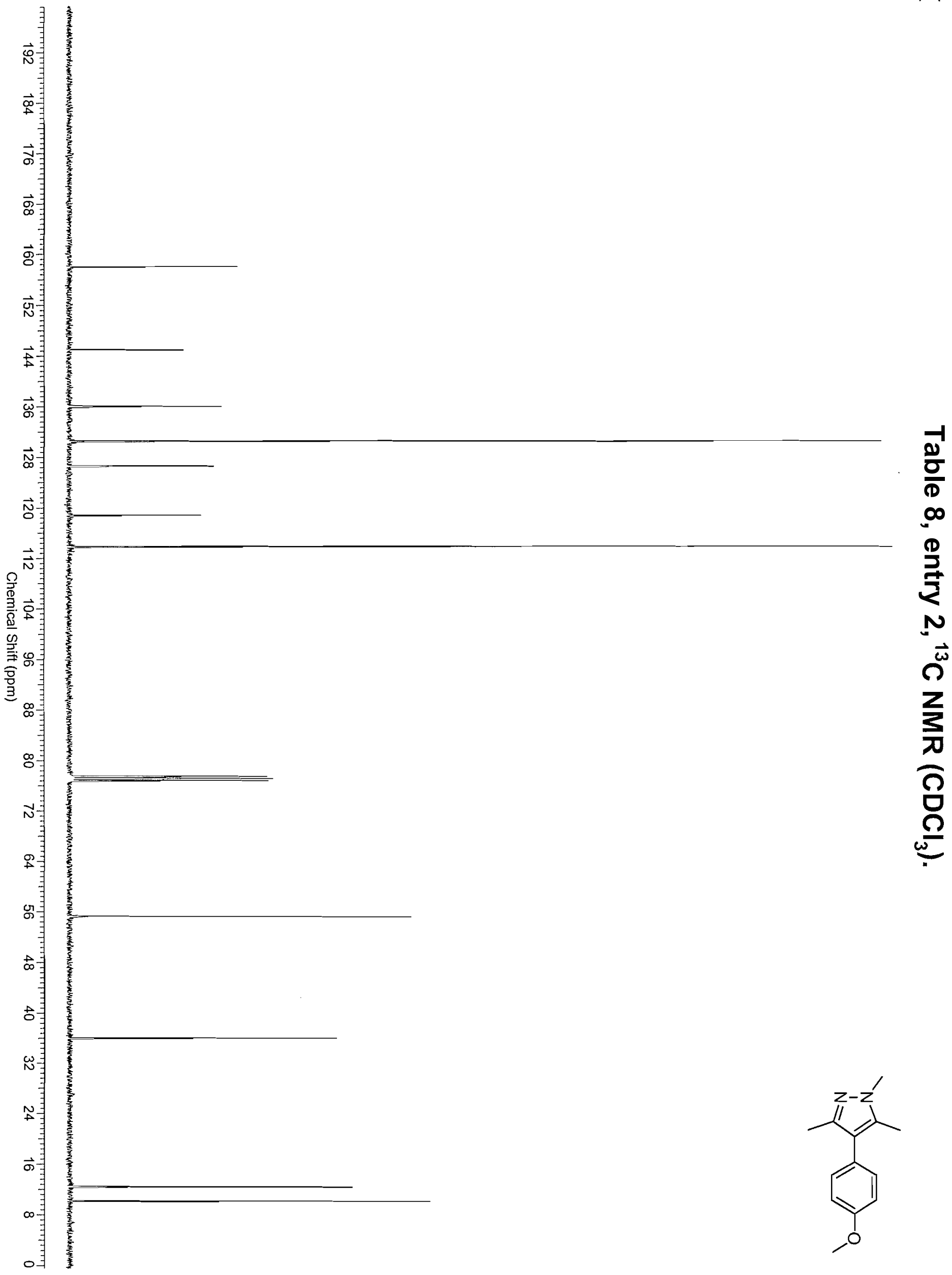




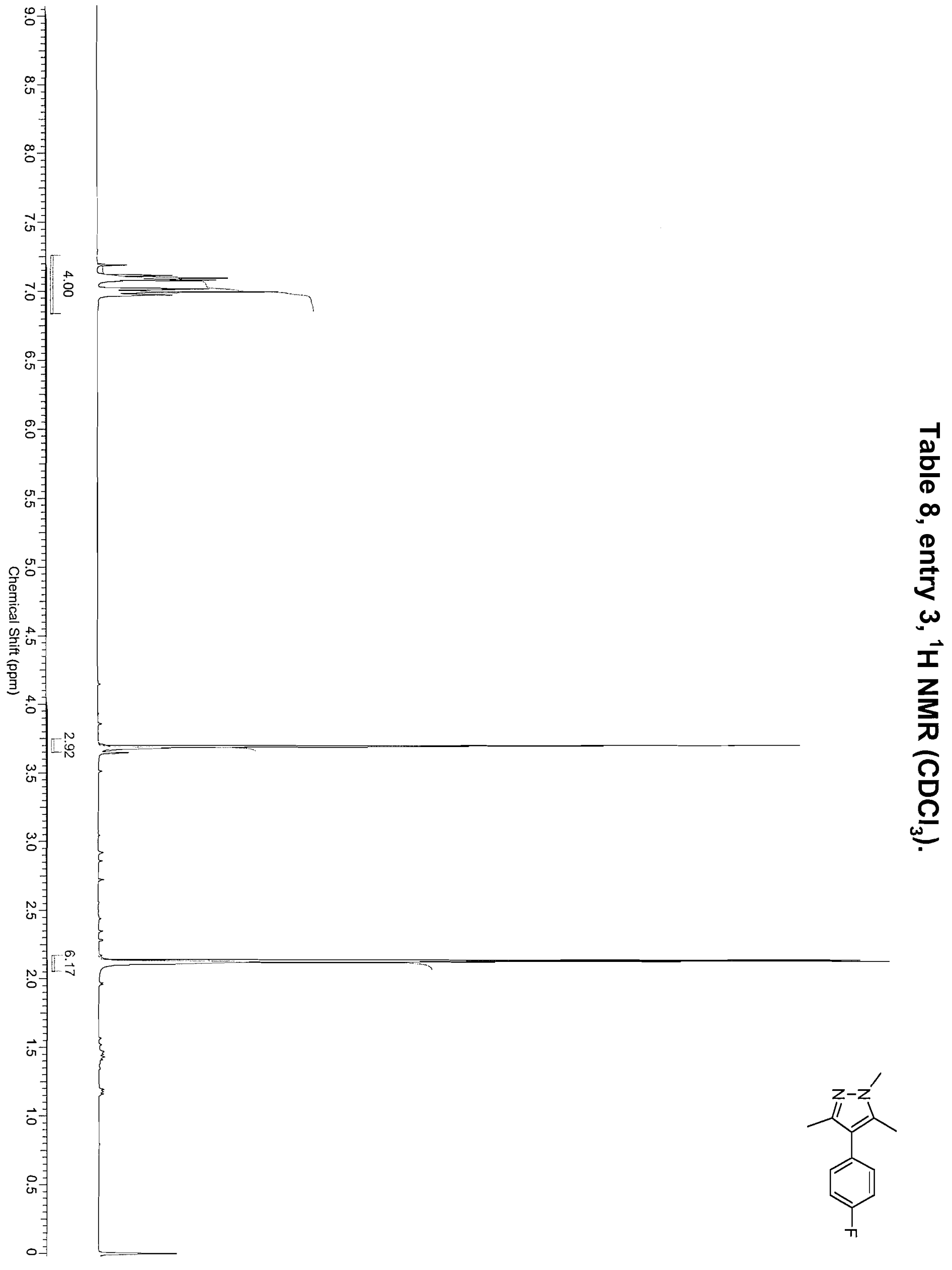



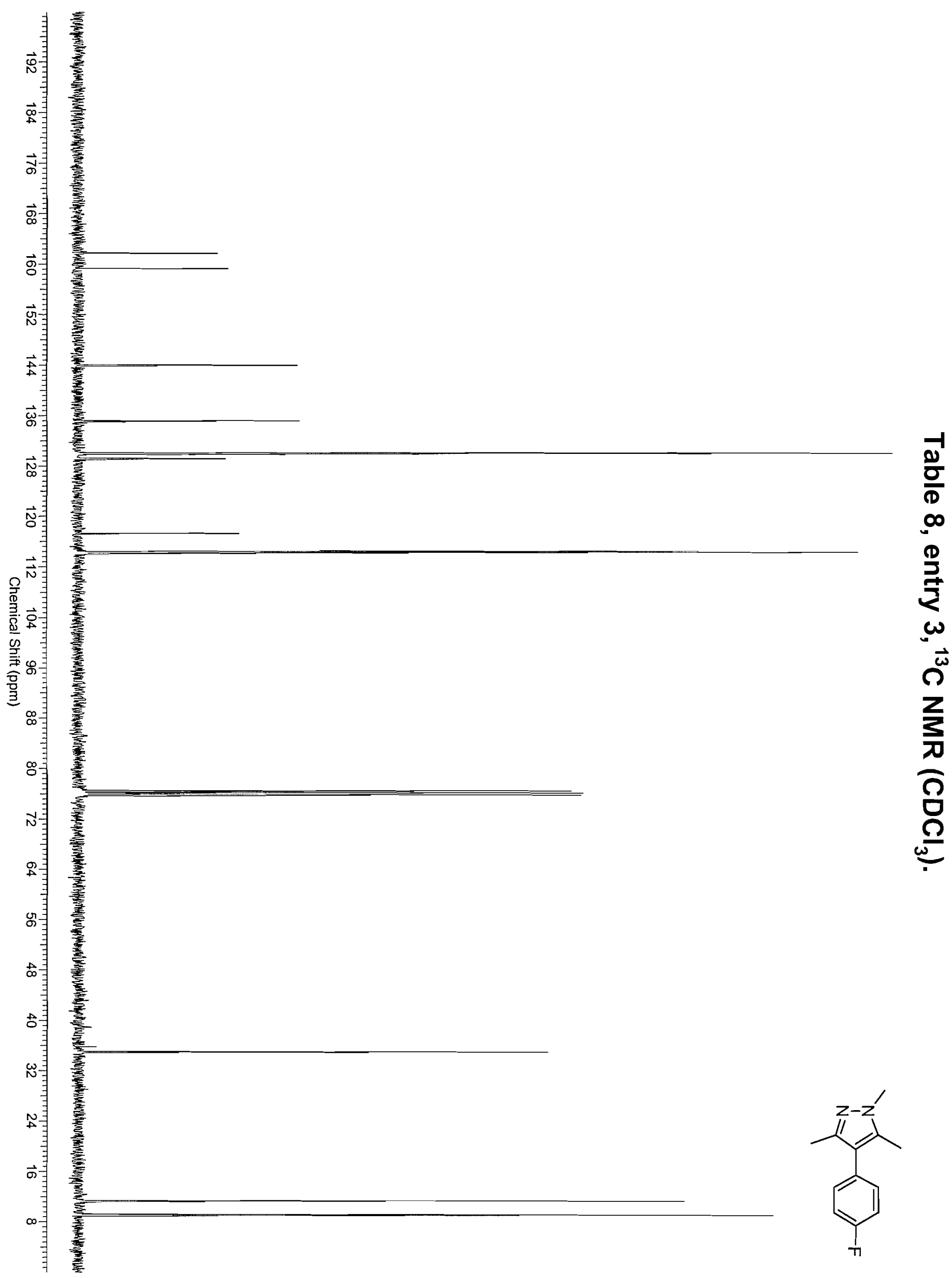


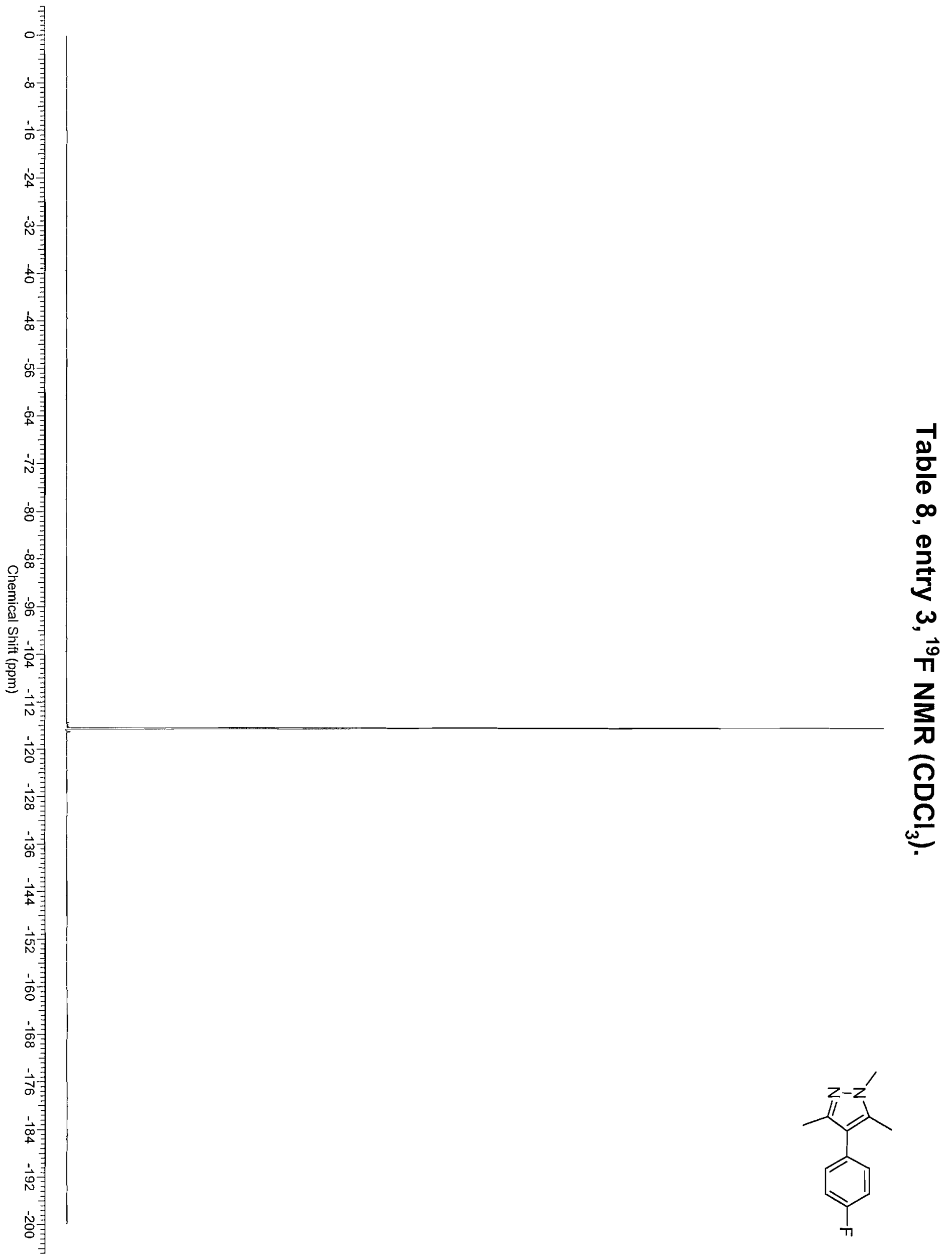




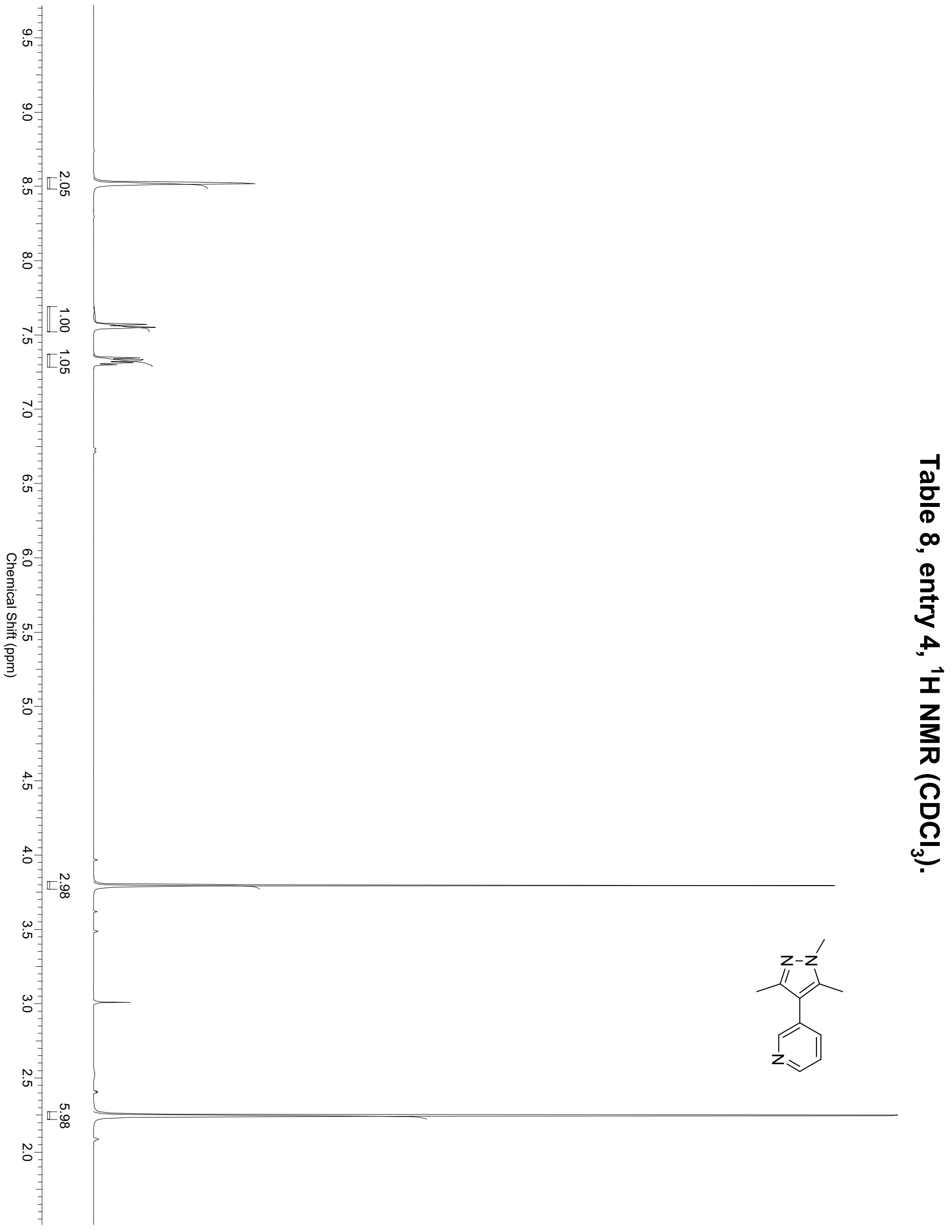



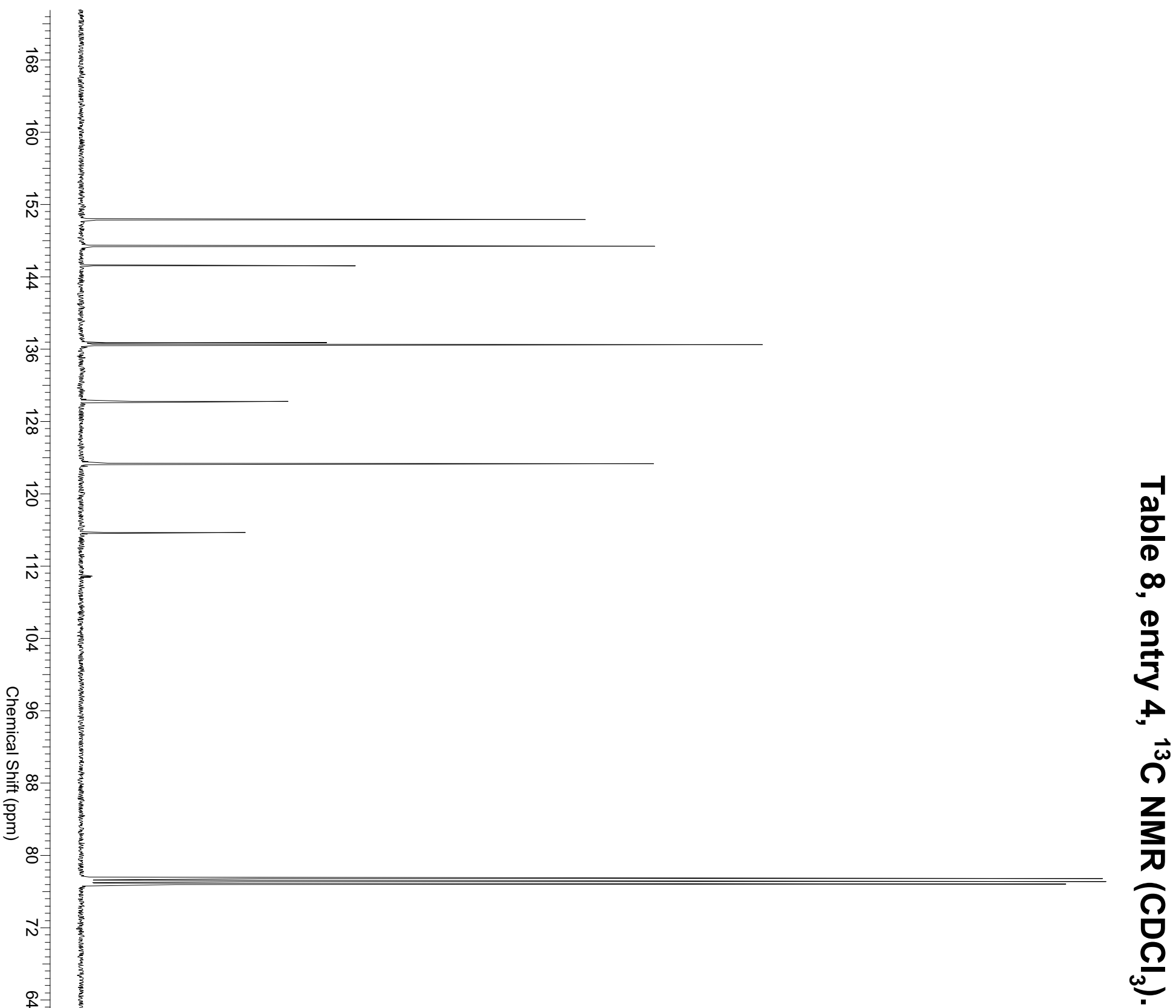

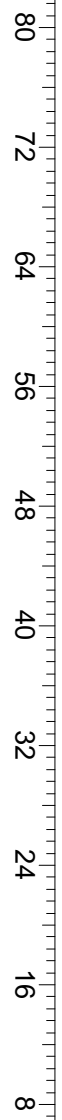




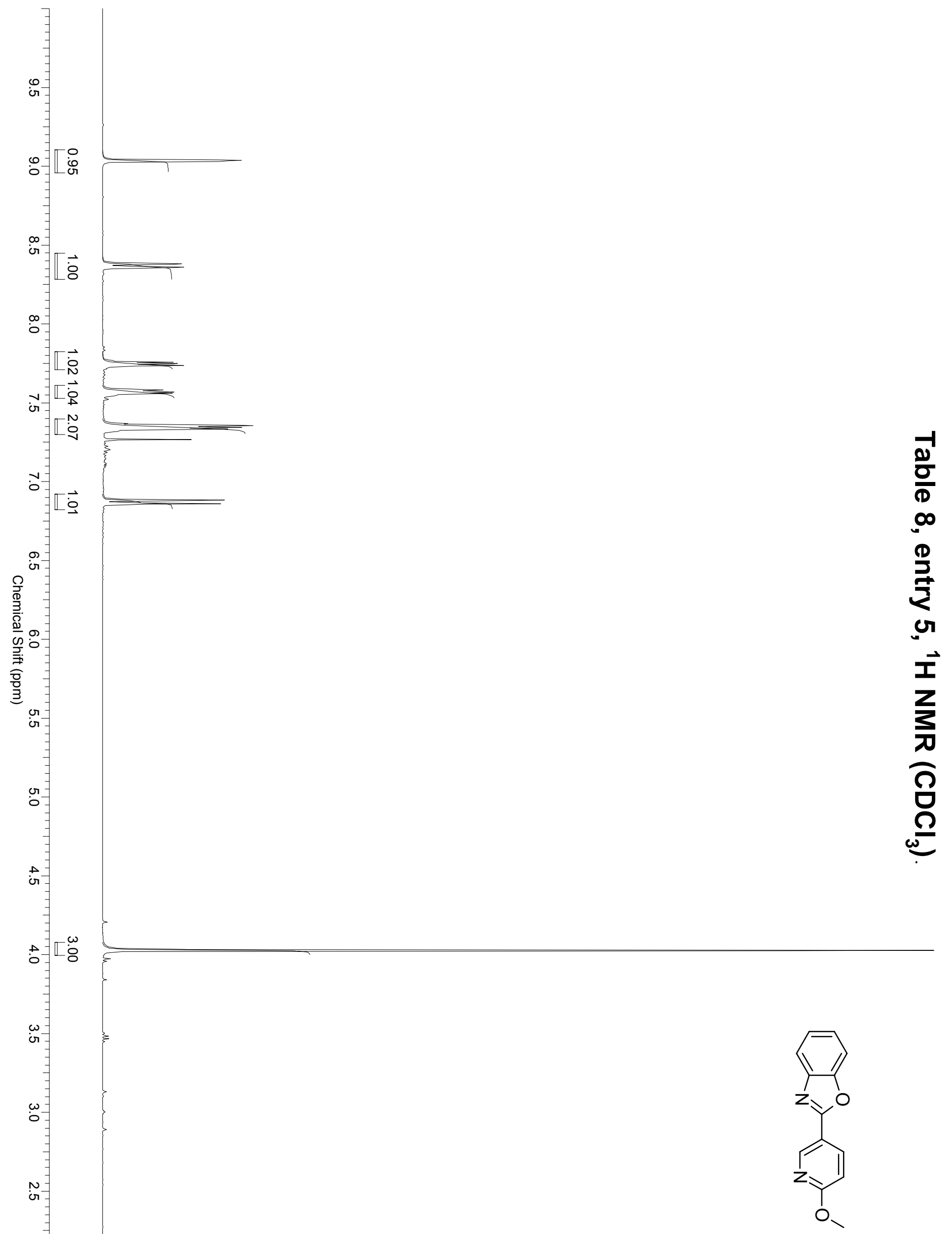




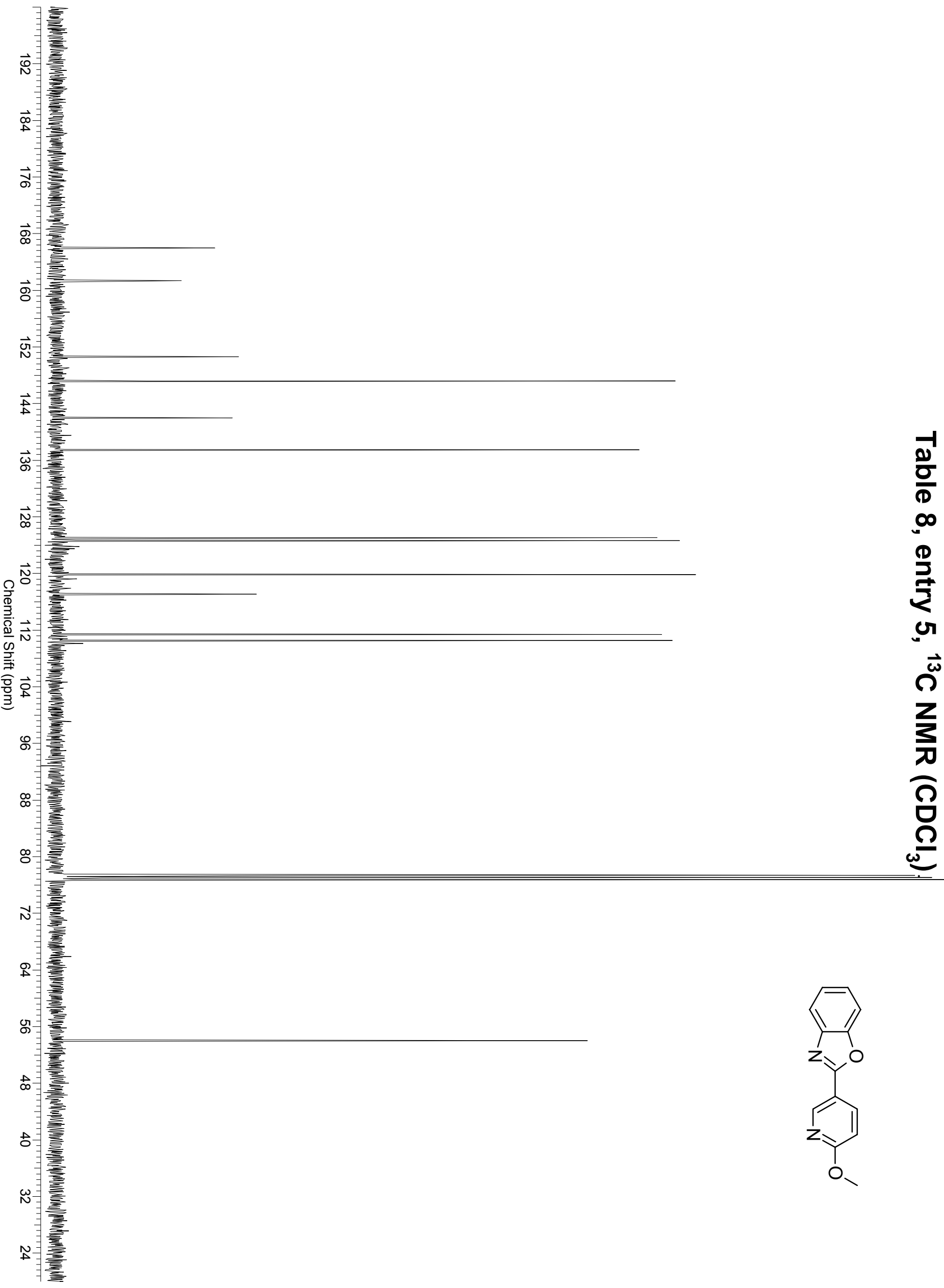




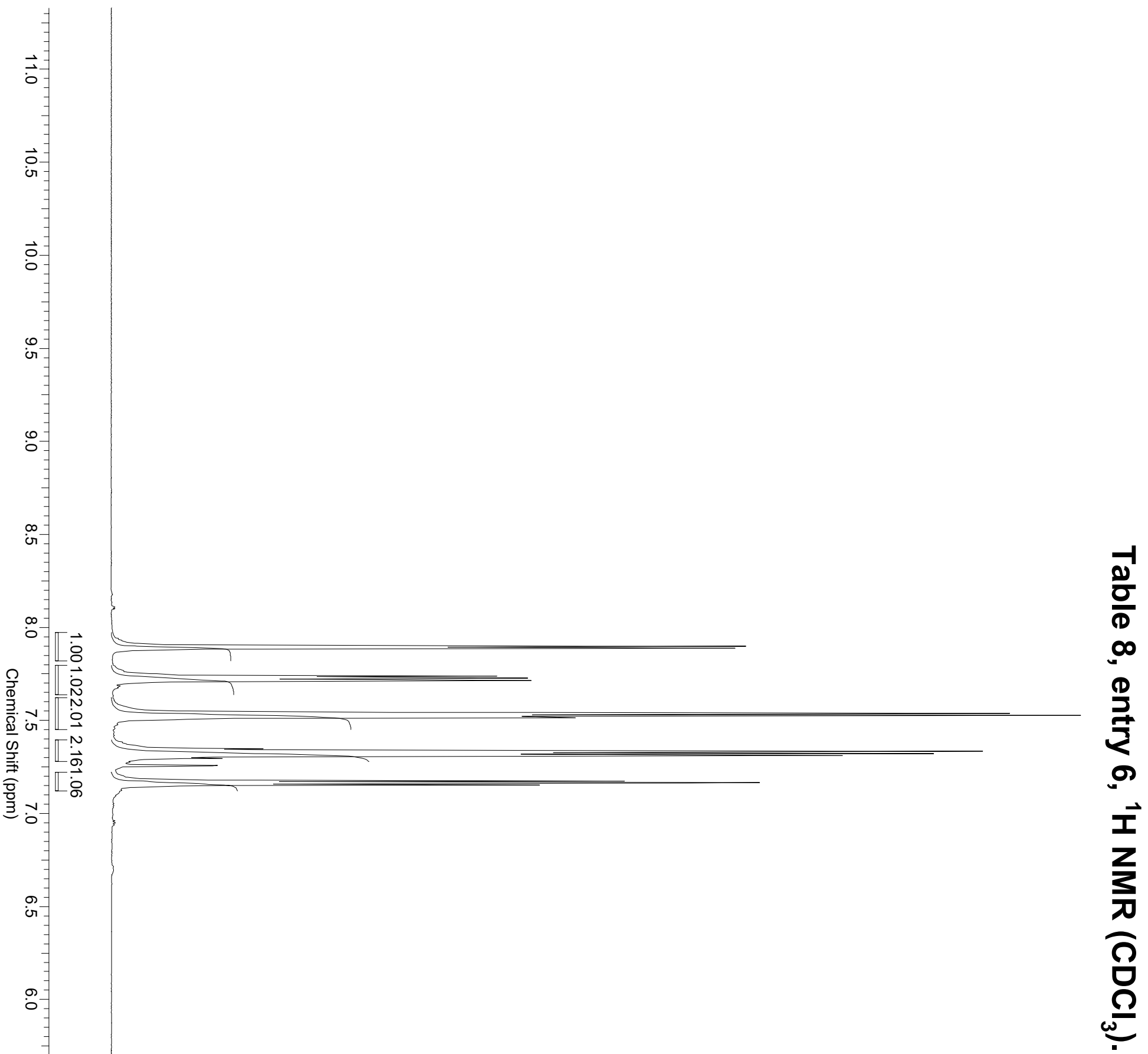

or
or
o
o
or

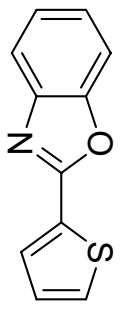




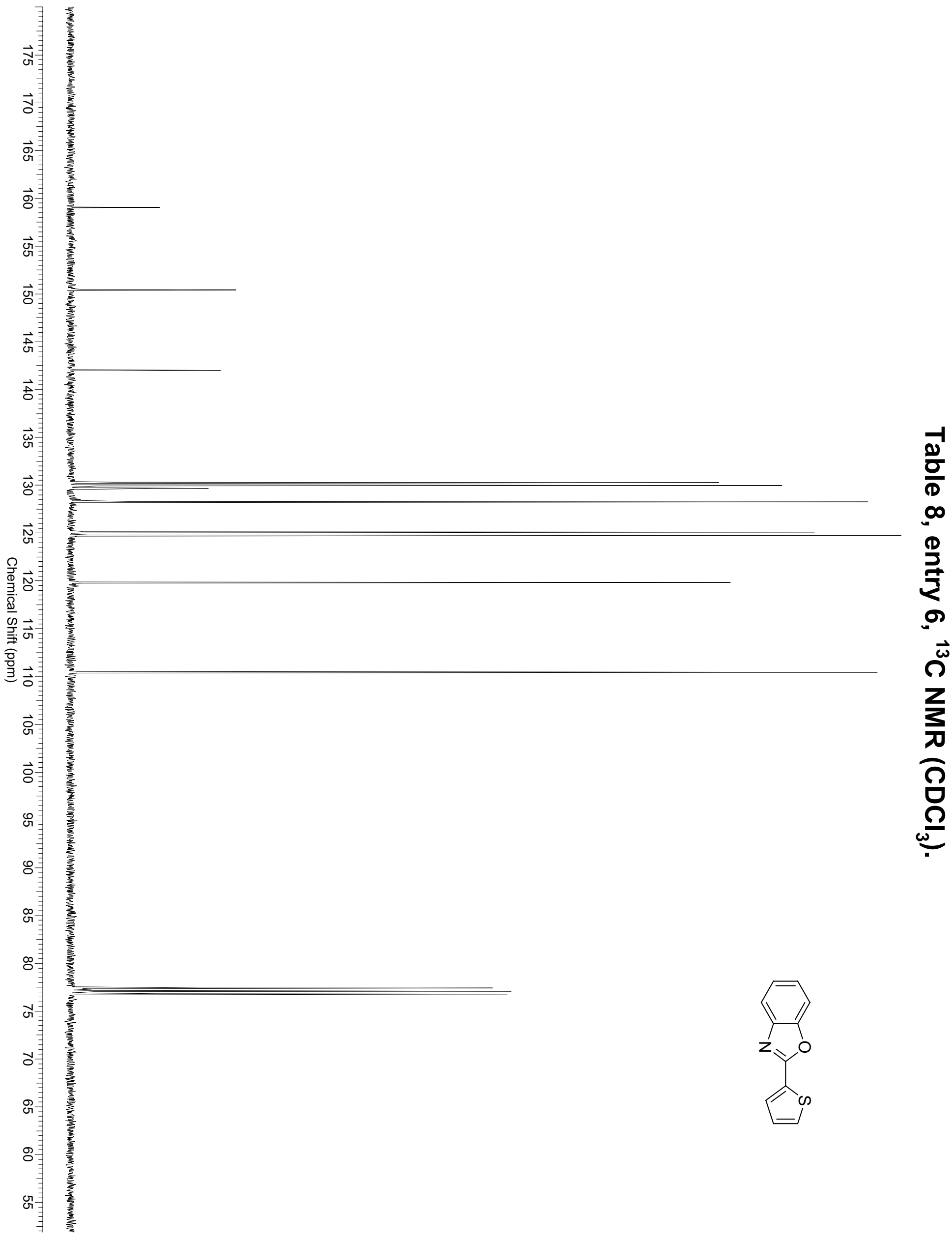




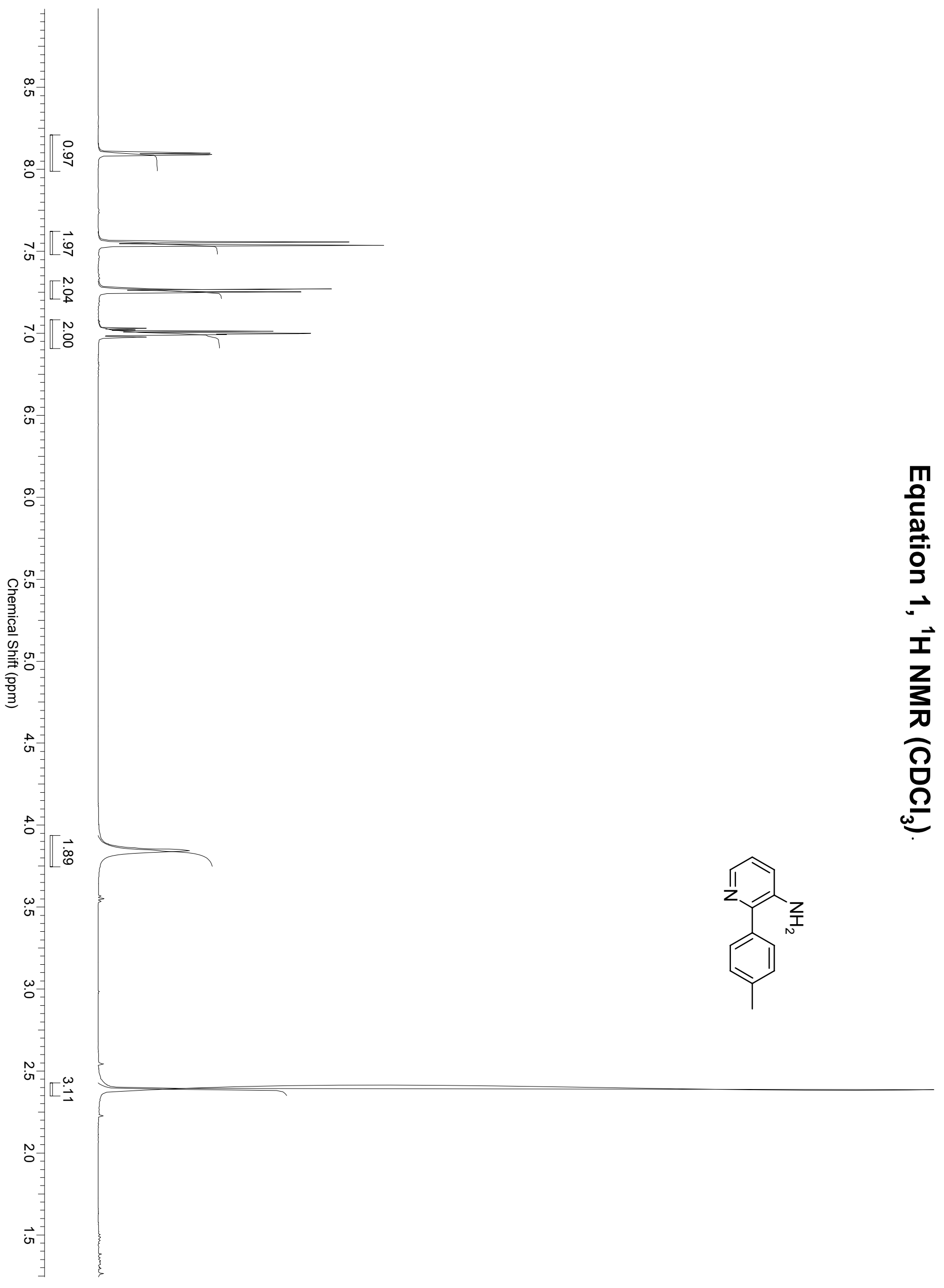




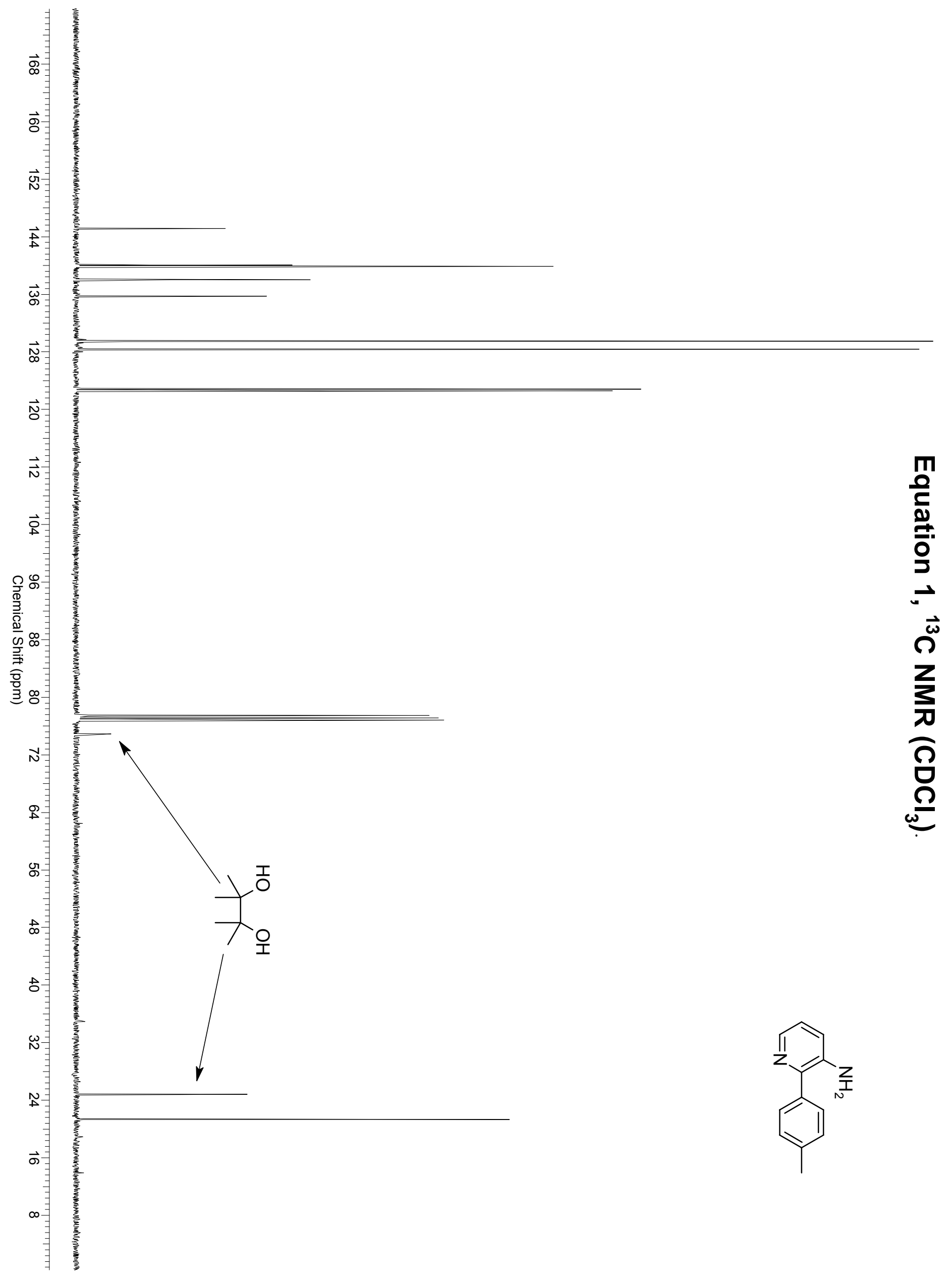



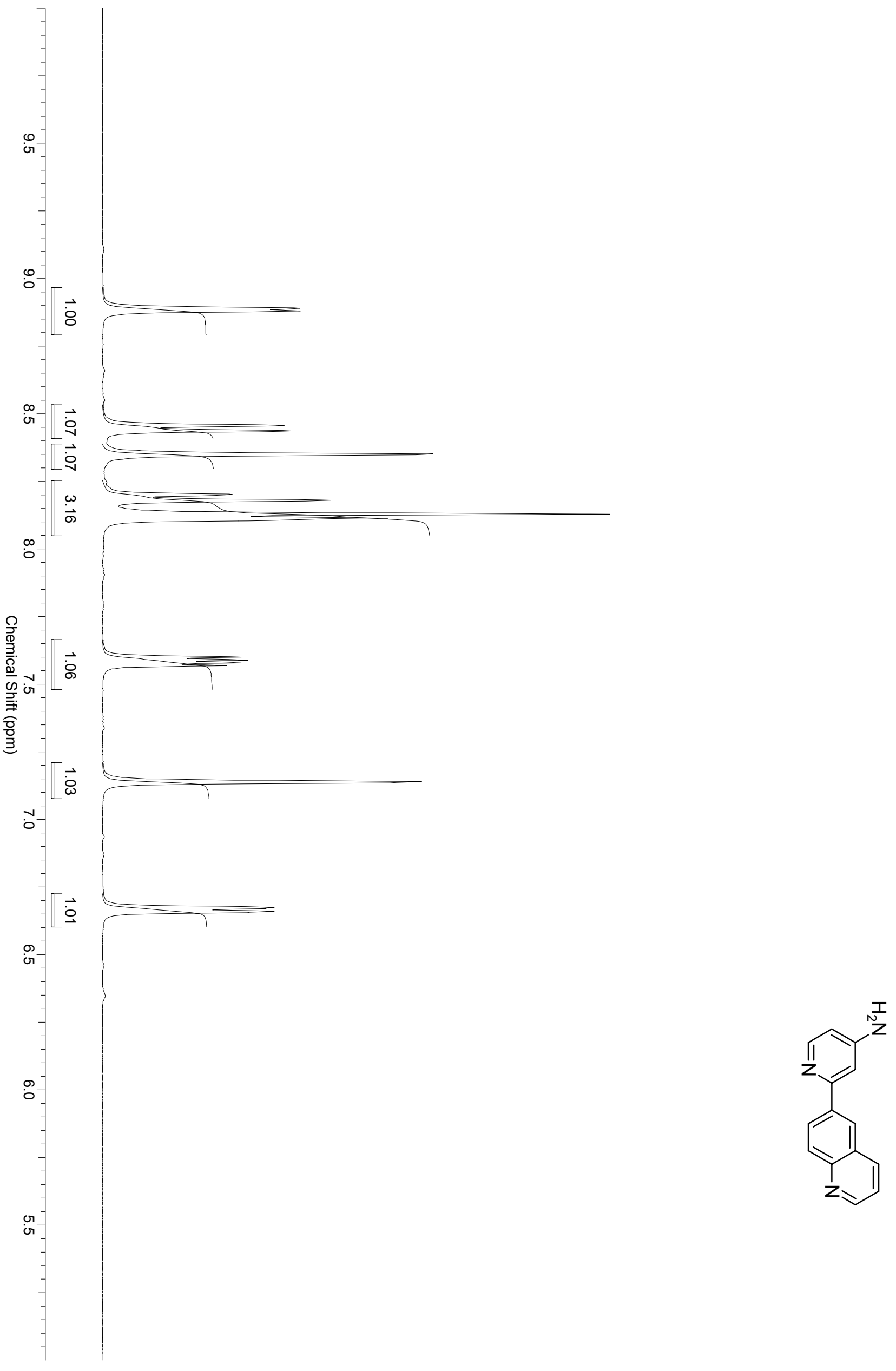


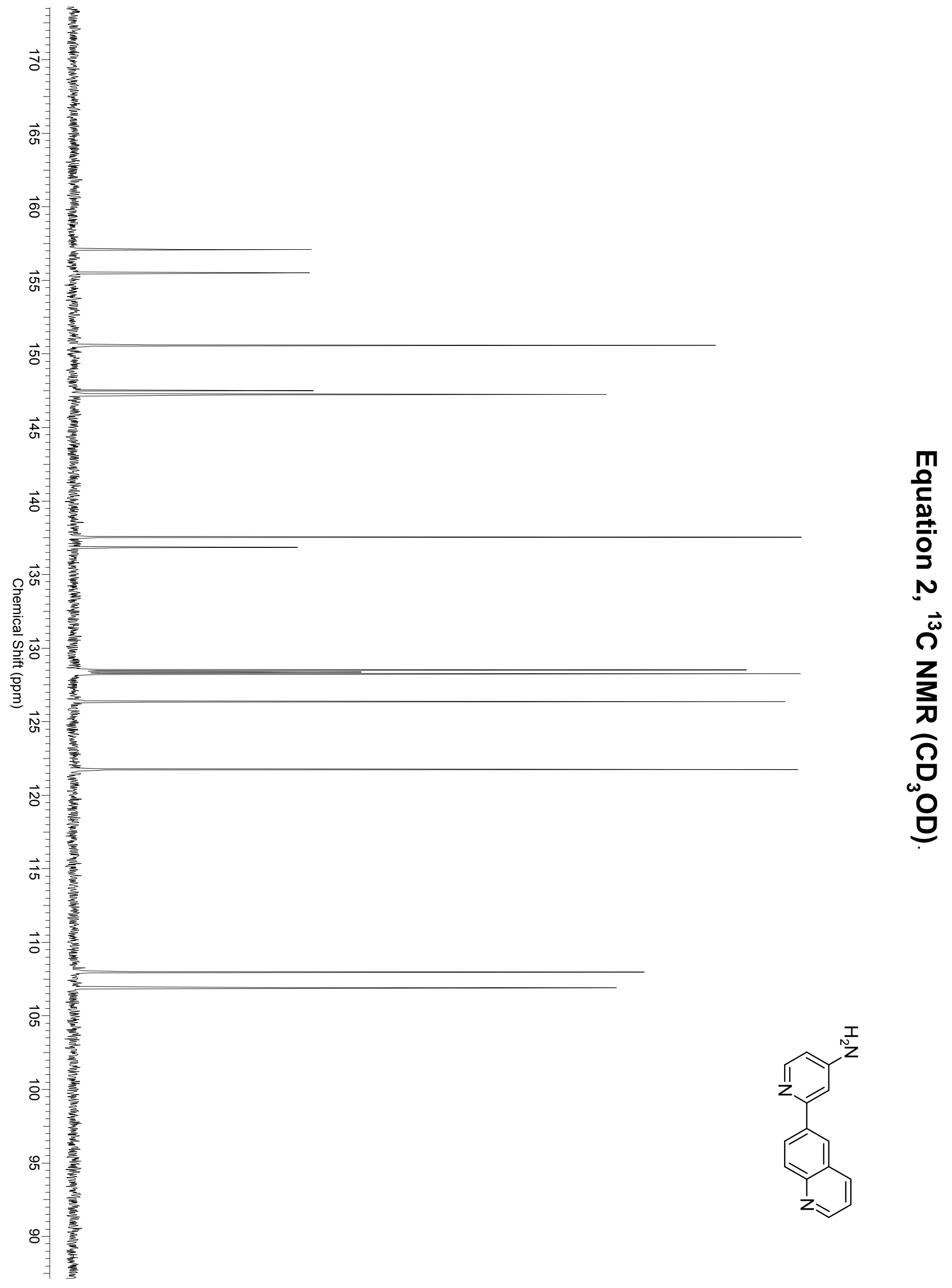

